\title{
International Petroleum
}

\section{Statistics Report}

February 1998
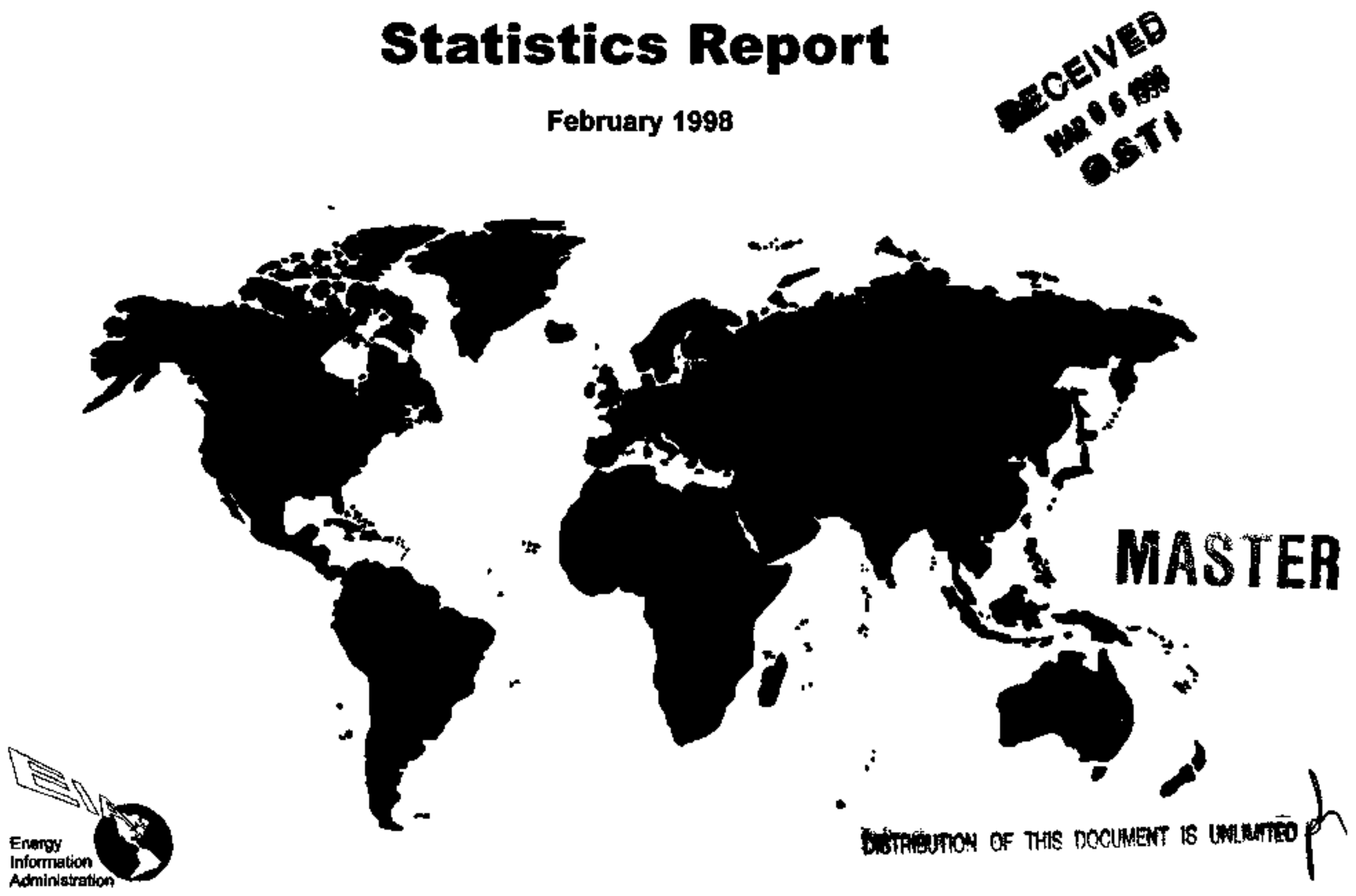


\section{HOW TO OBTAIN EIA PRODUCTS AND SERVICES}

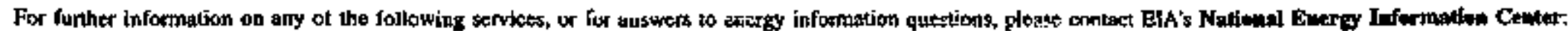

Natioual Energy Information Center (NEIC) (202) $986-8800$

Energy Infomlition Adminisiration (202) 586-0727 (fax)

Fortestal Butlding Room IF-048 TTY: (292) 586-1 I8I

Washington, DC 20585

\section{Electronic Proutacts ind Services}

ElA's Internet Sile Serves ofte nearly all ElA publications, Users can view and download selected pages or entire reports, search for information, download EiA dala and atalysis applications, and find oul aboun new EIA information products and servicts.

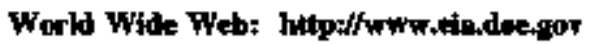
Gepher: gophert/gophericiaderegor
FIP; npolttp.eiondoejgiv

EIA glso dffers a listserve service for ELA press reveases and odher shon documents. Sign up on the ELA World Wide Web site.

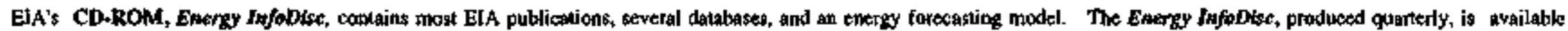
for a fec from STAT-USA, Dęartureme of Commence, 1-800-STAT-USA.

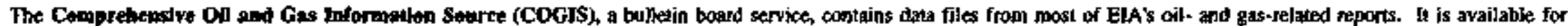
a fee from STAT-USA, of I-800-STAT-USA.

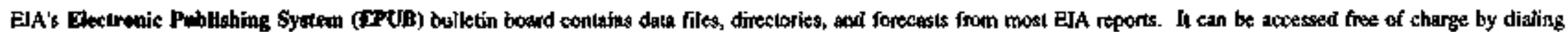
(202) $586-2557$.

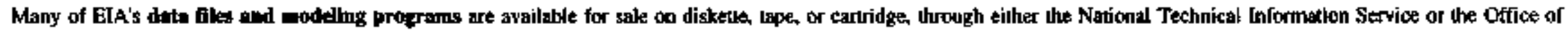
Scientific and Tecturical lefonmation, Departenent of Energy. Contact NEIC for information on specific products, sources, and media, and ordering itstruclions.

\section{Printed Poblications}

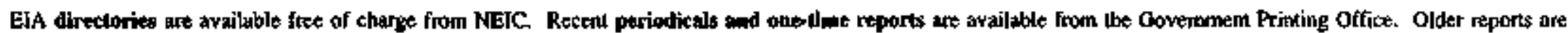
available from the National Technict lnformation Service:
Seperintendant at Docwemets
National Textmical Infonation Sertice
U.S. Govermmem Primting Office
U.S. Department of Commese
P.O. Box 37195
Plesturyth, PA 15250-7954
Springtielt, VA $2216 \mathrm{l}$
5285 Port Royal Road
(202) 512-180t: (202)-5t2-2250 (fax)
(703) 487-4650, (703) 32[-8547 (fax)

Peieased for Printing: February 23, 1998

Questions concerning the contents of this report should be directed to Michael J. Grillot, (202) 586-6577. 


\section{DISCLAIMER}

This report was prepared as an acogunt of work spossored by an agency of the United States Govermment Neither the United Stutes Gowernment apo any agascy thered, nor any of their emplayees, mistes any warrinty, express or inpliect, or assumes asy legal liability or responsibility for the accuracy, sompletepess, of use-

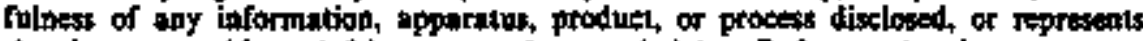
that its use would not infringe prrately owned rights. Refereace berein to uny spe. vific oommerciol prodwet, process, or service by trade name, trademark, manvfactuter, or otherwise does not pecessarily constitute or imply its endorsement, recommendation. or favorias by the Unlted Stales Gevernment of any agency therwof. The views aed opinions of authors expressed hercis do not bectostatily state or reflect those of the United Staves Goverament or any agency tiereot. 


\section{DISCLAIMER}

Portions of this document may be illegible electronic image products. Images are produced from the best available original document. 
DOE/ELA.0520(9802)

Distribution Category UC-950

\title{
International Petroleum Statistics Report
}

\author{
February 1998
}

Energy Information Administration

Office of Energy Markets and End Use

U.S. Department of Energy

Washington, DC 201585

This report was prepared by the Entery informestinn Adninistation, the indepeadent stustitical and

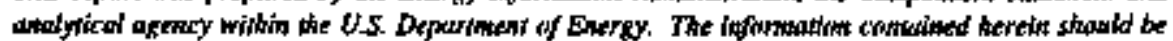

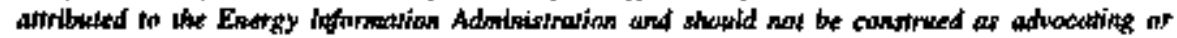

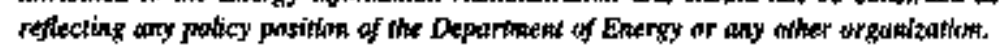




\section{Preface}

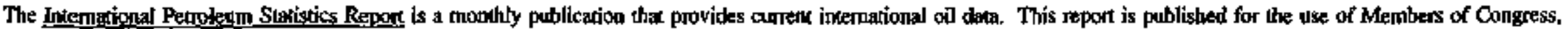
Federal agencies, State agencies, industry, and the general public. Problicadion of this report is in keeping with responsibilities given the Energy Information Administration in Public Law 95-91 (Section 205(a)(2)) that stgies:

The Aưuinistrator shall be responsible for carrying out a central, comprehensive, and unified energy data and information progran which will collect, evaluzie, assemble, analyze and disseminate data and information ..."

The Intemational Petrolenm Stalisstics Report presents data on international pol production, demand, imports, and stocks. The report has four sections. Section I contains time

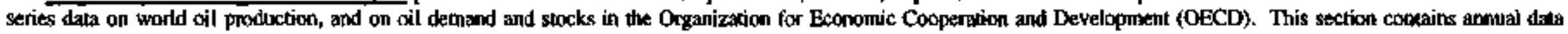
beginming in 1985, and monthly data for the most recent two years. Section 2 presents an oil supply/demand balance for the world. This balance is presented in quarterty inlervals for the most recent two years. Section 3 presents data on gil imports by OECD countries. This section contains annual data for the most recent year, quarterly dala for the mosi recem two quarters, and monthly data for the most recent twelve months. Section 4 presents annual time series daca on world oit production and oit stocks, dernsand, and trade in OECD countries. Wortd oil production and OECD demand data are tor the years 1970 throegth 199; OECD stocks from 1973 through 1996; and OECD trade from 1986 through 1996.

Daka for the United States are developed by the Exergy Information Administration's (EIA) Office of Oil and Gas. Data for othet countries are derived largely from published sources, including International Energy Agency publications, the ElA International Energy Annual, and the trade press. (See sources after each secrion.) All data are reviewved by the Incemational Statistics Branch of ElA. All data have been converted to unils of measurement farriliar to the American pubtic. Definitions of oil production and consumgtion are consistent with other ElA publications.

General information about this publication may be obtained from W. Calvin Kilgore, Director, Office of Bnengy Markets and End Use, (202) 586-1617, and from Louis D. DeMony. Direstor, Integrated Energy Statistics Division, (202) 586-6557. Detaiked questions and comments concerning the contents of the International Petroleum Statistics Report moy be directed to Michael J. Grillot, Tean Leadex, Intemaxional Energy Stalistics Team, (202) 586-6577; Patricia A. Smith (202) 586-6925, 01 to H. Vicky McLaine (202) 586-9412; Oil Market Chronology - Douglas Macinyre (202) 586-1831. 


\section{O'l Market Chronology .. January 1998}

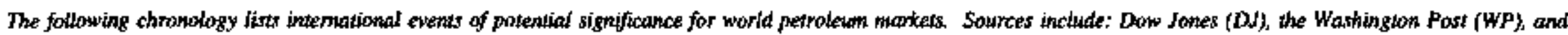
the Wall street foumal (WSI)

Jamuary 5 France's Tonal SA teports that it has successfully lested a new well in Black 14 offshore Cabinda, Angola The new discovery, called Landara, prodices 7,300 barrels per day of 36-degrac API conde ofit, encononging Total and its parthers to contimue with thre more exploralion wells in 1998. Total holds 20 percent of Block 14, with Chevron Corporation bolting 31 percent, Angola's national oil company Sontagol 20 percent, Italy's Agip 20 percent, ant Petrogal 9 percenk. (DJ)

January 7 Dae to the contiming Asian economic crisis, South Korea's refiness have reportedly cul operations 10 around 80 percent of capacity. The refiners have also had difficulty securing enude oll supplites for delivery in late January or February, which coutd cut operations to as low as 70 percent-75 percent of capacity. (DJ)

Impunry 8 Elf Exploration Angola, a unit of France's Etf Aquilaine, and its parnes Socieded Nacional de Combustiveis de Angola begin production of 9,500 barrels of oil per day from the Donbo field, localed oftstore Angola. Elf Exploration operats the field and holds a 50 percent interest. (DJ)

January 9 Premier Oil PLC announces that it has made a significane gas discovery in the Naluna Sea, Block A, Indonesia. After four drill tests produced a combined Ilow rate of 43 million cuhic feet of gas per day. Premier has suspended the Pelikan-l as a future producer. The Pejikan-1 well is locaked about 31 miles from the Anon oil production platom, and it folkws a gas discowery an the Gajah Puteri well drilted in late 1997. The oil rig will now move to another kocution in the block to trill the Adik Anoa dil and gas prospect. Premier operates Block A, bolding a 66.67 percent interest, with Kuwail Foneign Exploration Company holdting the remianing 33.33 persent interes. (DJ)

January 9 Shell Ptilippines Exploration, a unit of Royal DutchShelt and its partner Oscidental Philippints, a unit of Occidental Petroleum Corporation, sign two gas supply agreements for the Malampaya nabural gas-lo-power project in the Philippines. The furst agreenemt is with First Gas Hokings, a company 40 percent-owned by British Gas. The agreement calls for L,46 Itillion cubic feet of natural gas lo be sent over a 20-yedr period to two power plants that will be buit by First Gas. Initial delivery is scheduled to begin Jarwary 1,2002 . The second agreemem is with National Powwe Corporition of the

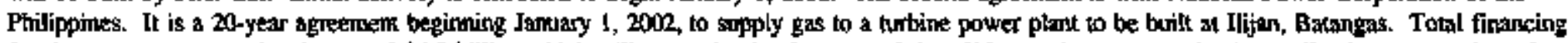
for the gas-to-power project is around $\$ 4.5$ billion which will cover the development of the offshose subsea gas production wells, dhe consiroction of a 317-mile offshose pipeline and sthere terminal, and the construction of the three power plants. (DJ)

Jamuary 12 The Albanian governmenk awarts oil explocation blocks to two intemational oil consortia, one led by Austria's OMY and the orher led by an Albanian subsidiary of Oochderkal Petroleum. OMV and jis United Kingdom partners Enterprise Oil and Clyate Gas have received two blocks in the soutt and one in central north Albania. Meanwlyike, Occidenas and jis partnes International Petroledm Corporation have receivod two blocks in the south and one in the noth. Although the production-sharing agreements still need to be approved by the Albanian govermment, drilling is expected to begin in mid-1998. (D))

Janumry 12 After running aground in rough seas near the United Arab Eminzes on January 7, 1998, a sunken targe has spilled about 28,000 barrels of fuel oil into the Perstan Gulf, . The spili crealed a 5 mile by 2 mile oil slick along the coestline of the northem emirate of Umm Al Quwain. Intemational envirommental experts have teamed with the Emirates Coast Guand, the Federal Environumeon Authority and the Abu Dhatji Nation:sl Oil Company in cleamup efforts. (D)

January 13 Japan's Mitsubishi Peiroleum Developanemt Company begins oil prodiction from a wetl in Block 3\$1, located 25 miles off tho coast of Angola Daily output from the wetl is estimated at 9,500 barrets, with the concession expected to yield a total of around 15 million barrels of crude oil. (DJ)) 
Jameary 14 In a show of suppost for Richswd Butke, chaimman of the United Nations (U.N.) Special Commiasion on lraq, the U.N. Security Council unanimously approves a satkement deploing Iraq's recent actions to impede inspections by U.N. weapons moritors. (D)

China Netional Offshore Oit Corporation and Norway's Statoil begin production from the Lufeng 22-1 ofl field. Lufeng 22-1 is the deepest oil field in the South China Sea and is curnently profucing about 60,000 barrels per day. (D)

Irag begins exporting curde okl under the third phase of the United Notions sponsered oll-for-food program. First loadtugs are ftling the mbltion bartet

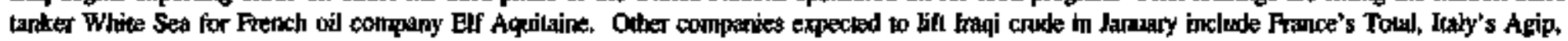

Russia's Lukoil, and Ching's Sincotern, (DJ)

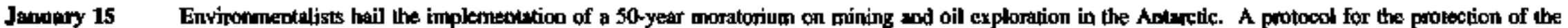
Antartic was axlopted by twenty-six countries in 1991, but it could not be implemented until Sopant's ratification clearad the way last month. Antarctics contsins 70 percent of the word's ftesh walter, and the moralorium alternpts to preserve the wordd's least poilhnted contionnt. (WP)

Junuary 19 ElI Exploration Incorporated, a subsidiany of France's Elf Aquitaine announces the development of its Vhrgo discovery locsted in the Gulf of Mexico of the coast of Louisianis. The next step for Elf and its partners is to consunct a drilling and poduction platfortin thal will have a datly productlon capacily of 120 million cubic feet of gas and 15,000 banrels of cil. Elf Exploration holds 264 percent ibterest in the ficense with Coastal Oil and Gas 16.2 percenth, Pogo Producing Compariy 10.8 percent, and Nippon Oil Exploratbon USA Límited 9 petcent. (D)

Bohad Petroteum Company, a unit of China National Orfshore Oil Corporation, begins production from the Boxi Oil fiekd kcated in the Bothai Sea. The field's reserves ane estinzaled at more than 37 million barrets of oil and abouk 353 billion cubic feet of natural gas. (DJ)

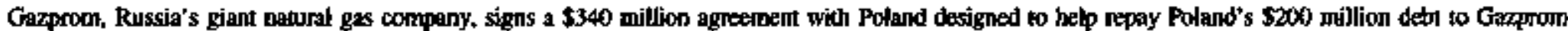
and io finance the Jamaj-Exropa gas pipetine. Unika the agreement, Poland will glve Gazprom $\$ 175$ millioe worth of goods and services. The ofker $\$ 165$ million will fimance the conssourtion of the pipetine, wfich will cross Russia and Eastem Europe imlo Westem Europe. The Polish section of the pipeline is expected to be completed later this year. (DJ)

Junnary 20 Russia's second and seventh largest oil concens, AO Yukos and AO Sibnef, annonnce a merger to creale a new company to be named AO Yuksi. In terms of neserves, Yuksi will surpass Royal Dutch/Shell Group as the world's largest private oil conpany and mink third behind Shell and Exxon Corporation in terns of production. Yukos's stareholders will take a 60 percent share in the new company, with dxarekolders from Sibmeft controlling the remaining 40 pencent. (WS))

January 21 Following a speech last week by Luis Giusti, presideon of state-owned Peiroleos de Venezuela, in which he claimed dum Sandi Arabia has been producing 9.12 millian barrets of enule gif per day and that its overpochetion has led to falting oil prices, Sauti Arabia defends itself stating that it is abiding by its new OPEC production quots of 8.76 milition banels per day. Giusti also said thec Venezrela is currently producing 3.3 million banrels per day, which

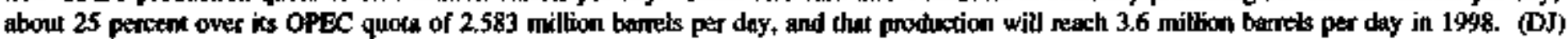

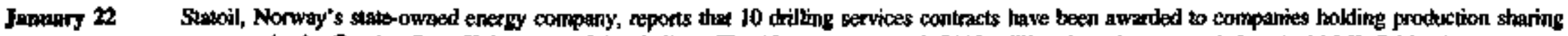
contsacts in the Caspian Sea of the coast of Azerbaijat. The 10 contracts, worth $\$ 110$ million, have been awanted to Azdri MI, Schlumberger, Geoservices, Oceaneering, Baker Hughtes lntec, Franks Internesional and Security DBS. The first explortion well in the Shah Derulz area, where Stanjil lolds a 25,5 percent interest, is scheduled to be difled in hune 1998. (D) 


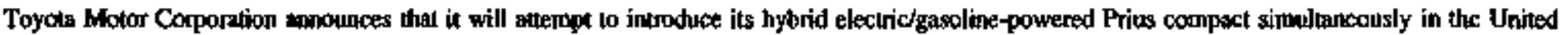
States and Europe in the year $\mathbf{2 0 0 0}$. This new bybrid system generates half the carbon dioxide of a gasoline engine and reduces enissions of other tonic solbstances soch is nitrogen oxides. The Prius was introduced in Japan in Decernber 1997, and sales have been strong, with 3,500 onders receiwed in the finst moath (DJ)

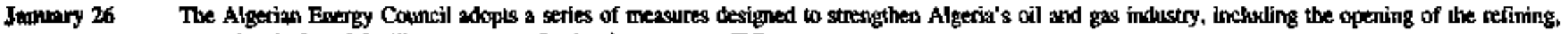
petroxhenical, and fertilizer gectors to Joreign investment. (DI)

Delegates fom OPEC's Ministerial Moxitoring Committe tokl an emergency one-day meeting to discuss falling oil prices. The delegates apree that OPEC's crude oil ouxput is above the 275 million bands per day ceiling by at least $\$ 00,000$ barrels per day, bux less than 1 million barrels per day, The delegales also say that duene is litile OPEC can do to lift oil prices soon outside of Jecomrmending strict adherence to production quotas or possibly curting production. (D])

Janumry 27 Su Shutín, Execative Depucy Director of the Daqing Petroleum Managentent Bureau, stales lhat China's lagest oit field, Daqing, will maintain antual

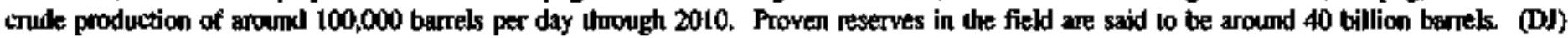

Jamiang 28 Ambco Corporation announces a crude oil discowery at its Innootelle oil field, located 34 miles off the east coast of Trimidad. This represents Amoco's largest crude oil find in Trinidad in 25 years. Reserve estimates range between 40 and 70 million barrels, and as many as eight wells could be drilled by the end of the year. [Editor's note: ELA estimates Trinidad oil production for 19y7 at 124,000 barres per day.] (DJ)

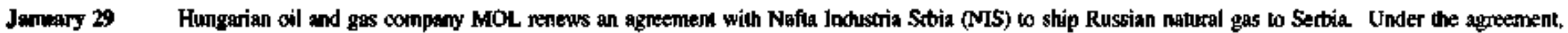
MOL will ship 99 billicin cubic feed of Russias gas across Hungary from the boider wilh Ukraine to the Hungarian-Serbian horder in I998. Anthial gas shipments to Sertia will gradually inctease over the course of the sgnement eventually reaching 134 billion ctibic teet in 2002 . Over the text 20 years, MOL expects to ship a total of at least 2.6 urllion cubtc feet of gas to Sextia. In addition, MOL is ser to develop a pipeline system to iteliver naural gas to Bosnia. MOL says it will invest aurond $\$ 77$ million in the new syolem and expects to have if in servec by (he year 2000 . (DJ)

Jamary 31 New York, Massachusetts, Vemont and Maine have decided to hold oat for Califomia's zero emission standard for cars rather than join the federal National Low Emissica Vehicle peogram. Under the federal program, automakers will agieo to begin producing cars that will significantly rectuce the arnount of smog-producing emissions if all 13 northeastem stales will agree not to adopt Catitorma's tougher standard. The automakers say that the new cars would be 70 percent more effective in eliminating emissions of hydrocarbots and 50 percent more effeclive in reducing emissions of oxides of nitrogen. The cleaner cars could be avgilgble in the northest as sarly is this year and notionally by 2000 . Many federal ofticisls and sone autornakers believe thal the auto industry will agree to the new program even without parlicipatton from all 13 states. The autontakers have unil February 17 bo decide whether they will go aloog with the National Low Emisstion Veticle progeram. (WP) 



\section{Contents}

\section{Section 1. Annugl and Monthy Os Date}

Table 1.1a World Crude Ois Production (Inchuding Lease Condensone), 1985-Present $\ldots \ldots \ldots \ldots \ldots \ldots \ldots \ldots \ldots \ldots \ldots \ldots \ldots \ldots$

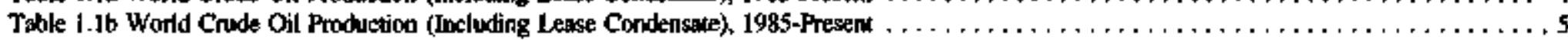

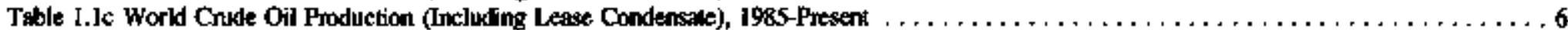

Tahle 1.2 OPEC Crude Oil Production (Excluding Condenspate $\}_{r} 1985-P$ resent $\ldots \ldots \ldots \ldots \ldots \ldots \ldots \ldots \ldots \ldots \ldots \ldots \ldots \ldots \ldots \ldots$

Table 1.3 World Natural Gas Plant jiquids Prochuction, 1985-Presen $\ldots \ldots \ldots \ldots \ldots \ldots \ldots \ldots \ldots \ldots \ldots \ldots \ldots \ldots \ldots \ldots \ldots \ldots \ldots$

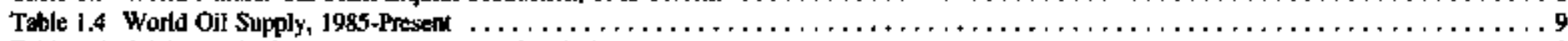

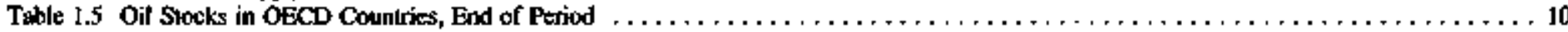

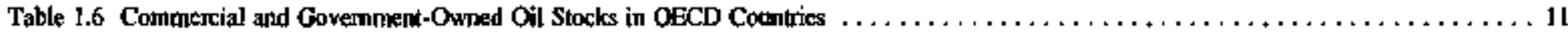

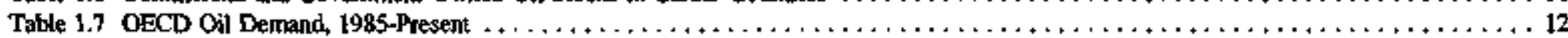

\section{Section 2. Intemationol Oa Balance}

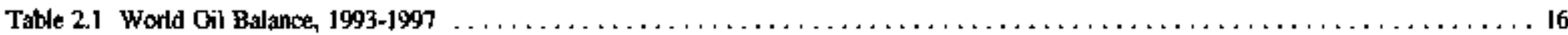

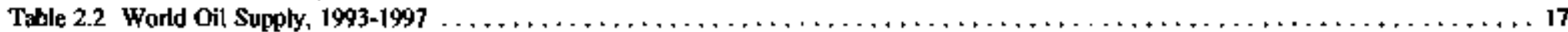

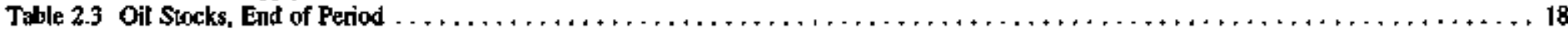

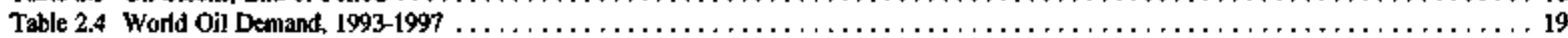

\section{Sextion 3. Entenorional Oil Imports}

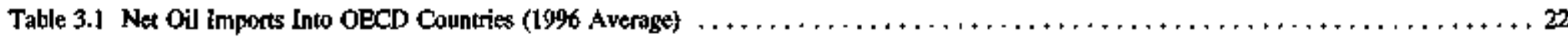

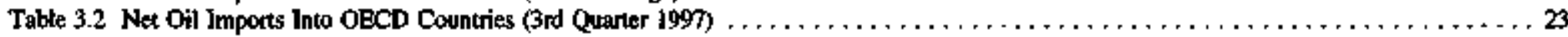

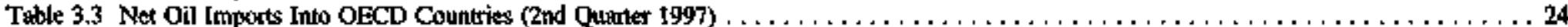

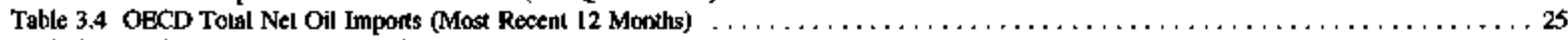

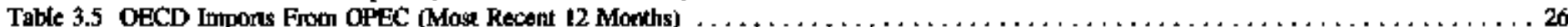

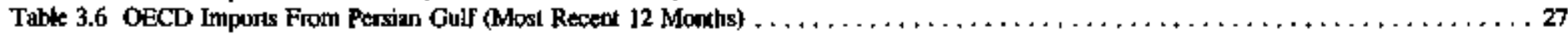

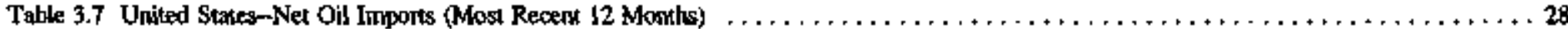

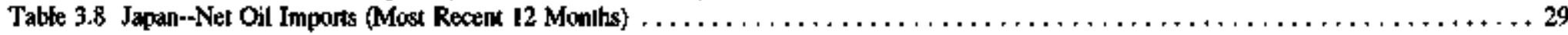

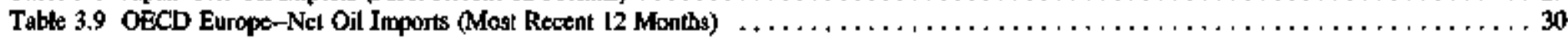

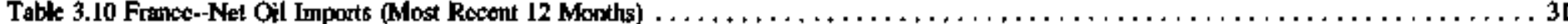

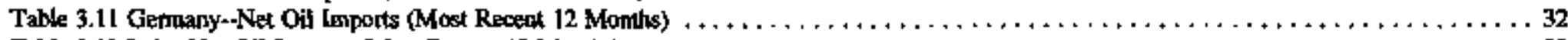

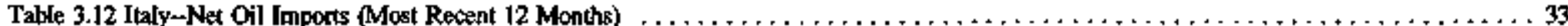

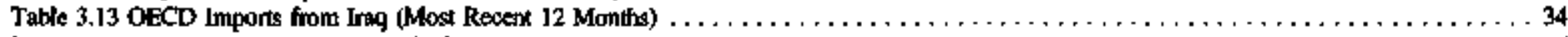

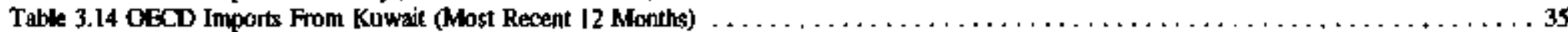




\section{Contents - Continued}

\section{Seetion 4. Hiatertical OA Doto Serles}

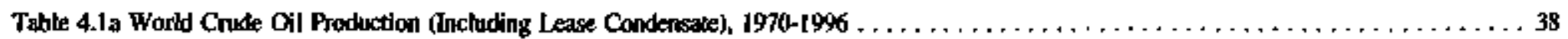

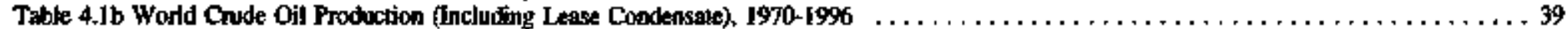

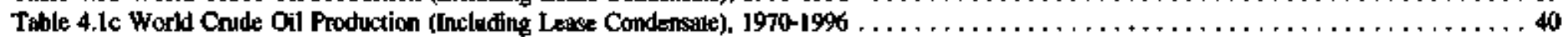

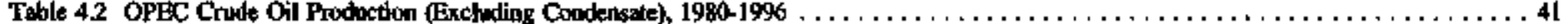

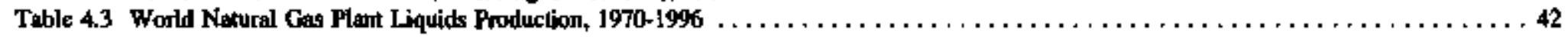

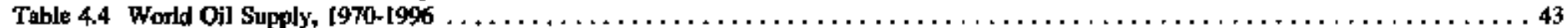

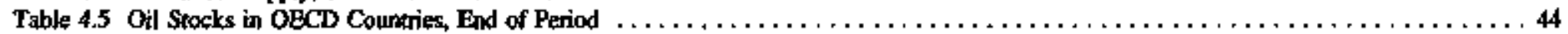

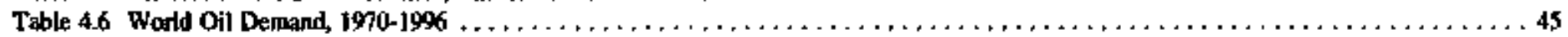

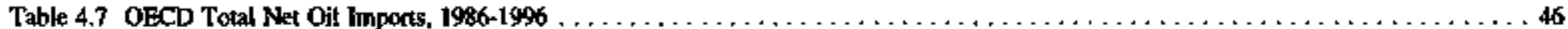

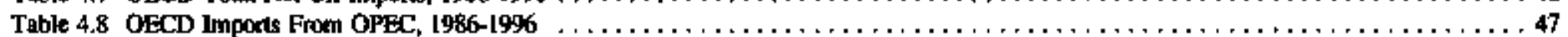

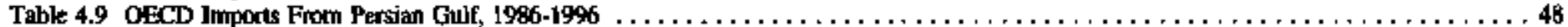

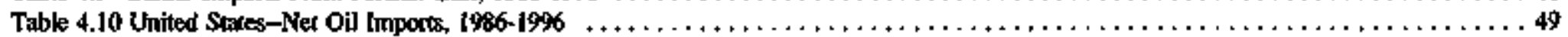

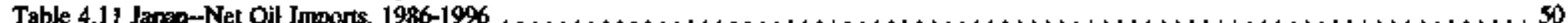

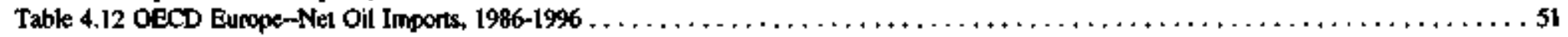

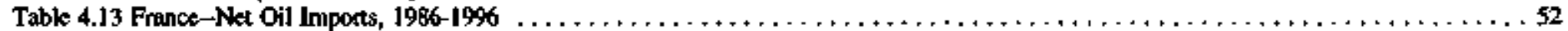

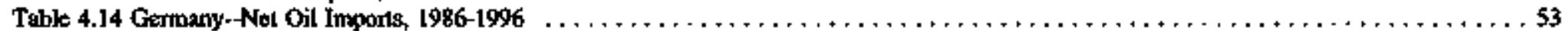

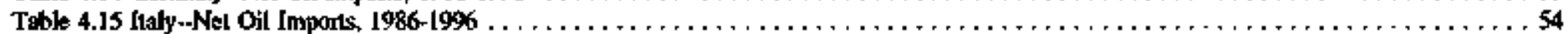

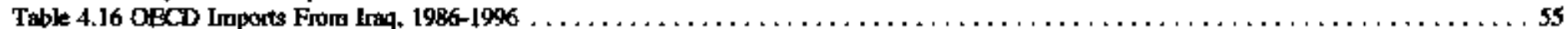

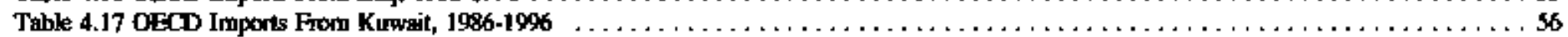

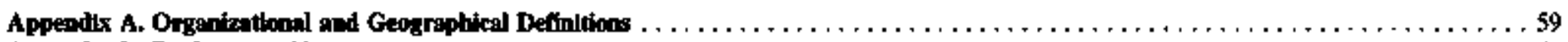

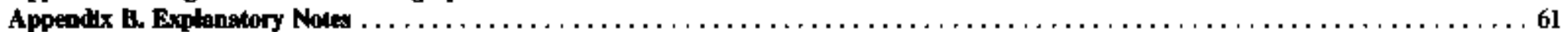

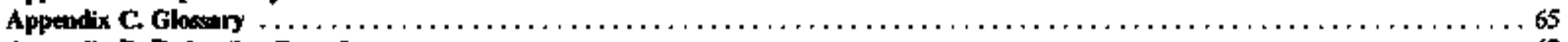

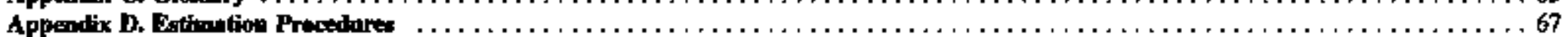

Apperodix E. Conversions

Table E1. Refined Petroleum Products Conversion Factors $\ldots \ldots \ldots \ldots \ldots \ldots \ldots \ldots \ldots \ldots \ldots \ldots \ldots \ldots \ldots \ldots \ldots \ldots \ldots \ldots \ldots \ldots \ldots, \ldots \ldots$

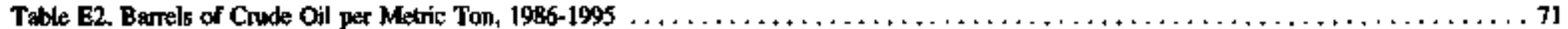

\section{Figarea}

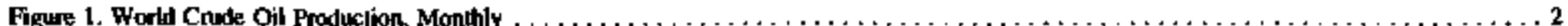

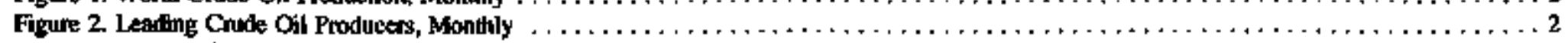

Figure 3. OECD oil Consumption, Montity

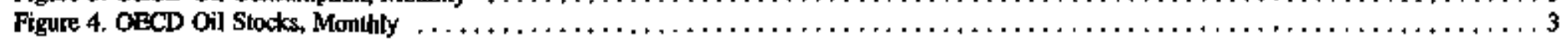


1. Annual and Monthly OII Data 


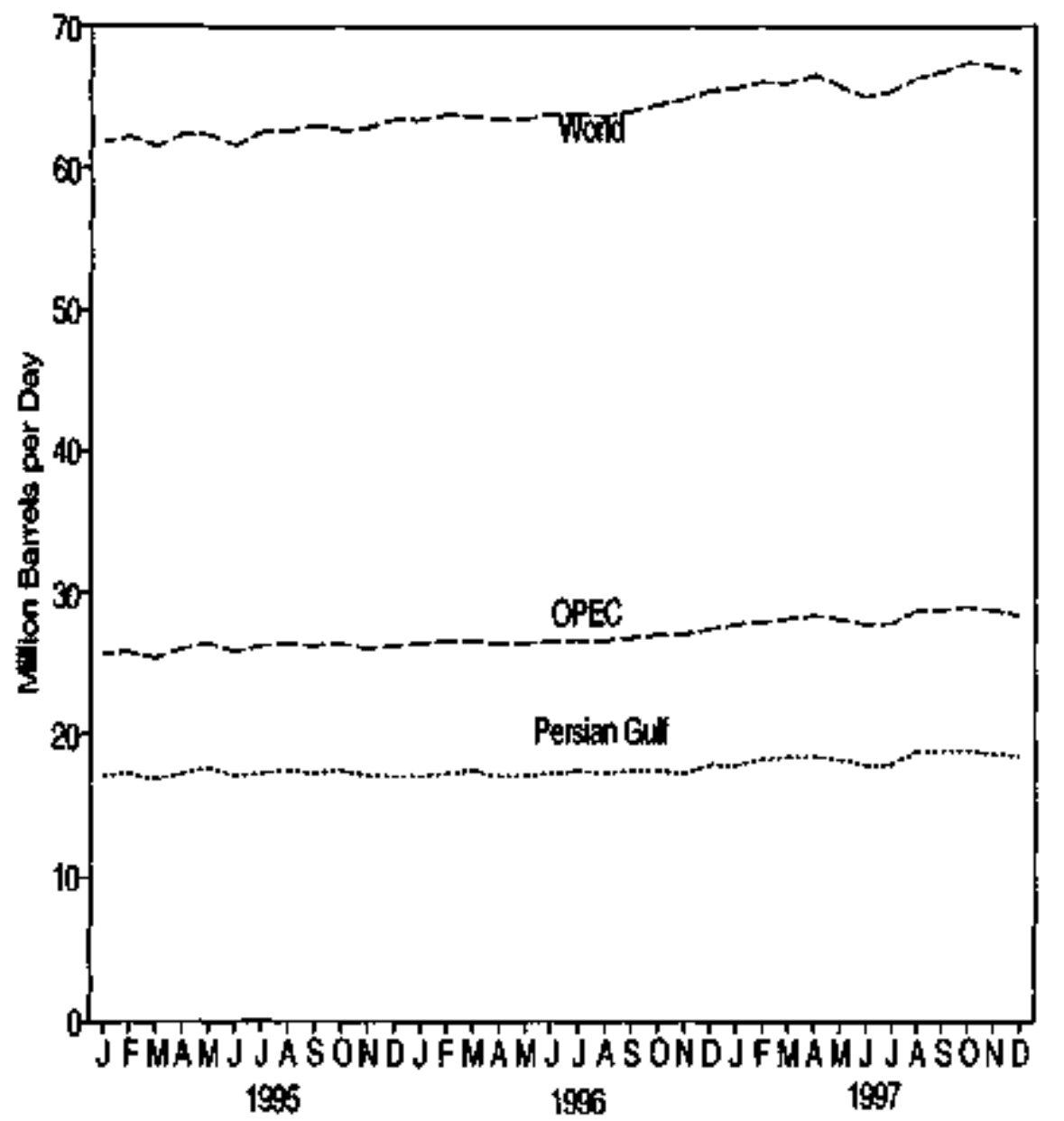

Source: Tables 1.1a-1.1c

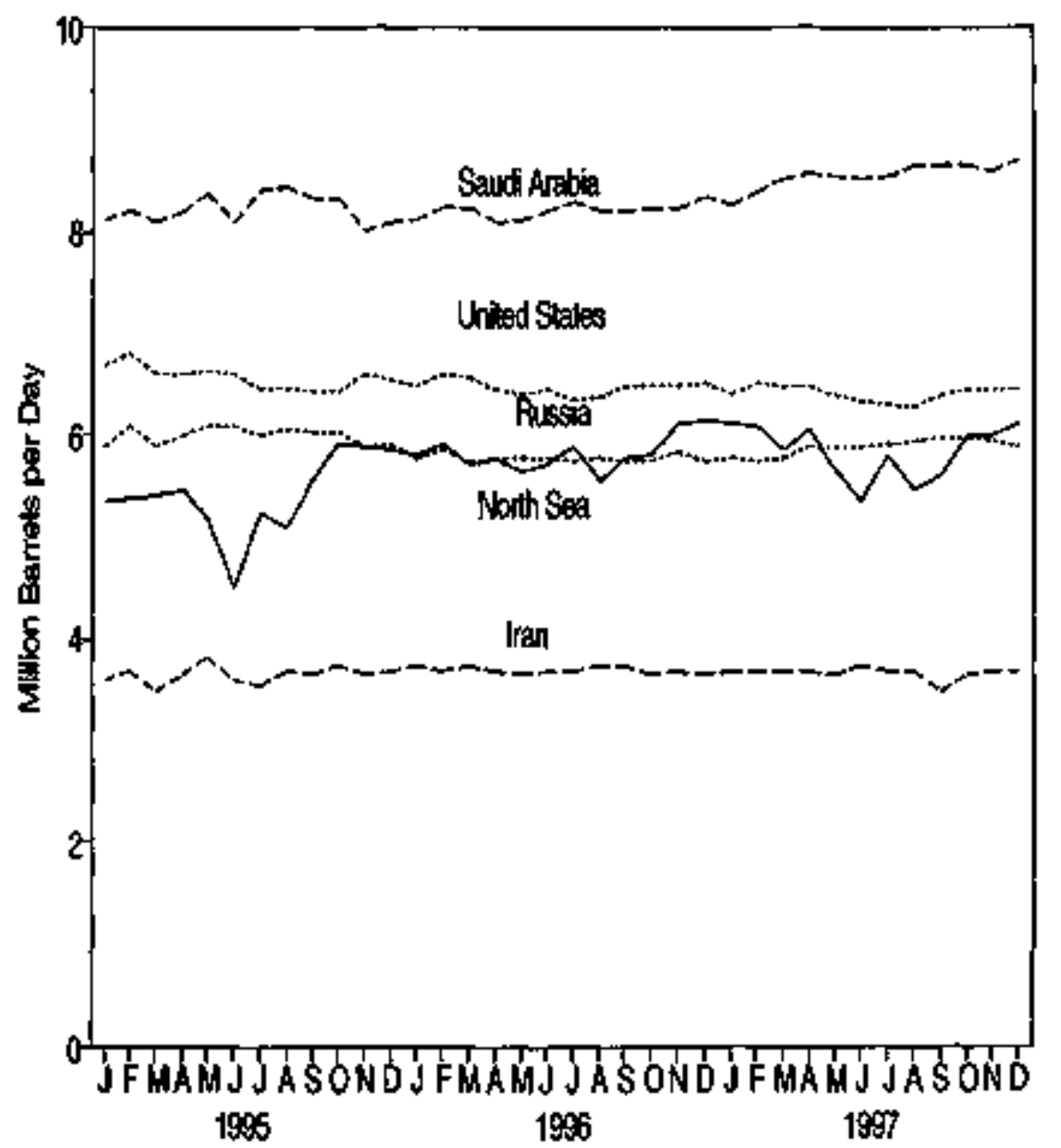

Source: Tables 1.1a-1.1e 
Figure 3. OLCD oil Consumption, Nonthly

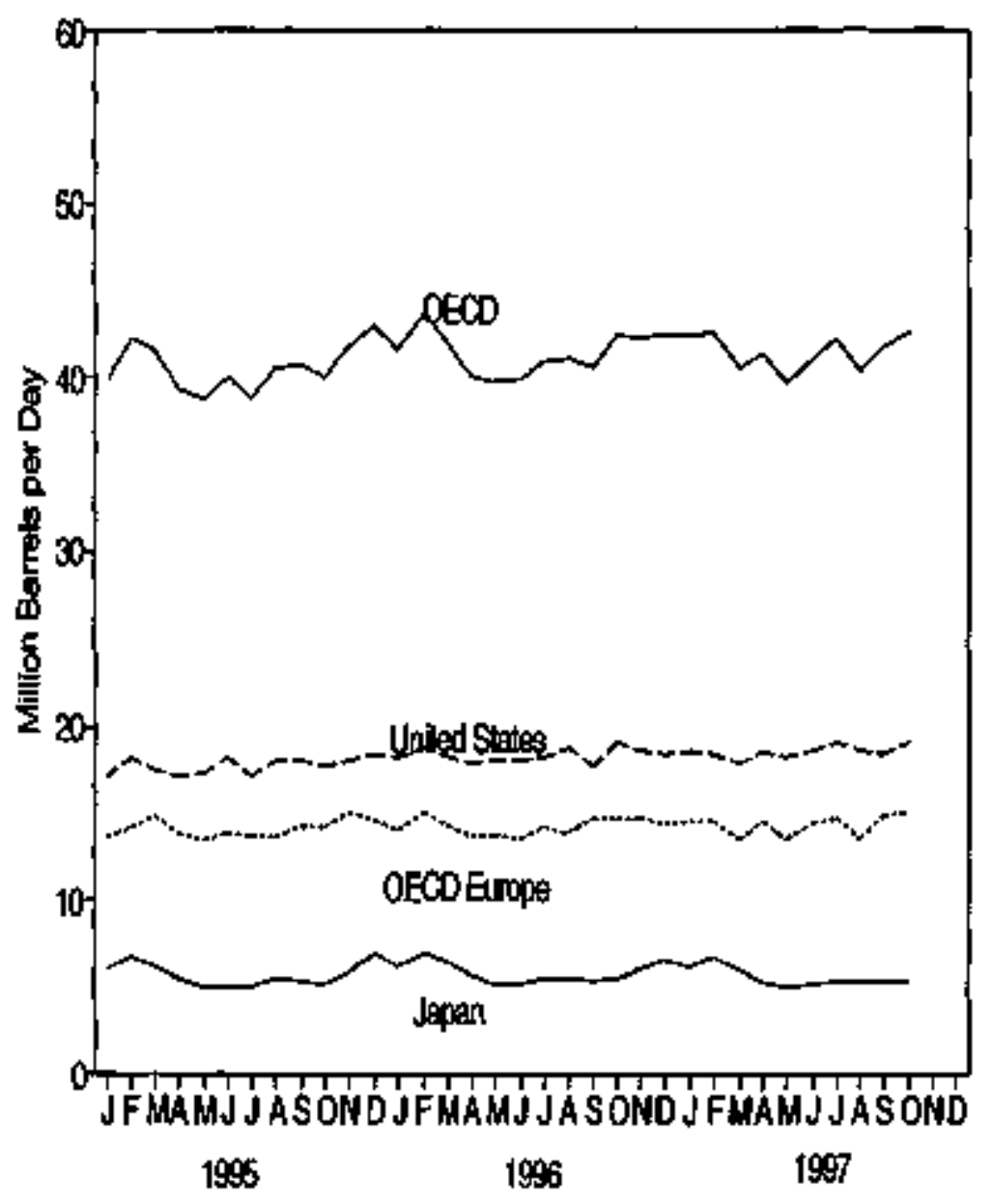

Source: Table 1.7
Figure 4. Osch oil stocks, wonthly

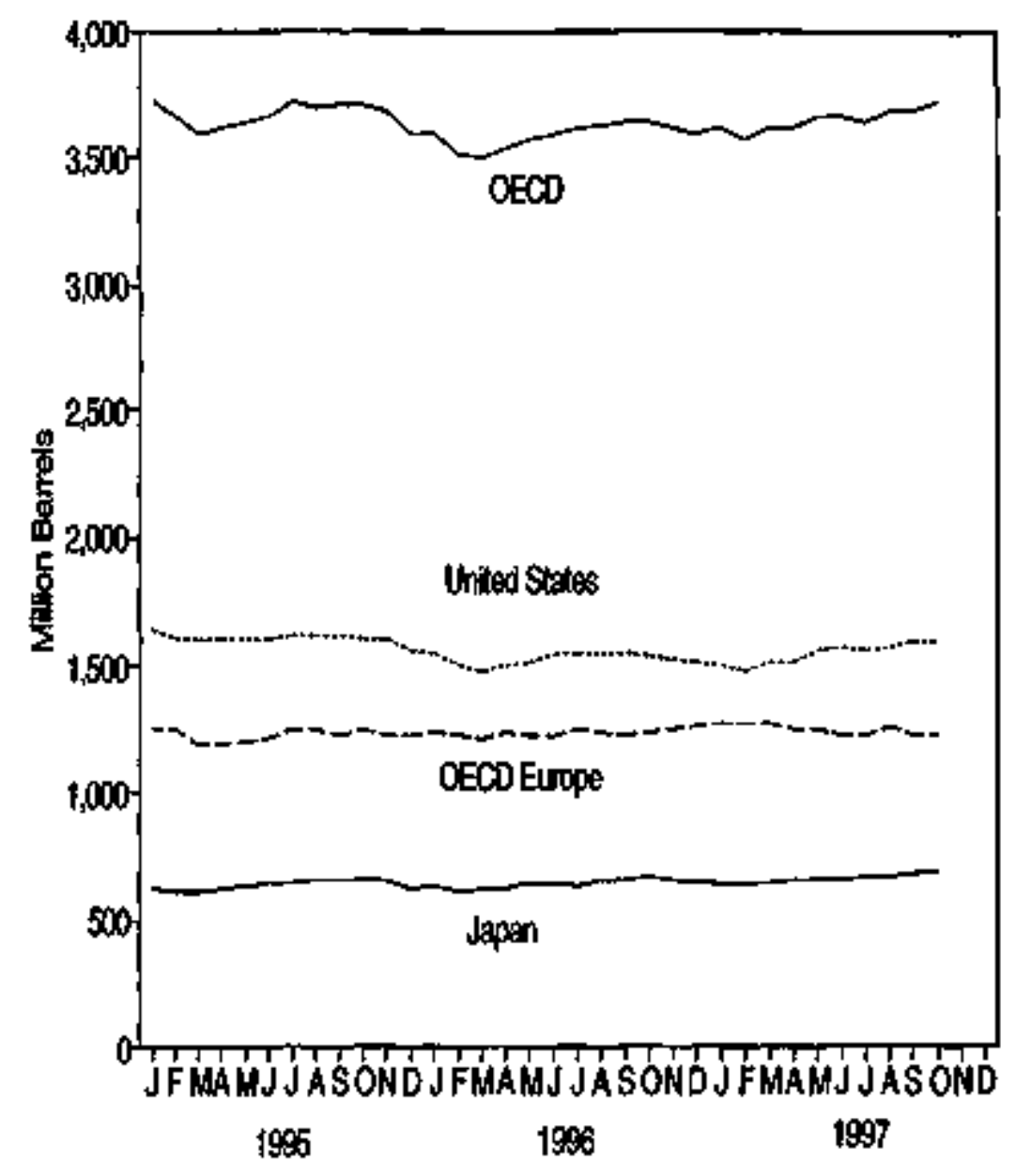

Source: Table 1.5 


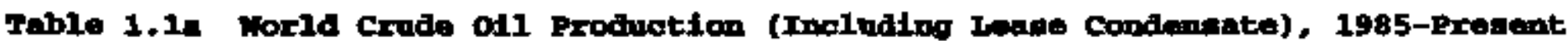
(Fhoplend Barcoll pwr Dar)

\begin{tabular}{|c|c|c|c|c|c|c|c|c|c|c|c|c|c|}
\hline & & Mlgorta & $\begin{array}{l}\text { Thap- } \\
\text { netis }\end{array}$ & Iran & Iritag & tromeite & Libye & Nigoria & attar & $\begin{array}{l}\text { Anudi } \\
\text { arebiast }\end{array}$ & $\begin{array}{l}\text { Dolted } \\
\text { Nrib } \\
\text { mitretes }\end{array}$ & $\begin{array}{l}\text { Veno: } \\
\text { zueis }\end{array}$ & $\begin{array}{l}\text { Toten } \\
\text { otris }\end{array}$ \\
\hline $\begin{array}{l}1985 \\
1996 \\
1987 \\
1988 \\
1989 \\
1990 \\
1991 \\
1992 \\
1993 \\
1994 \\
1995\end{array}$ & 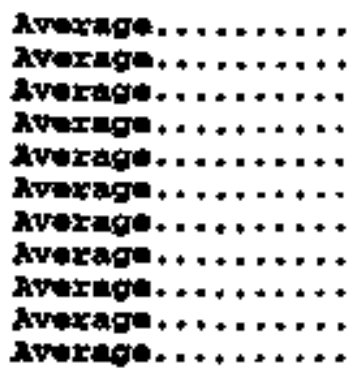 & $\begin{array}{l}1,097 \\
1,045 \\
1,040 \\
1,040 \\
1,095 \\
1,175 \\
1,230 \\
1,214 \\
1,162 \\
1,180 \\
1,202\end{array}$ & $\begin{array}{l}1,325 \\
1,390 \\
1,343 \\
1,342 \\
1,409 \\
1,462 \\
1,592 \\
1,504 \\
1,511 \\
1,510 \\
1,503\end{array}$ & $\begin{array}{l}2,250 \\
2,035 \\
2,290 \\
2,240 \\
2,810 \\
3,080 \\
3,317 \\
3,439 \\
3,540 \\
3,618 \\
3,643\end{array}$ & $\begin{array}{r}1,433 \\
1,690 \\
2,079 \\
2,685 \\
2,897 \\
2,040 \\
305 \\
425 \\
512 \\
553 \\
560\end{array}$ & $\begin{array}{l}1,023 \\
1,419 \\
1,585 \\
1,492 \\
1,783 \\
1,175 \\
190 \\
1,050 \\
1,852 \\
2,025 \\
2,057\end{array}$ & $\begin{array}{l}1,059 \\
1,034 \\
972 \\
1,175 \\
1,150 \\
1,375 \\
1,483 \\
1,433 \\
1,361 \\
1,379 \\
1,390\end{array}$ & $\begin{array}{l}1,495 \\
1,467 \\
1,341 \\
1,450 \\
1,716 \\
1,010 \\
1,992 \\
1,943 \\
1,960 \\
1,931 \\
1,993\end{array}$ & $\begin{array}{l}301 \\
309 \\
293 \\
346 \\
380 \\
406 \\
395 \\
433 \\
413 \\
415 \\
483\end{array}$ & $\begin{array}{l}3,388 \\
4,870 \\
4,265 \\
5,016 \\
5,064 \\
6,410 \\
0,115 \\
8,332 \\
8,198 \\
8,120 \\
8,231\end{array}$ & $\begin{array}{l}1,193 \\
1,334 \\
2,511 \\
1,565 \\
1,960 \\
2,117 \\
2,386 \\
2,266 \\
2,159 \\
2,193 \\
2,279\end{array}$ & $\begin{array}{l}1,677 \\
1,787 \\
1,752 \\
1,903 \\
1,907 \\
2,137 \\
2,375 \\
2,371 \\
2,050 \\
2,588 \\
2,750\end{array}$ & $\begin{array}{l}16,181 \\
18,275 \\
18,517 \\
20,324 \\
22,071 \\
23,195 \\
23,275 \\
24,398 \\
25,119 \\
25,510 \\
26,092\end{array}$ \\
\hline 1996 & 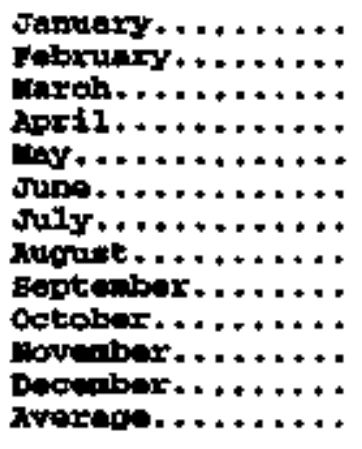 & $\begin{array}{l}1,220 \\
1,220 \\
1,210 \\
1,230 \\
1,245 \\
1,250 \\
1,250 \\
1,250 \\
1,250 \\
1,260 \\
1,260 \\
1,260 \\
1,242\end{array}$ & $\begin{array}{l}1,540 \\
1,540 \\
1,540 \\
1,530 \\
1,530 \\
1,550 \\
1,520 \\
1,540 \\
1,560 \\
1,590 \\
1,570 \\
1,570 \\
1,547\end{array}$ & $\begin{array}{l}3,735 \\
3,685 \\
3,715 \\
3,685 \\
3,635 \\
3,685 \\
3,685 \\
3,715 \\
3,735 \\
3,635 \\
3,685 \\
3,695 \\
3,686\end{array}$ & $\begin{array}{l}555 \\
555 \\
555 \\
555 \\
555 \\
555 \\
555 \\
555 \\
555 \\
555 \\
555 \\
995 \\
584\end{array}$ & $\begin{array}{l}2,038 \\
2,057 \\
2,057 \\
2,067 \\
2,055 \\
2,065 \\
2,065 \\
2,000 \\
2,070 \\
2,075 \\
2,075 \\
2,077 \\
2,062\end{array}$ & $\begin{array}{l}1,400 \\
1,400 \\
1,400 \\
1,400 \\
1,400 \\
1,400 \\
1,400 \\
1,400 \\
1,400 \\
1,400 \\
1,400 \\
1,410 \\
1,401\end{array}$ & $\begin{array}{l}2,160 \\
2,180 \\
2,190 \\
2,160 \\
2,200 \\
2,200 \\
2,170 \\
2,190 \\
2,150 \\
2,220 \\
2,220 \\
2,225 \\
2,180\end{array}$ & $\begin{array}{l}500 \\
500 \\
500 \\
505 \\
505 \\
505 \\
505 \\
505 \\
525 \\
525 \\
505 \\
545 \\
510\end{array}$ & $\begin{array}{l}8,118 \\
6,248 \\
8,248 \\
8,088 \\
0,135 \\
8,195 \\
8,295 \\
8,220 \\
8,200 \\
8,255 \\
8,255 \\
8,358 \\
0,218\end{array}$ & $\begin{array}{l}2,290 \\
2,265 \\
2,285 \\
2,250 \\
2,275 \\
2,270 \\
2,260 \\
2,260 \\
2,310 \\
2,310 \\
2,250 \\
2,305 \\
2,278\end{array}$ & $\begin{array}{l}2,940 \\
2,940 \\
2,990 \\
2,990 \\
2,990 \\
2,990 \\
3,040 \\
3,090 \\
3,090 \\
3,140 \\
3,190 \\
3,240 \\
3,053\end{array}$ & $\begin{array}{l}26,495 \\
26,590 \\
26,690 \\
26,460 \\
26,525 \\
26,665 \\
26,745 \\
26,765 \\
26,845 \\
26,945 \\
26,965 \\
27,520 \\
26,769\end{array}$ \\
\hline 1997 & 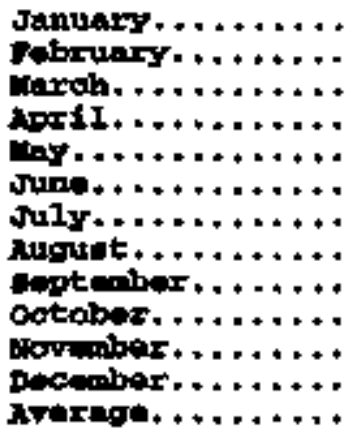 & $\begin{array}{l}1,260 \\
1,270 \\
1,280 \\
1,280 \\
1,280 \\
1,260 \\
1,280 \\
1,280 \\
1,280 \\
1,280 \\
1,280 \\
1,290 \\
1,277\end{array}$ & $\begin{array}{l}1,570 \\
1,590 \\
1,600 \\
1,560 \\
1,590 \\
1,530 \\
1,590 \\
1,530 \\
1,490 \\
1,490 \\
1,540 \\
1,540 \\
1,546\end{array}$ & $\begin{array}{l}3,685 \\
3,685 \\
3,685 \\
3,685 \\
3,635 \\
3,735 \\
3,685 \\
3,685 \\
3,485 \\
3,635 \\
3,685 \\
3,685 \\
3,664\end{array}$ & $\begin{array}{r}1,085 \\
1,125 \\
1,175 \\
1,275 \\
1,325 \\
605 \\
605 \\
1,515 \\
1,735 \\
1,625 \\
1,390 \\
1,181\end{array}$ & $\begin{array}{l}2,085 \\
2,077 \\
2,105 \\
2,107 \\
2,027 \\
2,050 \\
2,070 \\
2,070 \\
2,075 \\
2,075 \\
2,075 \\
2,175 \\
2,083\end{array}$ & $\begin{array}{l}1,430 \\
1,490 \\
1,440 \\
1,450 \\
1,450 \\
1,450 \\
1,450 \\
1,450 \\
1,450 \\
1,450 \\
1,450 \\
1,450 \\
1,446\end{array}$ & $\begin{array}{r}12,280 \\
2,310 \\
2,240 \\
2,310 \\
2,270 \\
2,340 \\
2,330 \\
2,350 \\
2,300 \\
2,400 \\
2,360 \\
2,320 \\
2,317\end{array}$ & $\begin{array}{l}585 \\
585 \\
585 \\
585 \\
605 \\
690 \\
685 \\
685 \\
685 \\
685 \\
705 \\
705 \\
649\end{array}$ & $\begin{array}{l}8,265 \\
8,408 \\
8,513 \\
8,568 \\
8,548 \\
8,540 \\
8,560 \\
8,660 \\
0,665 \\
8,665 \\
6,615 \\
8,725 \\
8,562\end{array}$ & $\begin{array}{l}2,300 \\
2,330 \\
2,360 \\
2,360 \\
2,310 \\
2,325 \\
2,325 \\
2,325 \\
2,325 \\
2,325 \\
2,305 \\
2,310 \\
2,316\end{array}$ & $\begin{array}{l}3,190 \\
3,190 \\
3,200 \\
3,220 \\
3,240 \\
3,250 \\
3,270 \\
3,390 \\
3,430 \\
3,430 \\
3,460 \\
3,490 \\
3,315\end{array}$ & $\begin{array}{r}\mathrm{R27}, 735 \\
28,000 \\
28,195 \\
28,100 \\
28,170 \\
\mathrm{R27}, 795 \\
\mathrm{R27}, 790 \\
28,940 \\
28,920 \\
29,060 \\
28,865 \\
28,971 \\
28,361\end{array}$ \\
\hline
\end{tabular}

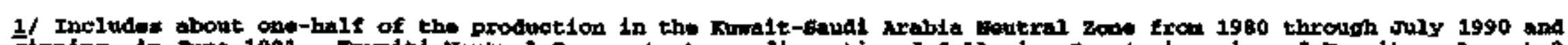

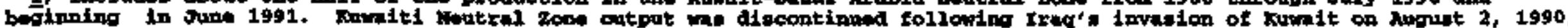

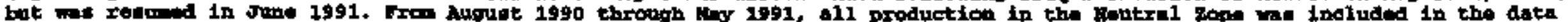

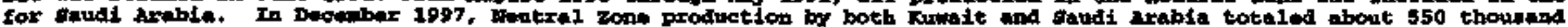
britela por day.

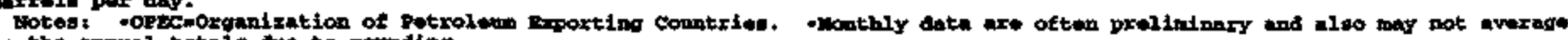
to the ennual totalis dite to founding.

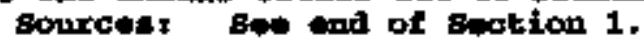


Table 1. Ib Worla Cruap oil production (Including Inape Conaminte), 1985-Pxesent (Thoonand Binnele par Dat)

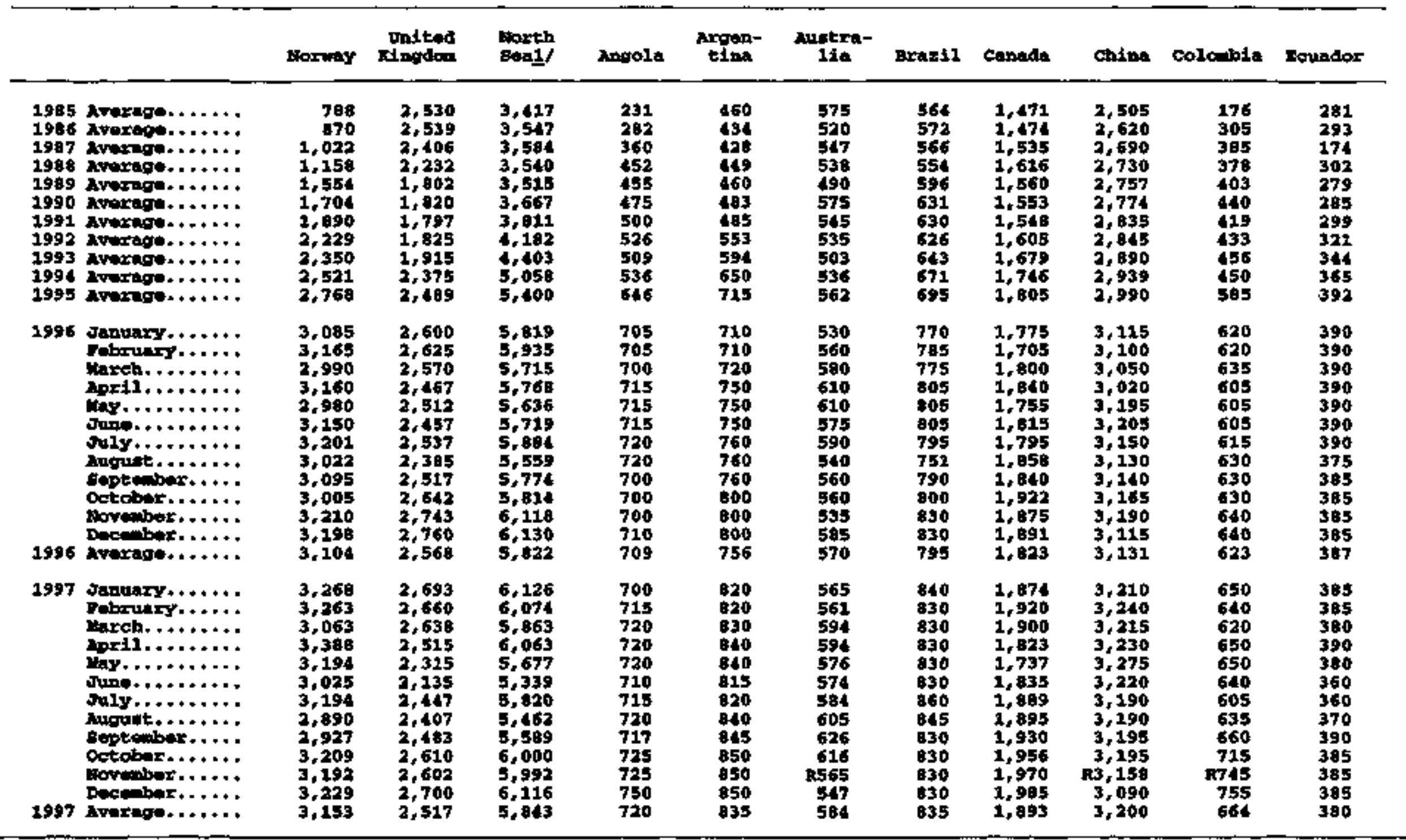

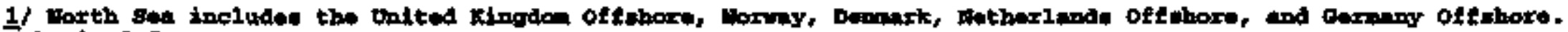

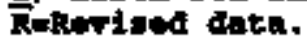

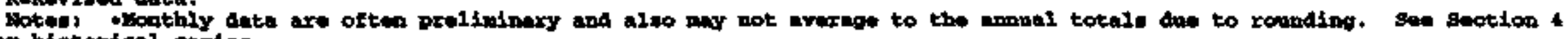
for hivtorienl wien.

sources: tos end of enction 1. 


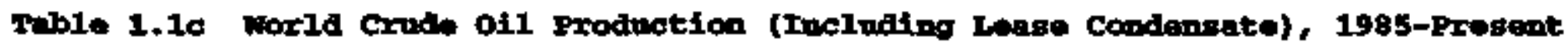
(Whourand Barrwil par Day)

\begin{tabular}{|c|c|c|c|c|c|c|c|c|c|c|c|c|c|}
\hline & & Espt & Gabon & Indite & Holayela & Nenciloo & Onas & Apas 1 a & $\begin{array}{l}\text { For.ar } \\
\text { v.8.8.R. }\end{array}$ & Byxt & $\begin{array}{l}\text { Onited } \\
\text { statates }\end{array}$ & othor 1 & / Wor14 \\
\hline $\begin{array}{l}1985 \\
1986 \\
1987 \\
1988 \\
1989 \\
1990 \\
1991 \\
1992 \\
1993 \\
1994 \\
1995\end{array}$ & 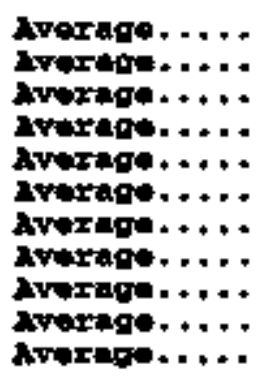 & $\begin{array}{l}867 \\
813 \\
896 \\
948 \\
965 \\
973 \\
974 \\
981 \\
890 \\
896 \\
920\end{array}$ & $\begin{array}{l}172 \\
166 \\
155 \\
159 \\
209 \\
270 \\
294 \\
298 \\
313 \\
329 \\
345\end{array}$ & $\begin{array}{l}520 \\
630 \\
609 \\
635 \\
700 \\
680 \\
615 \\
581 \\
534 \\
590 \\
703\end{array}$ & $\begin{array}{l}440 \\
504 \\
497 \\
540 \\
585 \\
619 \\
646 \\
653 \\
640 \\
645 \\
692\end{array}$ & $\begin{array}{l}2,745 \\
2,495 \\
2,548 \\
2,512 \\
2,520 \\
2,553 \\
2,690 \\
2,669 \\
2,673 \\
2,685 \\
2,618\end{array}$ & $\begin{array}{l}498 \\
560 \\
582 \\
617 \\
641 \\
685 \\
700 \\
740 \\
776 \\
810 \\
851\end{array}$ & $\begin{array}{r}=- \\
=- \\
=- \\
=- \\
=- \\
7,632 \\
6,730 \\
6,135 \\
5,995\end{array}$ & $\begin{array}{r}11,585 \\
11,895 \\
12,050 \\
12,053 \\
11,715 \\
10,975 \\
9,992 \\
=- \\
=- \\
=-\end{array}$ & $\begin{array}{l}178 \\
194 \\
290 \\
265 \\
340 \\
388 \\
492 \\
481 \\
554 \\
560 \\
610\end{array}$ & $\begin{array}{l}0,971 \\
9,680 \\
8,349 \\
0,140 \\
7,613 \\
7,355 \\
7,117 \\
7,171 \\
6,847 \\
6,662 \\
6,560\end{array}$ & $\begin{array}{l}2,124 \\
3,166 \\
3,120 \\
2,235 \\
2,249 \\
2,253 \\
2,274 \\
3,233 \\
3,290 \\
3,393 \\
3,423\end{array}$ & $\begin{array}{l}53,962 \\
54,227 \\
56,666 \\
59,737 \\
59,063 \\
50,566 \\
60,207 \\
60,216 \\
60,247 \\
61,003 \\
62,446\end{array}$ \\
\hline 2996 & 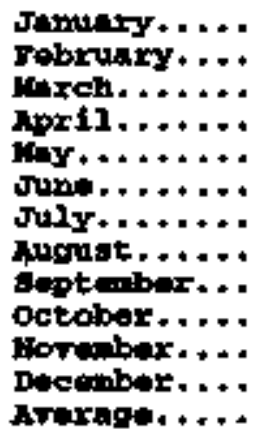 & $\begin{array}{l}920 \\
920 \\
920 \\
920 \\
920 \\
920 \\
920 \\
920 \\
920 \\
920 \\
930 \\
930 \\
922\end{array}$ & $\begin{array}{l}360 \\
360 \\
370 \\
370 \\
370 \\
370 \\
370 \\
370 \\
370 \\
370 \\
370 \\
370 \\
368\end{array}$ & $\begin{array}{l}675 \\
700 \\
665 \\
685 \\
660 \\
650 \\
625 \\
625 \\
625 \\
625 \\
630 \\
650 \\
651\end{array}$ & $\begin{array}{l}690 \\
680 \\
680 \\
700 \\
700 \\
700 \\
700 \\
700 \\
700 \\
700 \\
700 \\
700 \\
695\end{array}$ & $\begin{array}{l}2,795 \\
2,800 \\
2,870 \\
2,860 \\
2,875 \\
2,880 \\
2,870 \\
2,830 \\
2,860 \\
2,860 \\
2,860 \\
2,900 \\
2,055\end{array}$ & $\begin{array}{l}865 \\
865 \\
870 \\
880 \\
880 \\
880 \\
985 \\
885 \\
990 \\
900 \\
900 \\
900 \\
883\end{array}$ & $\begin{array}{l}5,763 \\
5,667 \\
5,755 \\
5,763 \\
5,789 \\
5,763 \\
5,737 \\
5,780 \\
5,750 \\
5,737 \\
5,632 \\
5,755 \\
5,774\end{array}$ & $\begin{array}{l}=- \\
=- \\
= \\
=- \\
=- \\
= \\
=- \\
=- \\
= \\
=\end{array}$ & $\begin{array}{l}605 \\
605 \\
605 \\
600 \\
600 \\
600 \\
600 \\
600 \\
600 \\
600 \\
610 \\
620 \\
604\end{array}$ & $\begin{array}{l}6,495 \\
6,577 \\
6,571 \\
6,414 \\
6,394 \\
6,458 \\
6,338 \\
6,360 \\
6,492 \\
6,481 \\
6,476 \\
6,506 \\
6,465\end{array}$ & $\begin{array}{l}3,421 \\
3,447 \\
3,419 \\
3,455 \\
3,448 \\
3,452 \\
3,553 \\
3,559 \\
3,564 \\
3,652 \\
3,656 \\
3,651 \\
3,520\end{array}$ & $\begin{array}{l}63,375 \\
63,776 \\
63,625 \\
63,400 \\
63,478 \\
63,805 \\
63,896 \\
63,566 \\
64,023 \\
64,379 \\
64,937 \\
65,416 \\
63,972\end{array}$ \\
\hline 1997 & 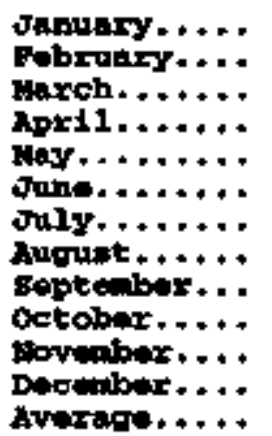 & $\begin{array}{l}685 \\
685 \\
890 \\
890 \\
880 \\
970 \\
980 \\
970 \\
660 \\
660 \\
660 \\
660 \\
974\end{array}$ & $\begin{array}{l}370 \\
370 \\
370 \\
370 \\
370 \\
370 \\
370 \\
370 \\
360 \\
370 \\
370 \\
370 \\
369\end{array}$ & $\begin{array}{r}650 \\
650 \\
660 \\
685 \\
685 \\
685 \\
685 \\
685 \\
675 \\
2678 \\
1681 \\
681 \\
675\end{array}$ & $\begin{array}{l}730 \\
730 \\
730 \\
750 \\
750 \\
740 \\
740 \\
740 \\
760 \\
760 \\
760 \\
760 \\
746\end{array}$ & $\begin{array}{l}2,940 \\
2,970 \\
2,970 \\
2,945 \\
2,990 \\
3,005 \\
3,035 \\
3,090 \\
3,105 \\
3,087 \\
3,085 \\
3,090 \\
3,026\end{array}$ & $\begin{array}{l}890 \\
900 \\
900 \\
900 \\
890 \\
900 \\
890 \\
870 \\
670 \\
890 \\
890 \\
890 \\
\text { 895 }\end{array}$ & $\begin{array}{l}5,789 \\
5,729 \\
5,772 \\
5,993 \\
5,902 \\
5,902 \\
5,923 \\
5,945 \\
5,958 \\
5,954 \\
5,945 \\
5,893 \\
5,884\end{array}$ & $\begin{array}{l}= \\
= \\
= \\
= \\
= \\
= \\
= \\
= \\
= \\
= \\
=\end{array}$ & $\begin{array}{l}\mathbf{5 6 5} \\
\mathbf{5 6 5} \\
\mathbf{5 6 5} \\
\mathbf{5 6 0} \\
\mathbf{5 6 0} \\
\mathbf{5 6 0} \\
\mathbf{5 6 0} \\
\mathbf{5 6 0} \\
\mathbf{5 6 0} \\
\mathbf{5 6 0} \\
\mathbf{5 6 0} \\
\mathbf{5 6 0} \\
\mathbf{5 6 1}\end{array}$ & 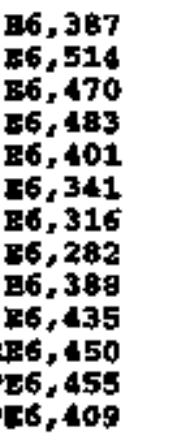 & $\begin{array}{r}3,706 \quad \\
3,685 \\
3,725 \\
3,747 \\
3,711 \\
3,716 \mathrm{R} \\
3,670 \\
3,663 \\
13,664 \mathrm{~B} \\
\mathrm{13}, 678 \mathrm{R} \\
33,694 \mathrm{R} \\
3,665 \\
3,694\end{array}$ & $\begin{array}{r}\mathrm{H} 65,652 \\
66,031 \\
66,027 \\
66,603 \\
65,825 \\
\mathrm{RE5}, 018 \\
\mathrm{R} 65,473 \\
66,402 \\
\mathrm{R} 66,723 \\
\mathrm{R} 67,413 \\
\mathrm{RE7}, 182 \\
66,916 \\
66,264\end{array}$ \\
\hline
\end{tabular}

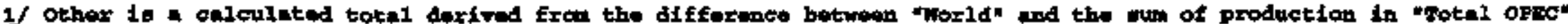

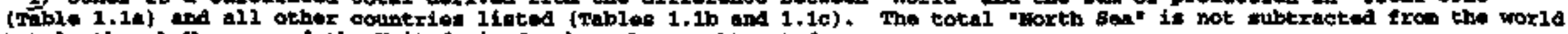
total, though Horway and the United Kingda. have boen ruberacted.

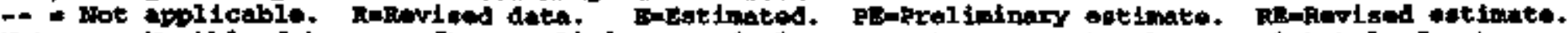

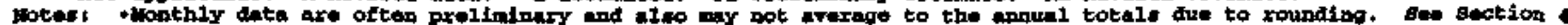
for historidel rerita.

pourate: Bes ond of suction 1. 


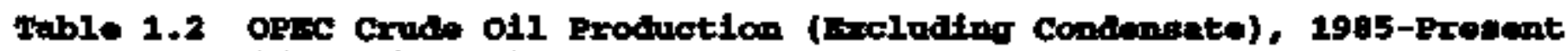

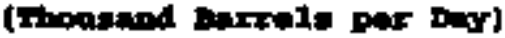

\begin{tabular}{|c|c|c|c|c|c|c|c|c|c|c|c|c|c|}
\hline & & Mlverde & $\begin{array}{l}\text { Indo- } \\
\text { notis }\end{array}$ & $\operatorname{IxnR}$ & Irng & Kunnitelf & bibre & MAgerde & ortex & $\begin{array}{l}\text { Eaudl } \\
\text { xrabtal }\end{array}$ & $\begin{array}{l}\text { Onteed } \\
\text { Axeb } \\
\text { ronfrater }\end{array}$ & $\begin{array}{l}\text { Votan- } \\
\text { zuilia }\end{array}$ & $\begin{array}{l}\text { Totel } \\
\text { Opix }\end{array}$ \\
\hline $\begin{array}{l}1965 \\
1906 \\
1997 \\
1980 \\
1999 \\
1990 \\
1991 \\
1992 \\
1993 \\
1994 \\
1995\end{array}$ & 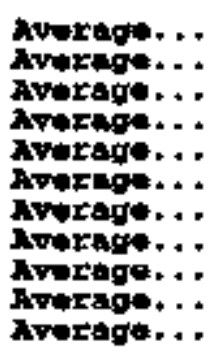 & $\begin{array}{l}702 \\
600 \\
648 \\
640 \\
690 \\
765 \\
600 \\
772 \\
767 \\
750 \\
767\end{array}$ & $\begin{array}{l}1,181 \\
1,257 \\
1,189 \\
1,177 \\
1,231 \\
1,381 \\
1,410 \\
1,326 \\
1,327 \\
1,334 \\
1,343\end{array}$ & $\begin{array}{l}2,250 \\
2,035 \\
2,298 \\
2,240 \\
2,610 \\
3,098 \\
3,312 \\
3,429 \\
3,540 \\
3,600 \\
3,600\end{array}$ & $\begin{array}{r}1,433 \\
1,690 \\
2,079 \\
2,685 \\
2,897 \\
2,040 \\
305 \\
425 \\
512 \\
548 \\
550\end{array}$ & $\begin{array}{l}1,023 \\
1,419 \\
1,585 \\
1,492 \\
1,783 \\
1,175 \\
1,058 \\
1,058 \\
2,034 \\
2,057\end{array}$ & $\begin{array}{r}1,059 \\
1,034 \\
1,972 \\
1,175 \\
1,150 \\
1,375 \\
1,483 \\
1,493 \\
1,361 \\
1,378 \\
1,390\end{array}$ & $\begin{array}{l}1,475 \\
1,447 \\
1,291 \\
1,400 \\
1,666 \\
1,758 \\
1,832 \\
1,030 \\
1,835 \\
1,087 \\
1,876\end{array}$ & $\begin{array}{l}301 \\
306 \\
293 \\
346 \\
380 \\
406 \\
395 \\
423 \\
413 \\
409 \\
449\end{array}$ & $\begin{array}{r}3,380 \\
4,870 \\
4,265 \\
5,096 \\
5,064 \\
6,410 \\
0,115 \\
8,337 \\
9,198 \\
8,147 \\
0,231\end{array}$ & $\begin{array}{l}1,133 \\
1,278 \\
1,441 \\
1,465 \\
1,783 \\
2,066 \\
2,326 \\
2,206 \\
2,109 \\
2,183 \\
2,197\end{array}$ & $\begin{array}{l}1,558 \\
1,645 \\
1,587 \\
1,715 \\
1,747 \\
2,097 \\
2,330 \\
2,334 \\
3,110 \\
2,562 \\
3,710\end{array}$ & $\begin{array}{l}15,503 \\
17,575 \\
17,640 \\
19,423 \\
21,201 \\
32,458 \\
22,506 \\
23,558 \\
34,304 \\
24,512 \\
25,179\end{array}$ \\
\hline 1996 & 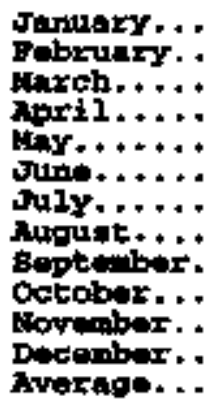 & $\begin{array}{l}790 \\
790 \\
780 \\
810 \\
815 \\
820 \\
820 \\
820 \\
820 \\
830 \\
830 \\
830 \\
812\end{array}$ & $\begin{array}{l}1,360 \\
1,360 \\
1,360 \\
1,350 \\
1,350 \\
1,370 \\
1,340 \\
1,360 \\
1,380 \\
1,300 \\
1,390 \\
1,390 \\
1,367\end{array}$ & $\begin{array}{l}3,700 \\
3,650 \\
3,680 \\
3,650 \\
3,600 \\
3,650 \\
3,650 \\
3,680 \\
3,700 \\
3,600 \\
3,650 \\
3,600 \\
3,651\end{array}$ & $\begin{array}{l}550 \\
550 \\
550 \\
550 \\
550 \\
550 \\
550 \\
550 \\
550 \\
550 \\
550 \\
890 \\
579\end{array}$ & $\begin{array}{l}2,038 \\
2,057 \\
2,057 \\
2,067 \\
2,055 \\
2,065 \\
2,065 \\
2,010 \\
2,070 \\
2,075 \\
2,075 \\
2,077 \\
2,062\end{array}$ & $\begin{array}{l}1,400 \\
1,400 \\
1,400 \\
1,400 \\
1,400 \\
2,400 \\
1,400 \\
1,400 \\
1,400 \\
1,400 \\
1,400 \\
1,410 \\
1,401\end{array}$ & $\begin{array}{l}2,010 \\
2,030 \\
2,040 \\
2,010 \\
2,050 \\
2,050 \\
2,020 \\
3,040 \\
2,000 \\
2,114 \\
2,120 \\
2,125 \\
2,051\end{array}$ & $\begin{array}{l}465 \\
465 \\
465 \\
470 \\
470 \\
470 \\
470 \\
470 \\
490 \\
490 \\
470 \\
540 \\
475\end{array}$ & $\begin{array}{l}0,218 \\
8,248 \\
0,288 \\
6,018 \\
6,135 \\
8,195 \\
6,295 \\
6,200 \\
6,200 \\
6,255 \\
9,255 \\
0,358 \\
0,218\end{array}$ & $\begin{array}{l}2,210 \\
2,195 \\
2,205 \\
2,170 \\
2,195 \\
2,190 \\
2,180 \\
2,100 \\
2,230 \\
2,230 \\
2,170 \\
2,225 \\
2,198\end{array}$ & $\begin{array}{l}2,900 \\
2,9700 \\
2,950 \\
2,950 \\
2,950 \\
2,950 \\
3,000 \\
3,050 \\
3,050 \\
3,200 \\
3,150 \\
3,200 \\
3,013\end{array}$ & $\begin{array}{l}25,540 \\
25,635 \\
25,735 \\
25,505 \\
25,570 \\
25,710 \\
25,790 \\
25,920 \\
25,090 \\
26,040 \\
26,060 \\
26,625 \\
25,026\end{array}$ \\
\hline 1997 & 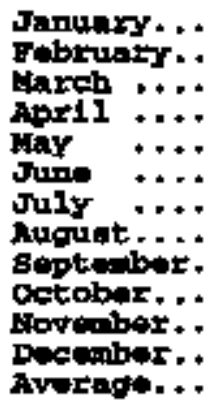 & $\begin{array}{l}830 \\
940 \\
850 \\
850 \\
850 \\
830 \\
850 \\
850 \\
850 \\
850 \\
850 \\
860 \\
847\end{array}$ & $\begin{array}{l}1,390 \\
1,410 \\
1,420 \\
1,380 \\
1,400 \\
1,354 \\
1,350 \\
1,354 \\
1,310 \\
1,310 \\
1,360 \\
1,360 \\
1,366\end{array}$ & $\begin{array}{l}3,650 \\
3,650 \\
3,650 \\
3,650 \\
3,600 \\
3,700 \\
3,650 \\
3,650 \\
3,650 \\
3,600 \\
3,650 \\
3,650 \\
3,629\end{array}$ & $\begin{array}{r}1,080 \\
1,2120 \\
1,170 \\
1,270 \\
1,320 \\
600 \\
600 \\
1,510 \\
1,730 \\
1,620 \\
1,385 \\
1,776\end{array}$ & $\begin{array}{l}2,085 \\
2,077 \\
2,105 \\
2,107 \\
2,027 \\
2,050 \\
2,070 \\
2,070 \\
2,075 \\
2,075 \\
2,075 \\
2,175 \\
2,083\end{array}$ & $\begin{array}{l}1,430 \\
2,430 \\
1,440 \\
1,450 \\
1,450 \\
1,450 \\
1,450 \\
1,450 \\
1,450 \\
1,450 \\
1,450 \\
1,450 \\
1,446\end{array}$ & $\begin{array}{r}\mathrm{R2}, 180 \\
2,210 \\
2,140 \\
2,210 \\
2,170 \\
\mathrm{R}, 200 \\
1,230 \\
2,250 \\
2,240 \\
2,300 \\
2,260 \\
2,220 \\
2,217\end{array}$ & $\begin{array}{l}550 \\
550 \\
550 \\
550 \\
570 \\
655 \\
650 \\
650 \\
650 \\
650 \\
670 \\
670 \\
614\end{array}$ & $\begin{array}{l}9,265 \\
9,408 \\
8,515 \\
9,568 \\
8,518 \\
8,540 \\
9,560 \\
9,660 \\
9,665 \\
9,665 \\
9,615 \\
8,725 \\
9,562\end{array}$ & $\begin{array}{l}2,220 \\
2,250 \\
2,280 \\
2,280 \\
2,230 \\
2,245 \\
2,245 \\
2,245 \\
2,245 \\
2,245 \\
2,225 \\
2,230 \\
2,236\end{array}$ & $\begin{array}{r}3,150 \\
3,150 \\
3,160 \\
3,180 \\
3.200 \\
3,220 \\
3,230 \\
3,350 \\
3,390 \\
3,390 \\
3,420 \\
3.450 \\
3,375\end{array}$ & $\begin{array}{r}\mathrm{R} 26,830 \\
27,095 \\
27,280 \\
27,495 \\
27,265 \\
\mathrm{R26}, 880 \\
\mathrm{R26}, 885 \\
28,035 \\
28,015 \\
28,155 \\
27,960 \\
27,566 \\
27,457\end{array}$ \\
\hline
\end{tabular}

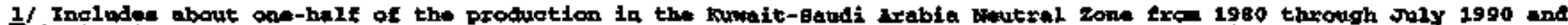

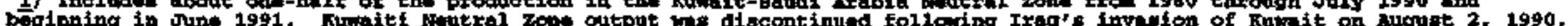

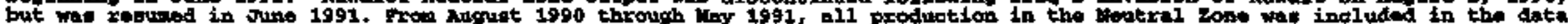

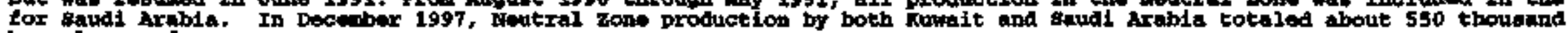
barxelis per day.

Rakryl ted Date.

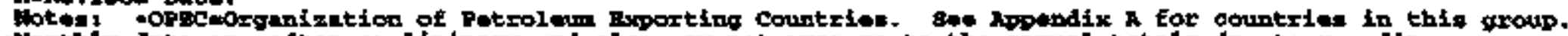

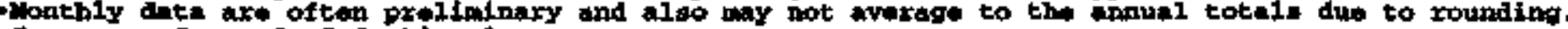

Dourdest Bene of of anction 1 
Table 1.3 World fatural Gas plent Liquids production, 1985-Pxement (Thousend Eaxrele par Doy)

\begin{tabular}{|c|c|c|c|c|c|c|c|c|c|c|c|c|}
\hline & & Algerta & Canata & bedion & $\begin{array}{l}\text { gaudi } \\
\text { srubin }\end{array}$ & numpis & Pownty & $\begin{array}{l}\text { Ontted } \\
\text { Stated }\end{array}$ & Ferotur & onries/ & ortect & Wor14 \\
\hline $\begin{array}{l}1965 \\
1906 \\
1987 \\
1989 \\
1989 \\
1990 \\
1991 \\
1992 \\
1993 \\
1994 \\
1995\end{array}$ & 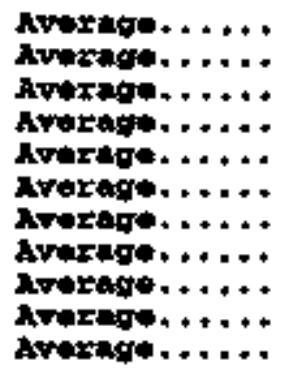 & $\begin{array}{l}120 \\
120 \\
140 \\
120 \\
130 \\
130 \\
140 \\
140 \\
145 \\
140 \\
145\end{array}$ & $\begin{array}{l}337 \\
328 \\
367 \\
981 \\
410 \\
426 \\
431 \\
460 \\
506 \\
529 \\
581\end{array}$ & $\begin{array}{l}271 \\
352 \\
338 \\
370 \\
384 \\
428 \\
457 \\
454 \\
459 \\
461 \\
447\end{array}$ & $\begin{array}{l}375 \\
385 \\
418 \\
499 \\
503 \\
620 \\
680 \\
713 \\
704 \\
698 \\
701\end{array}$ & $\begin{array}{l}=- \\
=- \\
=- \\
=- \\
=- \\
230 \\
2 \geq 0 \\
200 \\
100\end{array}$ & $\begin{array}{l}350 \\
440 \\
430 \\
450 \\
425 \\
425 \\
420 \\
=0 \\
=- \\
=-\end{array}$ & $\begin{array}{l}1,609 \\
1,551 \\
1,595 \\
1,625 \\
1,546 \\
1,559 \\
1,659 \\
1,697 \\
1,736 \\
1,727 \\
1,762\end{array}$ & $\begin{array}{r}645 \\
700 \\
721 \\
800 \\
051 \\
930 \\
931 \\
1,003 \\
1,040 \\
1,071 \\
1,106\end{array}$ & $\begin{array}{r}605 \\
860 \\
9040 \\
979 \\
1,041 \\
1,107 \\
1,119 \\
1,185 \\
1,230 \\
1,267 \\
1,301\end{array}$ & $\begin{array}{r}893 \\
969 \\
1,006 \\
1,077 \\
1,188 \\
1,281 \\
1,299 \\
1,364 \\
1,435 \\
1,465 \\
1,506\end{array}$ & $\begin{array}{r}3,938 \\
4,150 \\
4,279 \\
4,481 \\
4,502 \\
4,632 \\
4,827 \\
4,973 \\
3,169 \\
5,282 \\
5,474\end{array}$ \\
\hline 1996 & 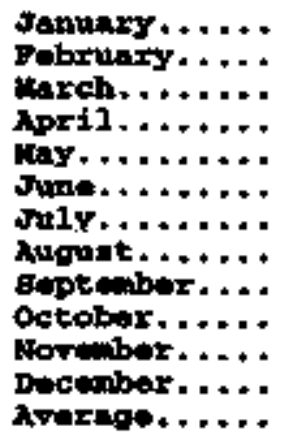 & $\begin{array}{l}145 \\
145 \\
145 \\
145 \\
145 \\
145 \\
145 \\
145 \\
145 \\
145 \\
145 \\
145 \\
145\end{array}$ & $\begin{array}{l}585 \\
545 \\
615 \\
615 \\
565 \\
560 \\
610 \\
619 \\
595 \\
620 \\
615 \\
640 \\
599\end{array}$ & $\begin{array}{l}470 \\
475 \\
475 \\
475 \\
475 \\
485 \\
440 \\
335 \\
340 \\
370 \\
370 \\
370 \\
473\end{array}$ & $\begin{array}{l}690 \\
700 \\
700 \\
690 \\
690 \\
695 \\
705 \\
700 \\
695 \\
695 \\
695 \\
705 \\
697\end{array}$ & $\begin{array}{l}248 \\
248 \\
248 \\
248 \\
248 \\
248 \\
248 \\
248 \\
248 \\
248 \\
248 \\
248 \\
240\end{array}$ & $\begin{array}{l}=- \\
=- \\
=- \\
=- \\
=- \\
=- \\
=- \\
=- \\
=- \\
=\end{array}$ & $\begin{array}{l}1,716 \\
1,680 \\
1,814 \\
1,845 \\
1,806 \\
1,033 \\
1,829 \\
1,850 \\
1,872 \\
1,912 \\
1,915 \\
1,876 \\
1,830\end{array}$ & $\begin{array}{l}1,075 \\
1,085 \\
1,085 \\
1,075 \\
1,075 \\
1,000 \\
1,090 \\
1,085 \\
1,080 \\
1,080 \\
1,080 \\
1,090 \\
1,082\end{array}$ & $\begin{array}{l}1,267 \\
1,277 \\
1,277 \\
1,267 \\
1,267 \\
1,272 \\
1,282 \\
1,277 \\
1,272 \\
1,272 \\
1,272 \\
1,282 \\
1,274\end{array}$ & $\begin{array}{l}1,445 \\
1,455 \\
1,455 \\
1,445 \\
1,445 \\
1,450 \\
1,450 \\
1,455 \\
1,450 \\
1,450 \\
1,450 \\
1,460 \\
1,452\end{array}$ & $\begin{array}{l}5,530 \\
5,483 \\
5,679 \\
5,687 \\
5,609 \\
5,625 \\
5,649 \\
5,549 \\
5,564 \\
5,720 \\
5,652 \\
5,709 \\
5,625\end{array}$ \\
\hline 1987 & 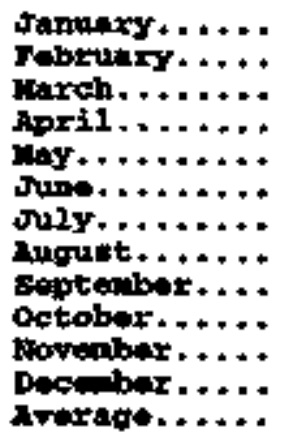 & $\begin{array}{l}145 \\
145 \\
145 \\
145 \\
145 \\
145 \\
145 \\
145 \\
145 \\
145 \\
145 \\
145 \\
145\end{array}$ & $\begin{array}{l}660 \\
645 \\
652 \\
610 \\
597 \\
595 \\
598 \\
625 \\
626 \\
646 \\
655 \\
665 \\
631\end{array}$ & $\begin{array}{l}370 \\
375 \\
395 \\
360 \\
380 \\
370 \\
370 \\
370 \\
425 \\
109 \\
405 \\
405 \\
395\end{array}$ & $\begin{array}{l}700 \\
710 \\
720 \\
725 \\
725 \\
720 \\
720 \\
730 \\
730 \\
730 \\
725 \\
735 \\
723\end{array}$ & $\begin{array}{l}248 \\
248 \\
240 \\
248 \\
248 \\
248 \\
248 \\
248 \\
248 \\
248 \\
248 \\
248 \\
248\end{array}$ & $\begin{array}{l}=- \\
=- \\
=- \\
=- \\
=- \\
= \\
= \\
= \\
= \\
=\end{array}$ & $\begin{array}{r}1,815 \\
1,900 \\
1,907 \\
1,849 \\
1,832 \\
1,642 \\
1,850 \\
1,850 \\
1,871 \\
1,840 \\
1,753 \\
21,872 \\
81,848\end{array}$ & $\begin{array}{l}1,095 \\
1,095 \\
1,105 \\
1,110 \\
1,110 \\
1,105 \\
1,105 \\
1,115 \\
1,115 \\
1,115 \\
1,110 \\
1,120 \\
1,108\end{array}$ & $\begin{array}{l}1,277 \\
1,287 \\
1,297 \\
1,302 \\
1,302 \\
1,297 \\
1,297 \\
1,307 \\
1,307 \\
1,307 \\
1,302 \\
1,317 \\
1,300\end{array}$ & $\begin{array}{l}1,455 \\
1,465 \\
1,475 \\
1,480 \\
1,480 \\
1,475 \\
1,475 \\
1,485 \\
1,485 \\
1,495 \\
1,480 \\
1,490 \\
1,478\end{array}$ & $\begin{array}{r}5,663 \\
5,728 \\
5,762 \\
5,634 \\
5,576 \\
5,555 \\
5,592 \\
5,616 \\
5,719 \\
5,709 \\
\mathbf{R 5}, 669 \\
5,807 \\
5,669\end{array}$ \\
\hline
\end{tabular}

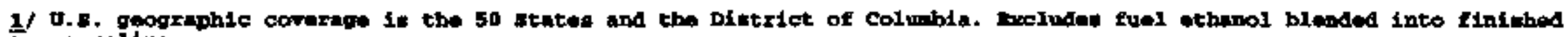
notor trasoline.

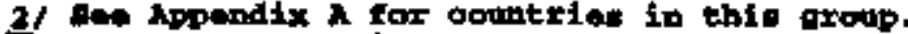

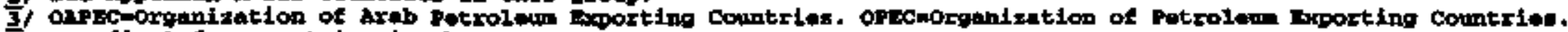

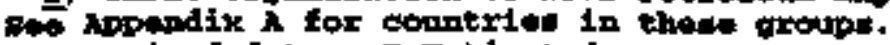

R=horised date. Bartionted.

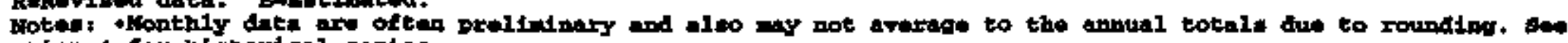

saction i for himtorioal inries.

soanton: too and of gection 1. 
Table 1.4 World OAl Bupply, 1985-PLeant

(Frounand Bextela per Din)

\begin{tabular}{|c|c|c|c|c|c|c|}
\hline & & $\begin{array}{l}\text { onited } \\
\text { statene }\end{array}$ & Porting & arpscy & OPXC3/ & World \\
\hline $\begin{array}{l}1985 \\
1966 \\
1987 \\
1988 \\
1999 \\
1990 \\
1991 \\
1992 \\
1993 \\
1994 \\
1995\end{array}$ & 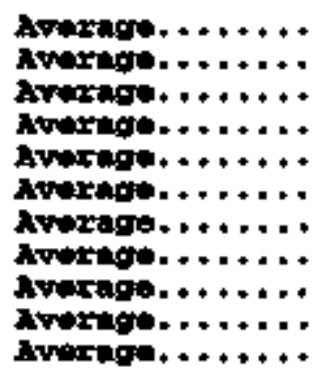 & $\begin{array}{r}11,192 \\
10,905 \\
10,640 \\
10,473 \\
9,800 \\
9,677 \\
9,683 \\
9,769 \\
9,602 \\
9,413 \\
9,400\end{array}$ & $\begin{array}{l}10,320 \\
12,411 \\
12,969 \\
14,310 \\
15,733 \\
18,249 \\
15,704 \\
17,010 \\
17,792 \\
18,072 \\
18,439\end{array}$ & $\begin{array}{l}11,498 \\
13,653 \\
13,992 \\
15,662 \\
16,658 \\
17,232 \\
16,747 \\
17,871 \\
18,505 \\
18,745 \\
19,192\end{array}$ & $\begin{array}{l}17,151 \\
19,310 \\
19,509 \\
21,465 \\
23,329 \\
24,536 \\
24,625 \\
25,818 \\
26,610 \\
27,031 \\
27,654\end{array}$ & $\begin{array}{l}59,264 \\
61,771 \\
62,429 \\
64,707 \\
65,894 \\
66,756 \\
66,634 \\
66,937 \\
67,345 \\
68,219 \\
69,916\end{array}$ \\
\hline 1996 & 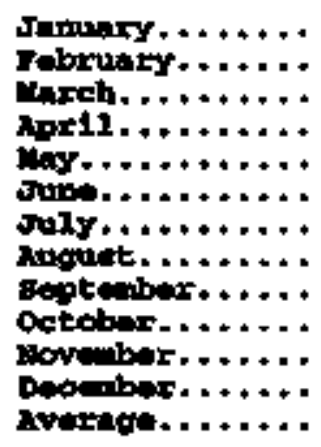 & $\begin{array}{l}9,346 \\
9,348 \\
9,503 \\
9,430 \\
9,357 \\
9,369 \\
9,328 \\
9,365 \\
9,516 \\
9,515 \\
9,636 \\
9,620 \\
9,445\end{array}$ & $\begin{array}{l}18,382 \\
18,467 \\
18,517 \\
18,297 \\
18,307 \\
18,477 \\
18,527 \\
18,452 \\
18,547 \\
18,507 \\
18,477 \\
18,977 \\
18,4 \$ 1\end{array}$ & $\begin{array}{l}19,059 \\
19,196 \\
19,207 \\
19,034 \\
19,104 \\
19,179 \\
19,279 \\
19,179 \\
19,254 \\
19,319 \\
19,259 \\
19,133 \\
19,243\end{array}$ & $\begin{array}{l}27,996 \\
28,101 \\
28,201 \\
27,561 \\
38,026 \\
28,171 \\
28,261 \\
28,276 \\
28,351 \\
28,451 \\
28,471 \\
29,036 \\
28,277\end{array}$ & $\begin{array}{l}71,040 \\
71,342 \\
71,413 \\
71,299 \\
71,236 \\
71,500 \\
71,690 \\
71,253 \\
71,741 \\
72,213 \\
72,766 \\
73,355 \\
71,738\end{array}$ \\
\hline 1997 & 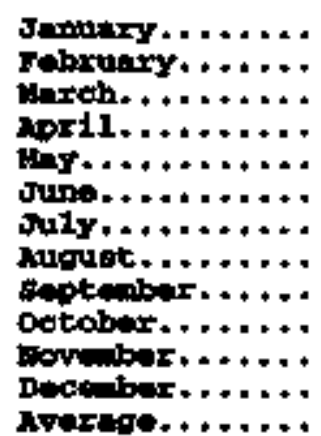 & $\begin{array}{r}9,249 \\
9,519 \\
9,482 \\
9,385 \\
9,321 \\
9,253 \\
9,407 \\
9,346 \\
9,503 \\
9,531 \\
99,458 \\
99,557 \\
0,417\end{array}$ & $\begin{array}{l}19,162 \\
19,377 \\
19,607 \\
19,762 \\
19,512 \\
19,122 \\
19,107 \\
20,127 \\
20,157 \\
20,197 \\
19,957 \\
19,573 \\
19,641\end{array}$ & $\begin{array}{l}10,889 \\
20,113 \\
20,363 \\
20,324 \\
20,334 \\
19,789 \\
19,849 \\
20,859 \\
21,079 \\
20,969 \\
20,679 \\
20,305 \\
20,397\end{array}$ & $\begin{array}{r}929,246 \\
29,521 \\
29,716 \\
29,936 \\
29,706 \\
829,316 \\
729,321 \\
30,481 \\
30,161 \\
30,601 \\
30,401 \\
30,0 \pm 7 \\
29,895\end{array}$ & 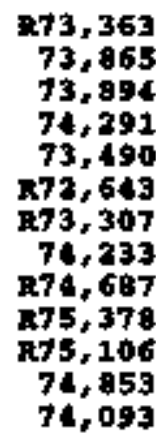 \\
\hline
\end{tabular}

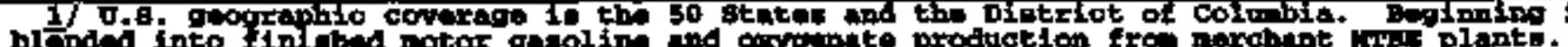

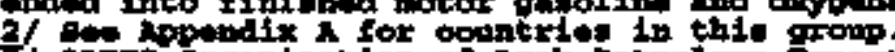

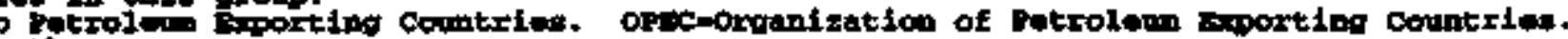

-

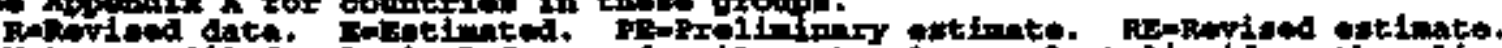

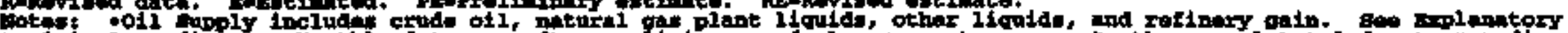

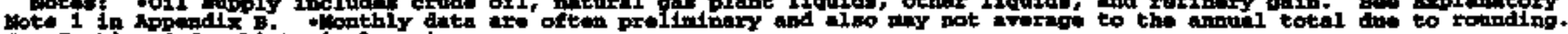

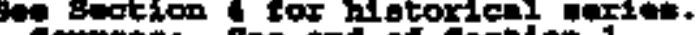

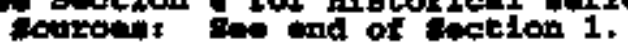




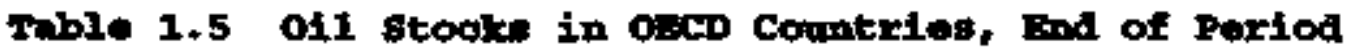
(rillion nerrels)

\begin{tabular}{|c|c|c|c|c|c|c|c|c|c|c|c|}
\hline & & Franct & Italy & Cexping $1 /$ & $\begin{array}{l}\text { tonited } \\
\text { xingdoen }\end{array}$ & $\begin{array}{c}\text { ORCD } \\
\text { Earoge } 2 /\end{array}$ & $\begin{array}{l}\text { United } \\
\text { gtatera } /\end{array}$ & Canada & Japan & Others & $\begin{array}{l}\text { Total } \\
\text { OBCD }\end{array}$ \\
\hline \multirow[t]{2}{*}{$\begin{array}{l}1985 \\
1986 \\
1987 \\
1988 \\
1969 \\
1990 \\
1991 \\
1992 \\
1993 \\
1994 \\
1995 \\
1996\end{array}$} & 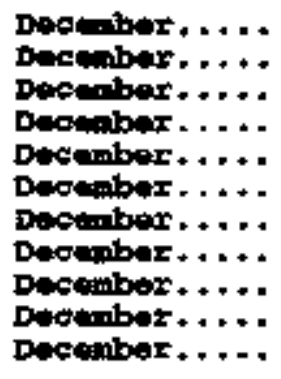 & $\begin{array}{l}139 \\
127 \\
127 \\
140 \\
130 \\
140 \\
153 \\
146 \\
158 \\
158 \\
159\end{array}$ & $\begin{array}{l}157 \\
155 \\
169 \\
155 \\
164 \\
172 \\
160 \\
174 \\
163 \\
164 \\
162\end{array}$ & $\begin{array}{l}233 \\
252 \\
259 \\
266 \\
271 \\
265 \\
288 \\
310 \\
309 \\
312 \\
301\end{array}$ & $\begin{array}{l}123 \\
124 \\
121 \\
112 \\
119 \\
112 \\
119 \\
113 \\
119 \\
115 \\
107\end{array}$ & $\begin{array}{l}1,092 \\
1,133 \\
1,130 \\
1,118 \\
1,133 \\
1,163 \\
1,181 \\
1,219 \\
1,221 \\
1,240 \\
1,228\end{array}$ & $\begin{array}{l}1,519 \\
1,593 \\
1,607 \\
1,597 \\
1,581 \\
1,621 \\
1,617 \\
1,592 \\
1,647 \\
1,653 \\
1,563\end{array}$ & $\begin{array}{l}113 \\
111 \\
126 \\
116 \\
114 \\
121 \\
119 \\
107 \\
105 \\
119 \\
109\end{array}$ & $\begin{array}{l}494 \\
509 \\
540 \\
530 \\
577 \\
590 \\
606 \\
603 \\
618 \\
645 \\
630\end{array}$ & $\begin{array}{l}66 \\
72 \\
71 \\
71 \\
71 \\
73 \\
65 \\
67 \\
69 \\
69 \\
71\end{array}$ & $\begin{array}{l}3,284 \\
3,418 \\
3,474 \\
3,440 \\
3,476 \\
3,568 \\
3,588 \\
3,589 \\
3,661 \\
3,726 \\
3,601\end{array}$ \\
\hline & 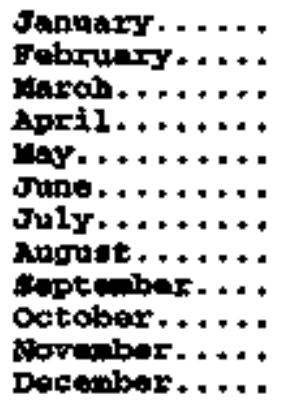 & $\begin{array}{l}154 \\
156 \\
156 \\
165 \\
163 \\
160 \\
162 \\
160 \\
152 \\
156 \\
160 \\
158\end{array}$ & $\begin{array}{l}157 \\
156 \\
153 \\
150 \\
157 \\
158 \\
155 \\
159 \\
162 \\
155 \\
152 \\
152\end{array}$ & $\begin{array}{l}301 \\
298 \\
296 \\
298 \\
295 \\
296 \\
297 \\
295 \\
295 \\
296 \\
297 \\
300\end{array}$ & $\begin{array}{l}107 \\
103 \\
106 \\
109 \\
105 \\
104 \\
105 \\
101 \\
105 \\
104 \\
106 \\
109\end{array}$ & $\begin{array}{l}1,236 \\
1,224 \\
1,212 \\
1,236 \\
1,233 \\
1,229 \\
1,242 \\
1,237 \\
1,229 \\
1,237 \\
1,243 \\
1,256\end{array}$ & $\begin{array}{l}1,544 \\
1,500 \\
1,482 \\
1,502 \\
1,520 \\
1,546 \\
1,550 \\
1,545 \\
1,551 \\
1,530 \\
1,522 \\
1,507\end{array}$ & $\begin{array}{l}104 \\
102 \\
109 \\
109 \\
107 \\
107 \\
110 \\
110 \\
113 \\
111 \\
105 \\
103\end{array}$ & $\begin{array}{l}638 \\
615 \\
627 \\
622 \\
641 \\
640 \\
637 \\
658 \\
664 \\
673 \\
665 \\
651\end{array}$ & $\begin{array}{l}79 \\
69 \\
70 \\
72 \\
75 \\
73 \\
83 \\
79 \\
83 \\
82 \\
81 \\
74\end{array}$ & $\begin{array}{l}3,596 \\
3,511 \\
3,500 \\
3,540 \\
3,575 \\
3,597 \\
3,621 \\
3,629 \\
3,641 \\
3,640 \\
3,616 \\
3,591\end{array}$ \\
\hline 1997 & 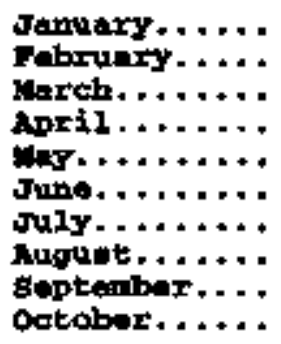 & $\begin{array}{l}156 \\
159 \\
160 \\
159 \\
163 \\
153 \\
153 \\
159 \\
157 \\
152\end{array}$ & $\begin{array}{l}158 \\
156 \\
160 \\
151 \\
150 \\
151 \\
150 \\
151 \\
144 \\
144\end{array}$ & $\begin{array}{l}304 \\
307 \\
311 \\
300 \\
309 \\
298 \\
304 \\
303 \\
291 \\
290\end{array}$ & $\begin{array}{l}107 \\
105 \\
109 \\
108 \\
109 \\
111 \\
106 \\
109 \\
106 \\
106\end{array}$ & $\begin{array}{r}1,279 \\
1,269 \\
1,272 \\
1,247 \\
1,246 \\
1,331 \\
1,227 \\
1,255 \\
1,290 \\
1,219\end{array}$ & $\begin{array}{l}1,503 \\
1,482 \\
2,512 \\
1,519 \\
1,562 \\
1,577 \\
1,559 \\
1,570 \\
1,594 \\
1,598\end{array}$ & $\begin{array}{l}104 \\
101 \\
105 \\
108 \\
104 \\
103 \\
105 \\
R 113 \\
109 \\
112\end{array}$ & $\begin{array}{l}650 \\
642 \\
650 \\
665 \\
664 \\
662 \\
670 \\
665 \\
684 \\
692\end{array}$ & $\begin{array}{l}84 \\
75 \\
76 \\
80 \\
81 \\
83 \\
81 \\
80 \\
77 \\
82\end{array}$ & $\begin{array}{r}3,616 \\
3,570 \\
3,616 \\
3,619 \\
3,657 \\
3,656 \\
\mathrm{R3}, 641 \\
\mathrm{R3}, 617 \\
\mathrm{R3}, 689 \\
3,713\end{array}$ \\
\hline
\end{tabular}

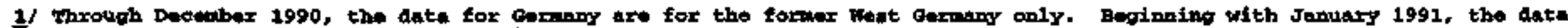

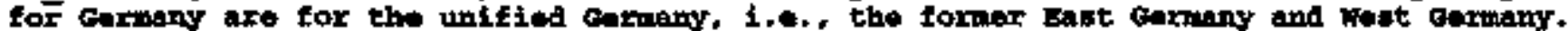

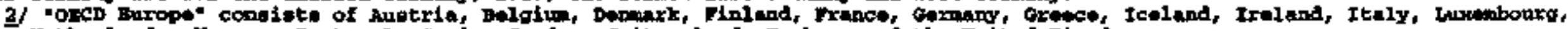

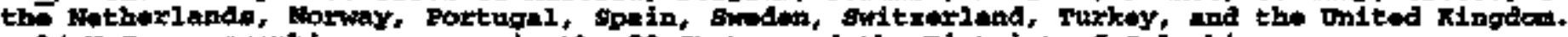

$3 /$ U.s. goosraphic coverage is the 50 states and the pistrict of columbla.

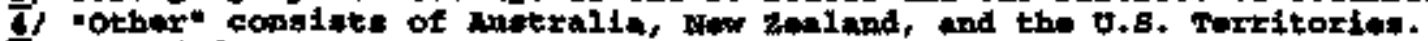

Reruriend dati.

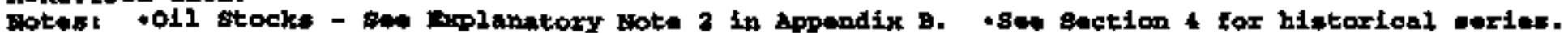

sourget: get and of stetion 1 . 


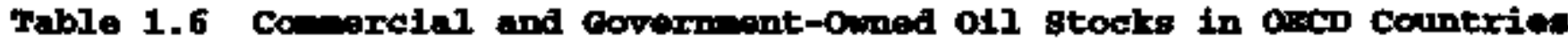 (aillios barrals)}

\begin{tabular}{|c|c|c|c|}
\hline Count:ry & Coungelent & corverinent & 2otel \\
\hline 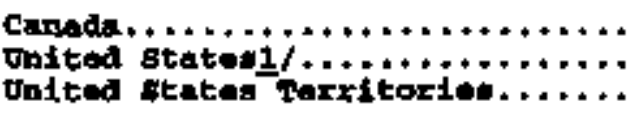 & $\begin{array}{r}109 \\
1.031 \\
27\end{array}$ & $\begin{array}{r}0 \\
563 \\
0\end{array}$ & $\begin{array}{r}109 \\
1,594 \\
27\end{array}$ \\
\hline 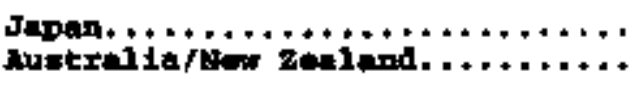 & $\begin{array}{r}360 \\
50\end{array}$ & $\begin{array}{r}312 \\
\mathbf{M A}\end{array}$ & $\begin{array}{r}680 \\
50\end{array}$ \\
\hline 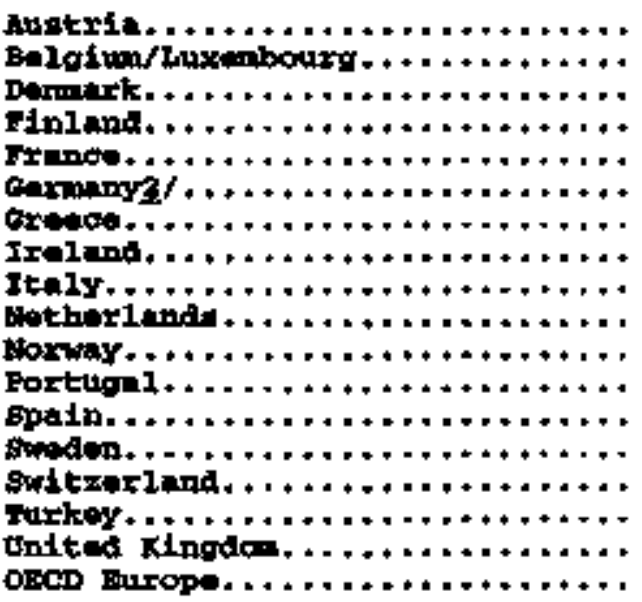 & $\begin{array}{r}22 \\
31 \\
24 \\
25 \\
157 \\
236 \\
27 \\
7 \\
130 \\
99 \\
49 \\
20 \\
100 \\
30 \\
46 \\
51 \\
106 \\
167\end{array}$ & $\begin{array}{r}0 \\
0 \\
0 \\
0 \\
0 \\
5 \\
10 \\
2 \\
6 \\
0 \\
0 \\
0 \\
0 \\
0 \\
0 \\
0 \\
0 \\
63\end{array}$ & $\begin{array}{r}22 \\
31 \\
24 \\
25 \\
157 \\
291 \\
27 \\
9 \\
144 \\
99 \\
49 \\
20 \\
100 \\
30 \\
46 \\
51 \\
106 \\
1,230\end{array}$ \\
\hline Total ored $\ldots \ldots \ldots \ldots \ldots \ldots \ldots \ldots \ldots$ & 2,791 & 938 & 3,689 \\
\hline
\end{tabular}

IJ J.s. geodriphic oovertege id the 50 states and the Diptribt of colubia.

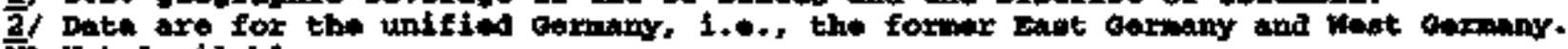
Whot Arillable.

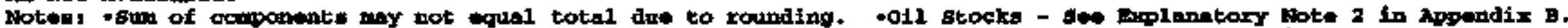

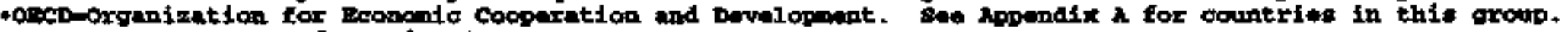
sourosi geo and of geotion 1 . 
Table 1.7. CacD ofl De-ina, 1985-Provent (thowoand Barzele par Day)

\begin{tabular}{|c|c|c|c|c|c|c|c|c|c|c|c|}
\hline & & Exthes & Itanly & owrongyt & $\begin{array}{l}\text { Dinited } \\
\text { Kingilo }\end{array}$ & Oixcoped & $\begin{array}{l}\text { Uaited } \\
\text { gtatens/ }\end{array}$ & Conndin & Japin & Otbitst & $\begin{array}{l}\text { toten } \\
\text { OTCD }\end{array}$ \\
\hline $\begin{array}{l}1985 \\
1996 \\
1997 \\
1988 \\
1989 \\
1990 \\
1991 \\
1992 \\
1993 \\
1994 \\
1995\end{array}$ & 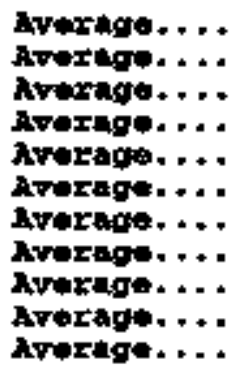 & $\begin{array}{l}1,775 \\
1,772 \\
1,789 \\
1,797 \\
1,857 \\
1,818 \\
1,935 \\
1,926 \\
1,875 \\
1,833 \\
1,896\end{array}$ & $\begin{array}{l}1,717 \\
1,738 \\
1,853 \\
1,836 \\
1,930 \\
1,872 \\
1,863 \\
1,937 \\
1,852 \\
1,841 \\
2,048\end{array}$ & $\begin{array}{l}2,338 \\
2,498 \\
2,424 \\
2,427 \\
2,280 \\
2,392 \\
2,838 \\
2,843 \\
2,900 \\
2,879 \\
2,875\end{array}$ & $\begin{array}{l}1,634 \\
1,649 \\
1,603 \\
1,697 \\
1,739 \\
1,752 \\
1,801 \\
1,803 \\
1,815 \\
1,837 \\
1,845\end{array}$ & $\begin{array}{l}11,681 \\
12,102 \\
12,255 \\
12,427 \\
12,531 \\
12,629 \\
13,391 \\
13,605 \\
13,523 \\
13,597 \\
24,120\end{array}$ & $\begin{array}{l}15,736 \\
16,281 \\
16,665 \\
17,283 \\
17,325 \\
16,988 \\
16,714 \\
17,033 \\
17,237 \\
17,710 \\
17,725\end{array}$ & $\begin{array}{l}1,504 \\
1,506 \\
1,548 \\
1,693 \\
1,733 \\
1,690 \\
1,622 \\
1,643 \\
1,698 \\
1,727 \\
1,755\end{array}$ & $\begin{array}{l}4,394 \\
4,439 \\
4,484 \\
1,752 \\
4,983 \\
5,140 \\
5,284 \\
5,446 \\
5,401 \\
5,674 \\
5,711\end{array}$ & $\begin{array}{r}976 \\
951 \\
959 \\
939 \\
999 \\
1,027 \\
1,056 \\
1,041 \\
1,118 \\
1,174 \\
1,227\end{array}$ & $\begin{array}{l}34,271 \\
35,279 \\
35,911 \\
37,093 \\
37,570 \\
37,475 \\
38,067 \\
38,769 \\
38,967 \\
39,890 \\
40,537\end{array}$ \\
\hline & 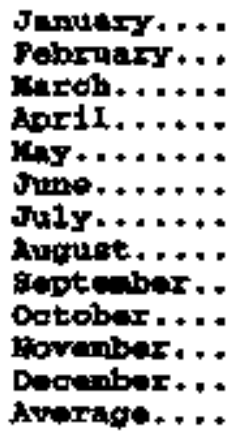 & $\begin{array}{l}1,879 \\
2,183 \\
1,979 \\
1,919 \\
1,810 \\
1,819 \\
1,977 \\
1,841 \\
1,929 \\
1,989 \\
1,880 \\
2,021 \\
1,935\end{array}$ & $\begin{array}{l}2,113 \\
2,259 \\
2,189 \\
1,961 \\
1,080 \\
1,908 \\
2,158 \\
1,786 \\
2,074 \\
2,201 \\
2,083 \\
2,088 \\
2,058\end{array}$ & $\begin{array}{l}2,901 \\
3,030 \\
2,860 \\
2,743 \\
2,864 \\
2,030 \\
2,957 \\
3,035 \\
3,095 \\
2,060 \\
2,975 \\
2,796 \\
2,911\end{array}$ & $\begin{array}{l}1,762 \\
1,919 \\
1,959 \\
1,853 \\
1,846 \\
1,738 \\
1,790 \\
1,795 \\
1,877 \\
1,910 \\
1,966 \\
1,836 \\
1,845\end{array}$ & $\begin{array}{l}14,036 \\
15,138 \\
14,273 \\
13,676 \\
13,778 \\
13,597 \\
1,245 \\
13,873 \\
14,775 \\
14,722 \\
14,700 \\
14,458 \\
14,269\end{array}$ & $\begin{array}{l}18,261 \\
18,620 \\
18,301 \\
17,885 \\
17,957 \\
18,107 \\
18,211 \\
18,650 \\
17,655 \\
19,171 \\
18,535 \\
18,334 \\
18,309\end{array}$ & $\begin{array}{l}1,805 \\
1,874 \\
1,704 \\
1,667 \\
1,715 \\
1,796 \\
1,802 \\
1,880 \\
1,763 \\
1,809 \\
1,941 \\
1,771 \\
1,797\end{array}$ & $\begin{array}{l}6,328 \\
6,886 \\
6,437 \\
5,748 \\
5,147 \\
5,114 \\
5,502 \\
5,567 \\
5,361 \\
5,580 \\
6,114 \\
6,618 \\
5,867\end{array}$ & $\begin{array}{l}1,165 \\
1,172 \\
1,151 \\
1,154 \\
1,113 \\
1,128 \\
1,084 \\
1,123 \\
1,024 \\
1,133 \\
1,064 \\
1,197 \\
1,124\end{array}$ & $\begin{array}{l}41,595 \\
43,690 \\
41,907 \\
40,130 \\
39,709 \\
39,742 \\
40,843 \\
41,091 \\
40,579 \\
42,414 \\
42,354 \\
42,402 \\
41,366\end{array}$ \\
\hline & 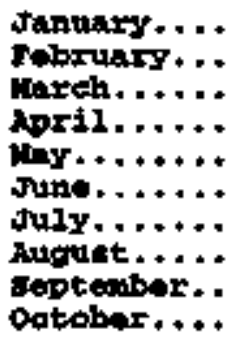 & $\begin{array}{r}2,165 \\
2,137 \\
1,796 \\
1,910 \\
1,707 \\
1,882 \\
2,068 \\
21,787 \\
\mathrm{RI}, 990 \\
2,146\end{array}$ & $\begin{array}{r}2,037 \\
2,126 \\
1,928 \\
1,999 \\
1,898 \\
1,948 \\
2,029 \\
21,809 \\
\mathrm{R2}, 181 \\
2,216\end{array}$ & $\begin{array}{r}2,901 \\
2,673 \\
2,685 \\
3,225 \\
2,787 \\
3,136 \\
13,098 \\
\mathrm{R2}, 740 \\
\mathrm{R3}, 161 \\
2,977\end{array}$ & $\begin{array}{r}1,828 \\
1,907 \\
1,776 \\
1,829 \\
1,711 \\
1,791 \\
71,766 \\
1,724 \\
1,833 \\
1,859\end{array}$ & $\begin{array}{r}14,633 \\
14,580 \\
13,579 \\
214,664 \\
R 13,510 \\
R 1,, 316 \\
\mathrm{R} 14,727 \\
\mathrm{R} 13,491 \\
\mathrm{RI}, 974 \\
15,014\end{array}$ & $\begin{array}{l}18,560 \\
18,305 \\
17,869 \\
18,572 \\
18,244 \\
18,563 \\
19,065 \\
18,506 \\
18,480 \\
19,121\end{array}$ & $\begin{array}{r}1,862 \\
1,862 \\
1,780 \\
1,715 \\
1,923 \\
1,913 \\
21,949 \\
1,912 \\
11,057 \\
1,846\end{array}$ & $\begin{array}{r}6,291 \\
6,751 \\
6,146 \\
5,303 \\
5,076 \\
5,131 \\
15,440 \\
15,391 \\
\mathrm{R5}, 411 \\
5,450\end{array}$ & $\begin{array}{r}1,138 \\
1,140 \\
1,141 \\
1,174 \\
1,065 \\
1,090 \\
\mathrm{R1}, 142 \\
\mathrm{R1}, 106 \\
\mathrm{R} 1,158 \\
1,146\end{array}$ & $\begin{array}{r}42,483 \\
42,641 \\
40,515 \\
\mathrm{R} 41,457 \\
\mathrm{R39}, 719 \\
\mathrm{R} 41,013 \\
\mathrm{t} 42,323 \\
\mathrm{R} 40,406 \\
\mathrm{R41}, 860 \\
42,57 \mathrm{~s}\end{array}$ \\
\hline
\end{tabular}

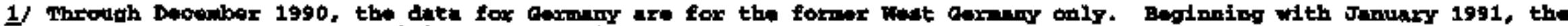

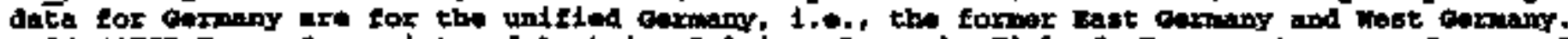

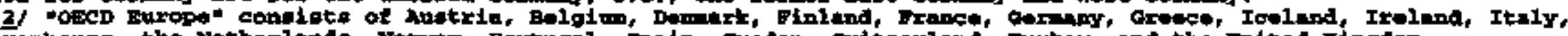

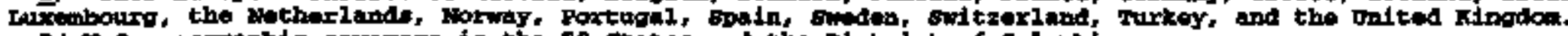

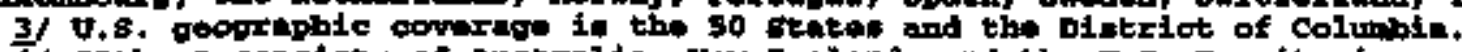

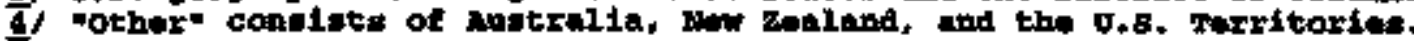

$\overline{\mathrm{R}}=\mathrm{R}$ erirod ante.

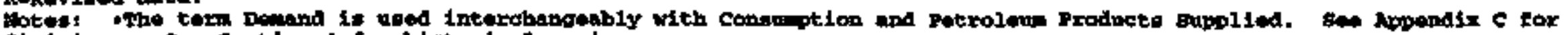

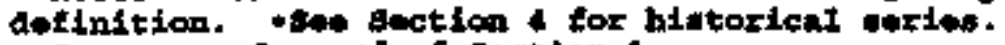

Bourdes! foe and of soction 1 . 


\section{Sources for Section 1 (Annual and Monthly Oil Data)}

Crude Oil Production, Natural Gas Liquids Production, Other Liquids, and Retinery Gain

United States: Esergy Information Administration (ElA), Petroleum Supply Animis 199 and Peirokum Supply Monthly, Januany 1998.

Othor Countries: Annal data - EA, luternational Enesgy Annuad, various issues. Monthly data - Canada -- Maclean Humer Pubtishing Company, Oilweek, various issues. Dentmark, Mexico, Netherlands, Norway, Germany, and all OPEC countries - Petroleum and Energy Inteligence Wedty. Int. Petroleum Inlelligence Weckly, varions isues. All Other Countries - PennWell Publishing Company, Oil and Gas lournal, various issues, Refinery Gains; National Peuroleum Council, U.S. Poroleum Refinery Study Octaber 1986.

\section{Oil Stocks}

United States: EIA, Petroleum Supply Anmual 1996 and Petroleum Surpily Monthly, January 1998.

Other OECD Countries: • Organization for Economic Cooperation and Development (OECD), Oumaketly Oil Statislics ard Energy Balances, various issues. OECD, Monthly OAl Siatistics data base, 1985-1997.

\section{Oil Demand}

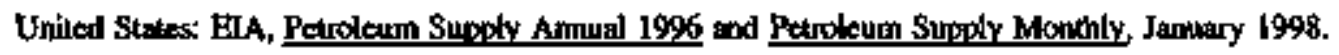

Other OECD Cowntries: * OECD, Ouarterly Oil Statistics and Enerxy Balangen, various issues. - OECD, Monaly Oil Stalistics data baso, 1985-1997. 

2. International Oll Balance Data 
Table 2.1 Worla ofl Balance, 1993-1997

(atllion Barrela Dar Dar)

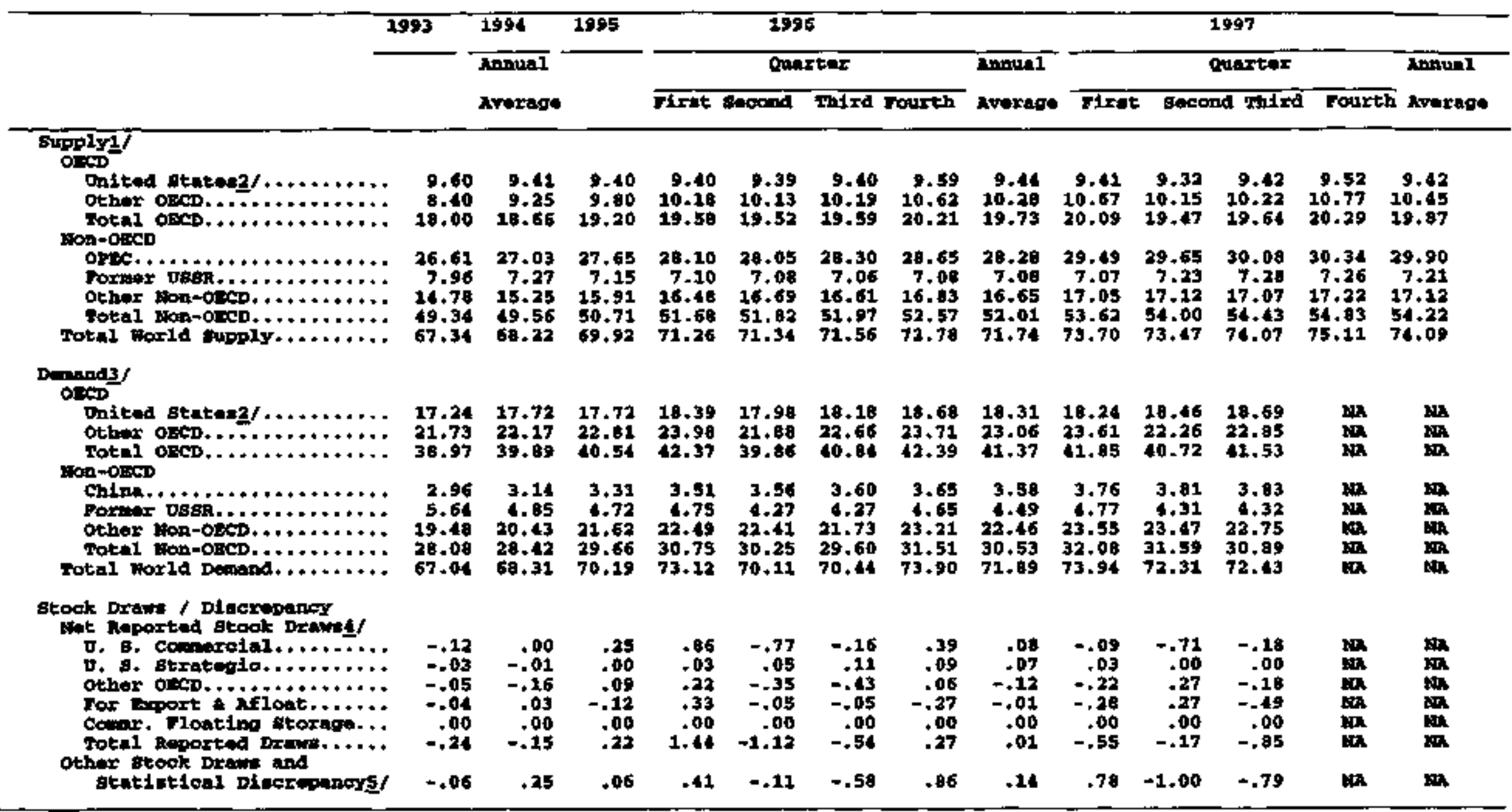

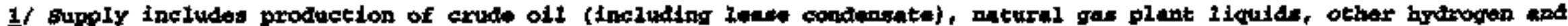

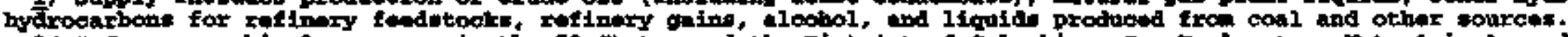

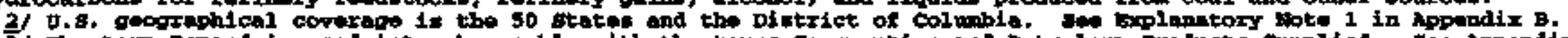

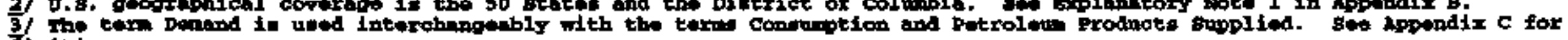
alfiattion.

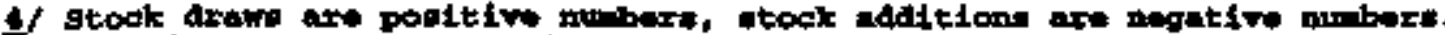

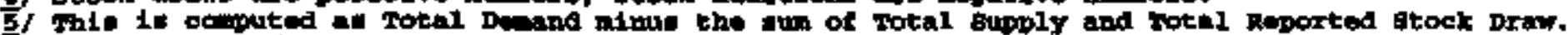

bis = tot Arailable.

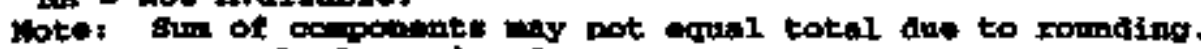

gourcert for and of Fection 2. 


\begin{tabular}{|c|c|c|c|c|c|c|c|c|c|c|c|c|c|}
\hline & & \multirow{3}{*}{ 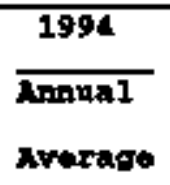 } & \multirow[t]{3}{*}{1995} & \multicolumn{5}{|c|}{1996} & \multicolumn{4}{|c|}{1997} & \multirow{3}{*}{$\begin{array}{l}\overline{\text { Annual }} \\
\text { Arorags }\end{array}$} \\
\hline & & & & \multicolumn{4}{|c|}{ Quicter } & \multirow{2}{*}{$\begin{array}{l}\text { Antal } \\
\text { Rintrage }\end{array}$} & \multicolumn{4}{|c|}{ Ounxter } & \\
\hline & & & & Firut & Beocind & Whited & Fourth & & Firtet & stoond & Ihitad & Fourth & \\
\hline 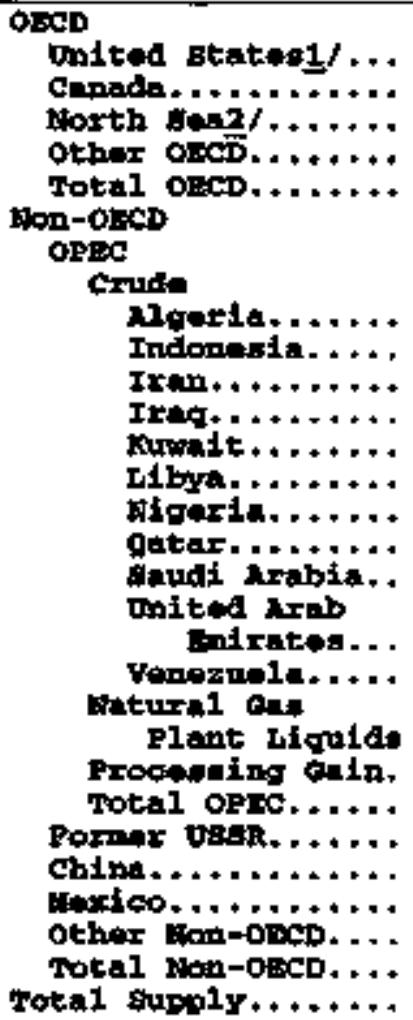 & $\begin{array}{r}1.16 \\
1.51 \\
3.54 \\
.51 \\
1.85 \\
1.36 \\
1.96 \\
. .41 \\
9.20 \\
2.16 \\
2.45 \\
1.44 \\
2.06 \\
7.96 \\
2.89 \\
3.16 \\
9.73 \\
49.34 \\
67.34\end{array}$ & $\begin{array}{r}1.18 \\
1.51 \\
3.67 \\
.53 \\
2.02 \\
1.38 \\
1.93 \\
.41 \\
6.12 \\
2.19 \\
2.59 \\
1.46 \\
.06 \\
27.03 \\
7.27 \\
2.94 \\
3.17 \\
9.14 \\
49.56 \\
68.22\end{array}$ & $\begin{array}{r}1.20 \\
1.50 \\
3.64 \\
.96 \\
2.06 \\
1.39 \\
1.99 \\
.48 \\
8.29 \\
2.28 \\
2.73 \\
1.51 \\
.06 \\
27.65 \\
7.15 \\
2.99 \\
3.09 \\
9.83 \\
50.71 \\
69.92\end{array}$ & $\begin{array}{r}1.22 \\
1.54 \\
3.71 \\
.55 \\
2.05 \\
1.10 \\
2.18 \\
.50 \\
6.20 \\
2.28 \\
2.96 \\
1.45 \\
.06 \\
28.10 \\
7.10 \\
3.09 \\
3.32 \\
10.07 \\
51.69 \\
71.26\end{array}$ & $\begin{array}{r}1.24 \\
1.54 \\
3.67 \\
.55 \\
2.06 \\
1.40 \\
2.19 \\
.50 \\
8.14 \\
2.27 \\
2.99 \\
1.45 \\
.06 \\
28.05 \\
7.09 \\
3.14 \\
3.38 \\
10.17 \\
51.82 \\
71.34\end{array}$ & $\begin{array}{r}1.25 \\
1.54 \\
3.71 \\
.55 \\
3.06 \\
1.40 \\
2.17 \\
.51 \\
8.24 \\
2.28 \\
3.07 \\
1.46 \\
.06 \\
28.30 \\
7.06 \\
3.14 \\
3.25 \\
10.27 \\
51.97 \\
71.56\end{array}$ & $\begin{array}{r}1.26 \\
1.57 \\
3.65 \\
.67 \\
2.09 \\
1.40 \\
2.22 \\
.53 \\
8.29 \\
2.29 \\
3.19 \\
1.45 \\
.06 \\
28.65 \\
7.09 \\
3.16 \\
3.27 \\
10.41 \\
52.57 \\
72.78\end{array}$ & $\begin{array}{r}1.24 \\
1.55 \\
1.69 \\
.59 \\
2.06 \\
1.40 \\
2.19 \\
.51 \\
0.22 \\
2.28 \\
3.05 \\
1.45 \\
.06 \\
28.28 \\
7.09 \\
3.13 \\
3.31 \\
10.22 \\
52.01 \\
71.74\end{array}$ & $\begin{array}{r}1.27 \\
1.59 \\
3.68 \\
1.13 \\
2.09 \\
1.43 \\
0.28 \\
. .58 \\
9.40 \\
2.33 \\
3.19 \\
1.46 \\
. .06 \\
29.49 \\
7.07 \\
3.27 \\
3.36 \\
10.47 \\
53.62 \\
73.70\end{array}$ & $\begin{array}{r}1.27 \\
1.56 \\
3.68 \\
1.07 \\
3.06 \\
1.45 \\
3.31 \\
.63 \\
8.55 \\
2.30 \\
3.24 \\
1.48 \\
29.06 \\
7.65 \\
3.23 \\
3.38 \\
10.50 \\
54.00 \\
73.47\end{array}$ & $\begin{array}{r}1.28 \\
1.52 \\
3.62 \\
1.28 \\
2.07 \\
1.45 \\
2.33 \\
. .68 \\
8.63 \\
2.32 \\
3.36 \\
1.48 \\
30.06 \\
7.28 \\
3.79 \\
3.49 \\
20.39 \\
54.43 \\
74.07\end{array}$ & $\begin{array}{r}1.28 \\
1.52 \\
3.67 \\
1.26 \\
2.11 \\
1.45 \\
2.36 \\
+74 \\
8.67 \\
2.31 \\
3.46 \\
1.49 \\
30.05 \\
7.26 \\
3.15 \\
3.52 \\
10.55 \\
56.63 \\
75.11\end{array}$ & $\begin{array}{r}1.29 \\
1.55 \\
3.66 \\
1.19 \\
2.09 \\
1.45 \\
2.32 \\
0.65 \\
2.34 \\
3.31 \\
1.49 \\
.06 \\
29.90 \\
7.21 \\
3.20 \\
3.44 \\
10.48 \\
54.22 \\
74.09\end{array}$ \\
\hline
\end{tabular}

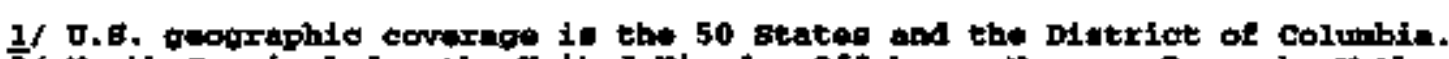

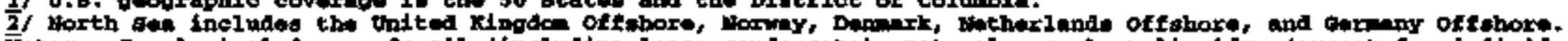

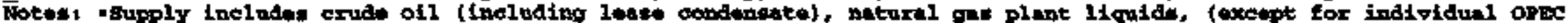

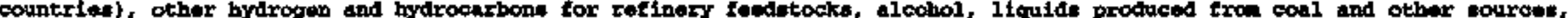

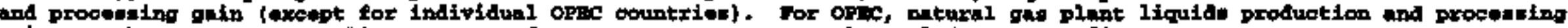

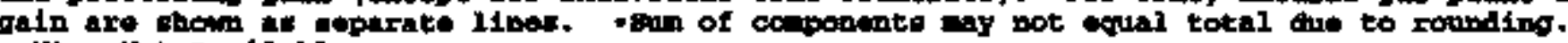

Wh Dot Avisiable.

sources: sec ond of gection 2 . 


\begin{tabular}{|c|c|c|c|c|c|c|c|c|c|c|c|}
\hline & \multirow[t]{3}{*}{1993} & 1994 & \multirow[t]{3}{*}{1995} & \multicolumn{4}{|c|}{1996} & \multicolumn{4}{|c|}{1997} \\
\hline & & \multirow[t]{2}{*}{ Iear ind } & & \multicolumn{4}{|c|}{ ouarter } & \multicolumn{4}{|c|}{ tuarteñ } \\
\hline & & & & $\overline{\text { Brfut }}$ & sacond & Thixd & Fourth & Plxat & Becond & mira & Fonsth \\
\hline 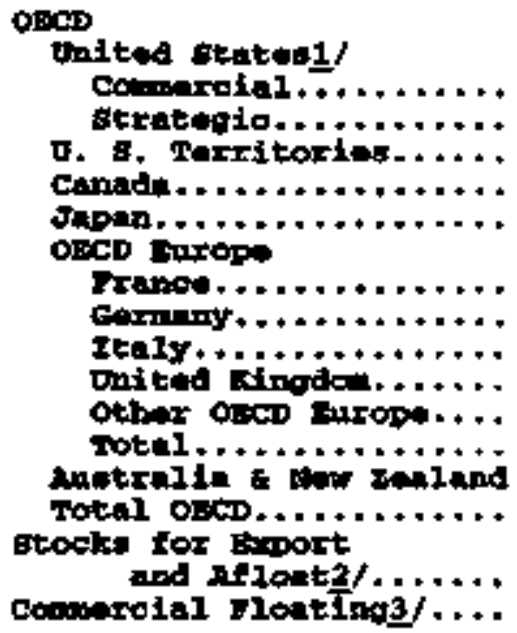 & $\begin{array}{r}1,060 \\
587 \\
25 \\
105 \\
618 \\
159 \\
309 \\
163 \\
118 \\
475 \\
1,221 \\
3,661 \\
\\
945 \\
70\end{array}$ & $\begin{array}{r}1,061 \\
592 \\
26 \\
119 \\
645 \\
158 \\
312 \\
164 \\
115 \\
490 \\
1,240 \\
4.726 \\
935 \\
70\end{array}$ & $\begin{array}{r}971 \\
592 \\
24 \\
109 \\
630 \\
\\
159 \\
301 \\
262 \\
107 \\
499 \\
1,228 \\
477 \\
3,601 \\
980 \\
70\end{array}$ & $\begin{array}{r}893 \\
589 \\
22 \\
109 \\
627 \\
156 \\
296 \\
153 \\
106 \\
501 \\
1,212 \\
4,500 \\
950 \\
70\end{array}$ & $\begin{array}{r}962 \\
584 \\
23 \\
107 \\
640 \\
160 \\
296 \\
158 \\
104 \\
511 \\
1,229 \\
50 \\
3,597 \\
953 \\
70\end{array}$ & $\begin{array}{r}977 \\
574 \\
29 \\
119 \\
664 \\
152 \\
295 \\
162 \\
105 \\
515 \\
1,229 \\
54 \\
3,641 \\
960 \\
70\end{array}$ & $\begin{array}{r}941 \\
566 \\
75 \\
103 \\
651 \\
\\
158 \\
300 \\
152 \\
108 \\
537 \\
1,356 \\
49 \\
3,591 \\
\\
995 \\
70\end{array}$ & $\begin{array}{r}949 \\
563 \\
23 \\
105 \\
650 \\
\\
160 \\
311 \\
260 \\
109 \\
532 \\
1,272 \\
53 \\
3,616 \\
1,010 \\
70\end{array}$ & $\begin{array}{r}1,014 \\
563 \\
27 \\
103 \\
662 \\
\\
153 \\
298 \\
151 \\
111 \\
518 \\
1,231 \\
56 \\
3,656 \\
965 \\
70\end{array}$ & $\begin{array}{r}1,031 \\
563 \\
27 \\
109 \\
690 \\
157 \\
291 \\
141 \\
106 \\
531 \\
1,230 \\
5,699 \\
3,090 \\
1,030 \\
70\end{array}$ & 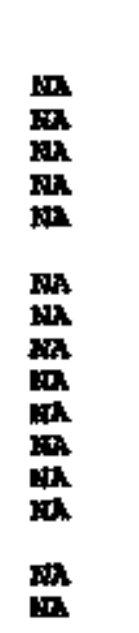 \\
\hline
\end{tabular}

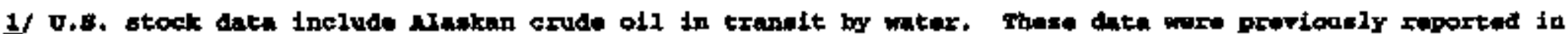

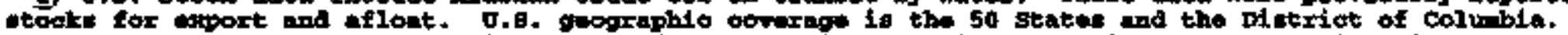

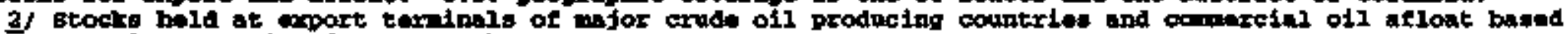

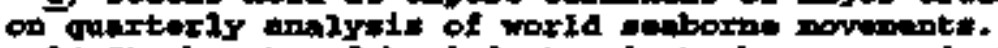

$3 /$ Btocks otored by industry in tankers wored oftebore. Bxcluded atrategle atorige.

Wa = lot Available.

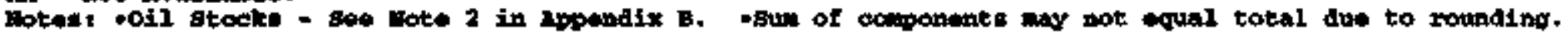

Bouroes: toe end of enction 3 . 
Table 2.4 World ofl Dwand, 1993-1997

(Millop Ecrols per bay)

\begin{tabular}{|c|c|c|c|c|c|c|c|c|c|}
\hline $199 \overline{3}$ & 1994 & & 1996 & & & & 1997 & & \\
\hline & Ammual & Qua & $\operatorname{tex}$ & Mnmual & & ous & Ear & & Anoulat \\
\hline & Averagt & First bocond & Third Bourteh & lovrate & pirat & seoond & Taird & Fonyth & Averapo \\
\hline
\end{tabular}

OEXP

\begin{tabular}{|c|c|c|c|c|c|c|c|c|c|c|c|c|c|}
\hline 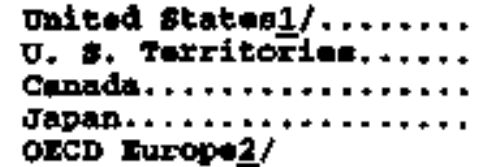 & $\begin{array}{r}17.24 \\
.24 \\
5.69\end{array}$ & $\begin{array}{r}17.72 \\
.26 \\
5.73 \\
5.67\end{array}$ & $\begin{array}{r}17.72 \\
.24 \\
1.76 \\
5.71\end{array}$ & $\begin{array}{r}18.99 \\
.21 \\
1.91 \\
6.54\end{array}$ & $\begin{array}{r}17.98 \\
.19 \\
2.73 \\
5.39\end{array}$ & $\begin{array}{r}18.18 \\
.17 \\
1.82 \\
5.48\end{array}$ & $\begin{array}{r}18.68 \\
.19 \\
1.84 \\
6.11\end{array}$ & $\begin{array}{r}18.31 \\
.19 \\
1.80 \\
5.87\end{array}$ & $\begin{array}{r}18.24 \\
.20 \\
1.83 \\
6.38\end{array}$ & $\begin{array}{r}18.46 \\
.17 \\
1.83 \\
5.17\end{array}$ & $\begin{array}{r}18.69 \\
.19 \\
1.91 \\
5.41\end{array}$ & $\begin{array}{l}\mathbf{n} \\
\mathbf{w n} \\
\mathbf{m}\end{array}$ & $\underset{m}{\min }$ \\
\hline 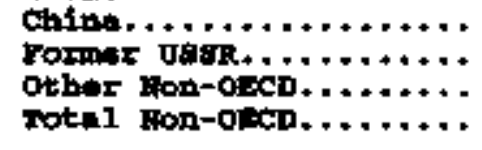 & $\begin{array}{r}2.96 \\
5.64 \\
19.48 \\
20.48\end{array}$ & $\begin{array}{r}3.14 \\
4.85 \\
20.43 \\
28 \cdot 42\end{array}$ & $\begin{array}{r}3.31 \\
4.72 \\
21.62 \\
29.66\end{array}$ & $\begin{array}{r}3.51 \\
4.75 \\
22.49 \\
30.75\end{array}$ & $\begin{array}{r}3.56 \\
4.27 \\
32.41 \\
30.25\end{array}$ & $\begin{array}{r}3.60 \\
4.27 \\
21.73 \\
29.60\end{array}$ & $\begin{array}{r}3.65 \\
1.65 \\
23.21 \\
31.51\end{array}$ & $\begin{array}{r}3.58 \\
4.49 \\
22.46 \\
30.59\end{array}$ & $\begin{array}{r}3.76 \\
4.77 \\
23.55 \\
32.08\end{array}$ & $\begin{array}{r}3.91 \\
4.91 \\
23.47 \\
31.59\end{array}$ & $\begin{array}{r}3.93 \\
4.92 \\
22.75 \\
30.89\end{array}$ & $\begin{array}{l}\text { wh } \\
\text { wh } \\
\text { wh }\end{array}$ & $\begin{array}{l}\text { Wh } \\
\text { WR } \\
\text { wo }\end{array}$ \\
\hline ottal & 67.04 & 68.31 & 70.19 & 73.12 & 70.11 & 70.44 & 73.90 & 71.89 & 33.94 & 72.31 & 72.43 & $\sin$ & $\mathbf{x}$ \\
\hline
\end{tabular}

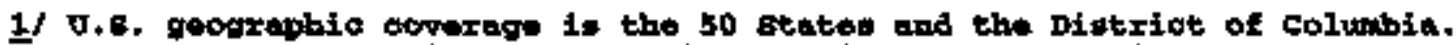

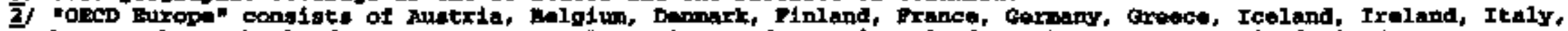

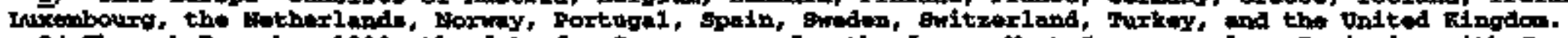

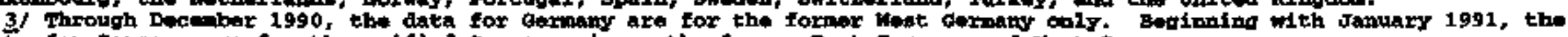

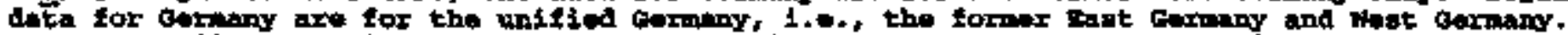

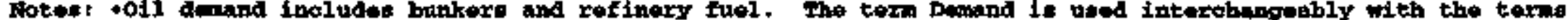

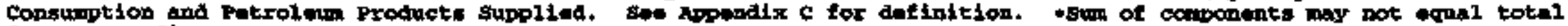
due to rounding.

in $=$ lot available.

souresa: tore ind of Bection 2. 


\section{Sonrces for Section 2 (International Oil Balance Data)}

Crude Oil Protuction, Natural Gas Liquids Prodtacion, Other Liquids, and Refincry Gain

United States: Energy Infornation Adminisurabion (EIA), Petrodeun Supgly Anpual 1996 and Petroleum Supply Monthly. January 1998.

Other Countries: Ammual daa - EIA, Lntemational Energy Anmual, various issues. Monthly data -- Canada - Maclean Hunter Publishing Company, Oilweek, various issues, Demmark, Mexicn, Netherlands, Norway, Germany, and all OPEC couniries - Peumbeum and Energy Intelligence Weekly. Inc., Petroleum Int-ilizence Weekly, yarious issues. Other Countries -- PennWel1 Publishing Company, Oil and Gas loumal, various issues. Refinery Gains: National Petroleum Council, U.S. Petroleum Refinery Siudy, October 1986.

Oil Stocks

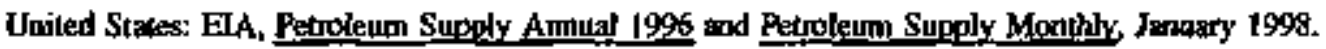

Other OECD Countries: - Organization for Economic Cooperation and Development (OBCD). Quanterly Oil Slatistics and Eaeray Balances, various isstes. - OECD, Monthly of Statistics dala base, 1990-1997. Siocks for Export and Afloat and Commertal Floating Stucks - Petroleum Exomonics Limiled, Gil Indestry Developonents, variues isspes.

Dil Dernand

United Statos: EIA, Peiroteum Supply Anmial 1996 and Peimolenm Supply Monthly, January 1998.

Other OECD Coumtries: + OECD. OEarterly Oil Stristics and Energy Balances, various issues. • OBCD, Monthly Oil Statistics data base, $1990-1997$.

OPEC Countries: ElA, Intemational Energy Ammual, various issues and Shent-Tem Energy Ondook, various issues.

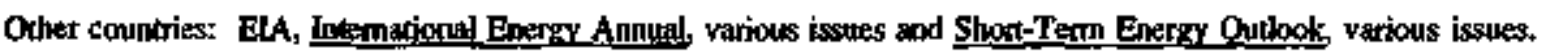


3. International Oil Imports 
Table 3.1 Avernge ret od1 Imports into ODeO Combries, (1996 Average)

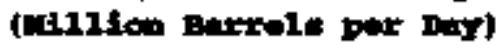

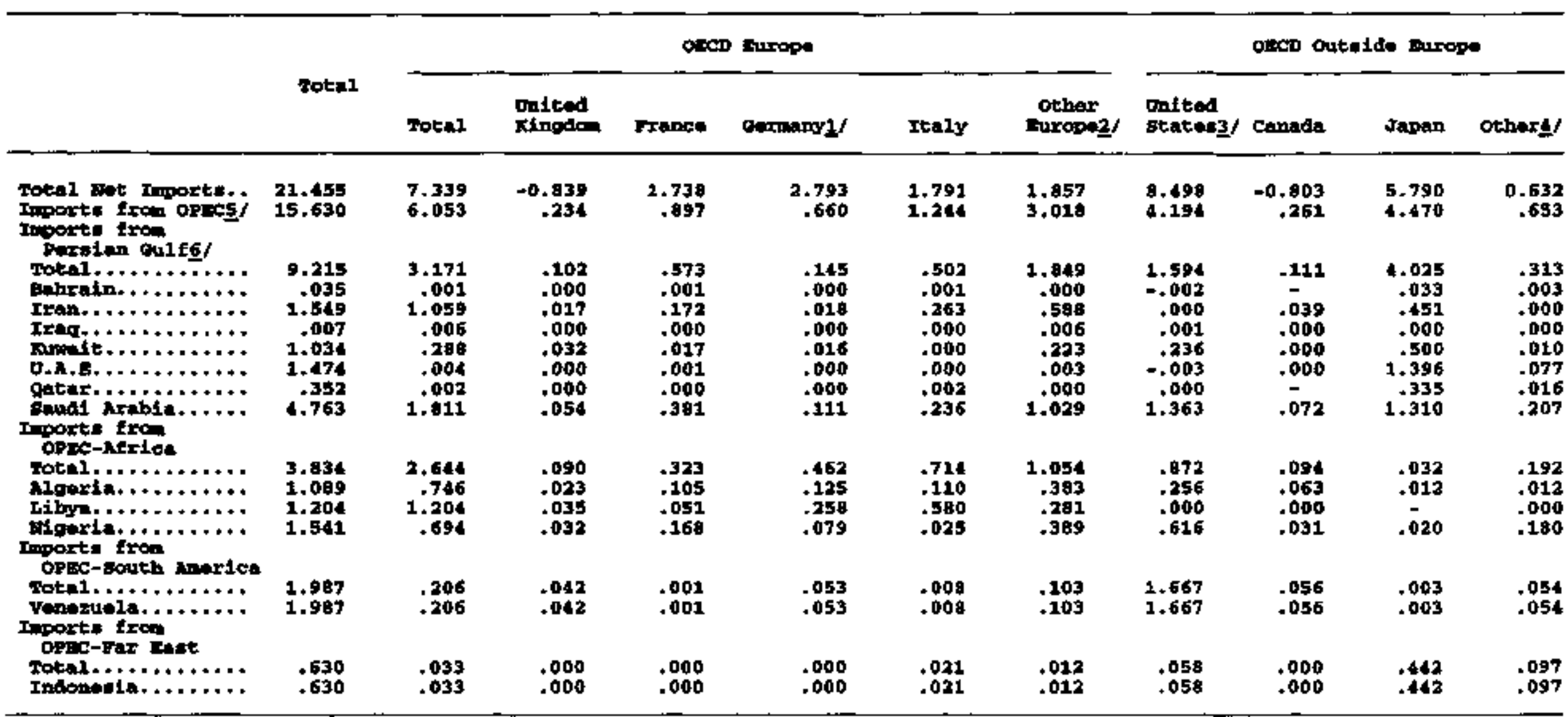

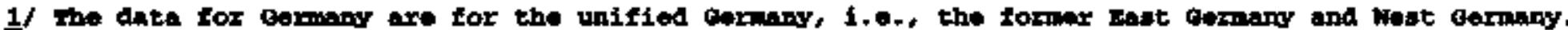

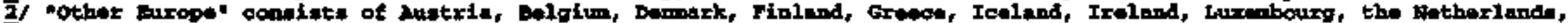

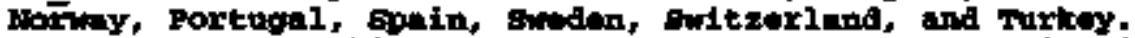

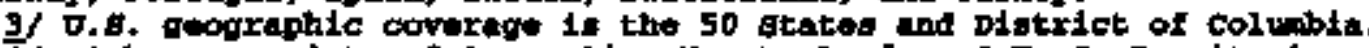

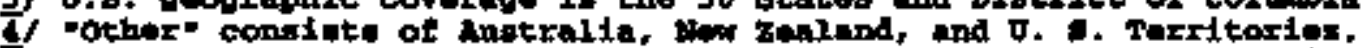

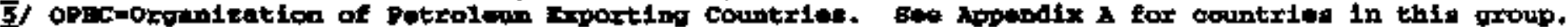

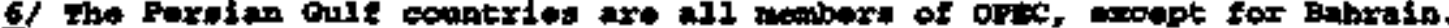

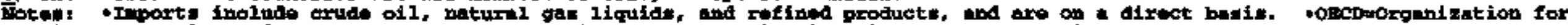

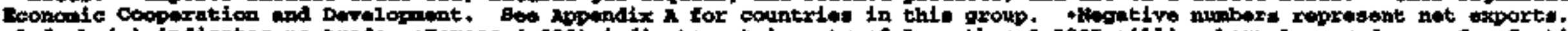

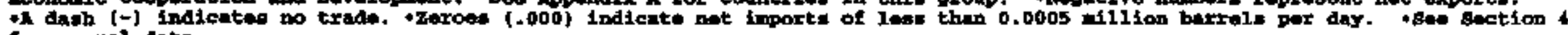
for annusl atte.

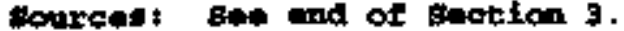


Table 3.2 wet oil Imoxts into Occo Countries, (3xd ouarter 1997) (fillion Berrels perr Day)

\begin{tabular}{|c|c|c|c|c|c|c|c|c|c|c|c|}
\hline & \multirow{2}{*}{ Totel } & \multicolumn{6}{|c|}{ Osen burope } & \multicolumn{4}{|c|}{ OxcD Outeide Europe } \\
\hline & & Total & $\begin{array}{l}\text { ontted } \\
\text { ringdol }\end{array}$ & Franos & aersungy & Italy & $\begin{array}{l}\text { Other } \\
\text { Europezy }\end{array}$ & $\begin{array}{l}\text { Onited } \\
\text { Otaten } /\end{array}$ & Cunnda & Japan & others \\
\hline 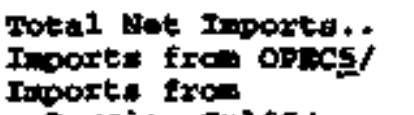 & $\begin{array}{l}21.975 \\
16.211\end{array}$ & $\begin{array}{l}7.621 \\
\text { 5.220 }\end{array}$ & $\begin{array}{r}-0.021 \\
.233\end{array}$ & $\begin{array}{r}1.715 \\
.965\end{array}$ & $\begin{array}{r}2.857 \\
.658\end{array}$ & $\begin{array}{l}1.708 \\
1.344\end{array}$ & $\begin{array}{l}2.162 \\
3.018\end{array}$ & $\begin{array}{l}6.990 \\
4.563\end{array}$ & $\begin{array}{r}-0.783 \\
.343\end{array}$ & $\begin{array}{l}5.646 \\
4.433\end{array}$ & $\begin{array}{r}0.501 \\
.652\end{array}$ \\
\hline 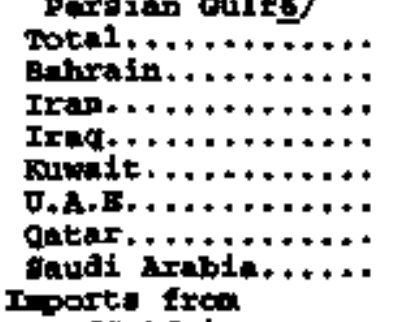 & $\begin{array}{l}9.728 \\
.019 \\
1.462 \\
.215 \\
1.119 \\
1.468 \\
.361 \\
5.064\end{array}$ & $\begin{array}{r}3.512 \\
.000 \\
1.022 \\
.159 \\
.304 \\
.009 \\
.000 \\
2.019\end{array}$ & $\begin{array}{l}.110 \\
.000 \\
.000 \\
.000 \\
.028 \\
.000 \\
.000 \\
.082\end{array}$ & $\begin{array}{l}.712 \\
.000 \\
.166 \\
.065 \\
.045 \\
.000 \\
.000 \\
.436\end{array}$ & $\begin{array}{l}.176 \\
.000 \\
.025 \\
.000 \\
.013 \\
.002 \\
.000 \\
.195\end{array}$ & $\begin{array}{l}.606 \\
.000 \\
.254 \\
.027 \\
.000 \\
.000 \\
.000 \\
.0324\end{array}$ & $\begin{array}{r}1.909 \\
.000 \\
.576 \\
.066 \\
.218 \\
.007 \\
.000 \\
1.041\end{array}$ & $\begin{array}{r}1.804 \\
-.003 \\
.000 \\
.030 \\
.294 \\
.000 \\
.000 \\
1.484\end{array}$ & $\begin{array}{l}.112 \\
- \\
.021 \\
.023 \\
.000 \\
.000 \\
- \\
.068\end{array}$ & $\begin{array}{r}3.979 \\
.016 \\
.419 \\
.000 \\
.520 \\
1.407 \\
.352 \\
1.266\end{array}$ & $\begin{array}{l}.320 \\
.006 \\
.000 \\
.003 \\
.002 \\
.073 \\
.009 \\
.227\end{array}$ \\
\hline 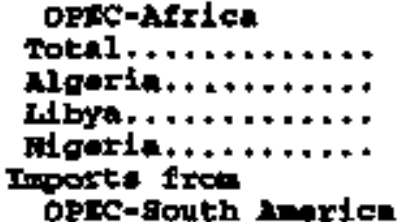 & $\begin{array}{l}3.762 \\
1.015 \\
1.257 \\
1.490\end{array}$ & $\begin{array}{r}2.455 \\
.650 \\
1.257 \\
.549\end{array}$ & $\begin{array}{l}.090 \\
.018 \\
.062 \\
.010\end{array}$ & $\begin{array}{l}.253 \\
.118 \\
.068 \\
.068\end{array}$ & $\begin{array}{l}.423 \\
.081 \\
.264 \\
.078\end{array}$ & $\begin{array}{l}.697 \\
.078 \\
.599 \\
.020\end{array}$ & $\begin{array}{l}.992 \\
.355 \\
.264 \\
.373\end{array}$ & $\begin{array}{r}1.012 \\
.270 \\
.000 \\
.741\end{array}$ & $\begin{array}{l}.100 \\
.067 \\
.000 \\
.032\end{array}$ & $\begin{array}{l}.066 \\
.027 \\
- \\
.039\end{array}$ & $\begin{array}{l}.129 \\
.000 \\
.000 \\
.129\end{array}$ \\
\hline 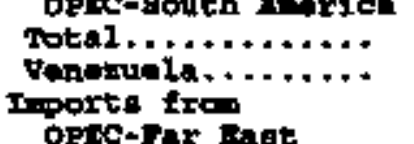 & $\begin{array}{l}2.156 \\
2.156\end{array}$ & $\begin{array}{r}.231 \\
.231\end{array}$ & $\begin{array}{l}.034 \\
.034\end{array}$ & $\begin{array}{r}.000 \\
.000\end{array}$ & $\begin{array}{l}.059 \\
.059\end{array}$ & $\begin{array}{l}.027 \\
.027\end{array}$ & $\begin{array}{l}.110 \\
.110\end{array}$ & $\begin{array}{l}1.688 \\
1.688\end{array}$ &. $\begin{array}{l}.131 \\
.131\end{array}$ & $\begin{array}{r}.000 \\
.000\end{array}$ & $\begin{array}{l}.106 \\
.106\end{array}$ \\
\hline Total $\ldots \ldots \ldots \ldots \ldots$ & .584 & $\begin{array}{l}.021 \\
.021\end{array}$ & $\begin{array}{l}.000 \\
.000\end{array}$ & $\begin{array}{l}.000 \\
.000\end{array}$ & $\begin{array}{l}.000 \\
.000\end{array}$ & .013 & .000 & $\begin{array}{l}.056 \\
.055\end{array}$ & $\begin{array}{l}.000 \\
.000\end{array}$ & $\begin{array}{l}.404 \\
.404\end{array}$ & $\begin{array}{l}.103 \\
.103\end{array}$ \\
\hline
\end{tabular}

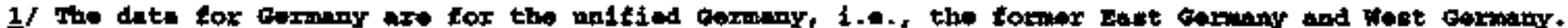

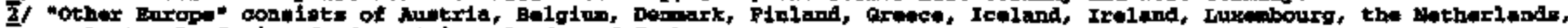

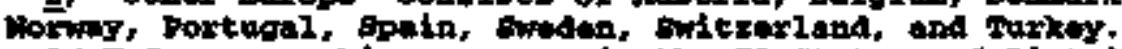

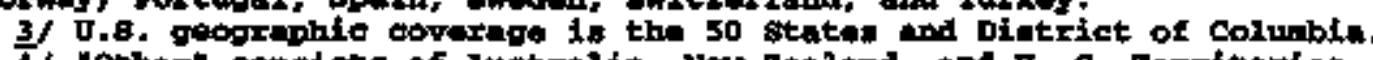

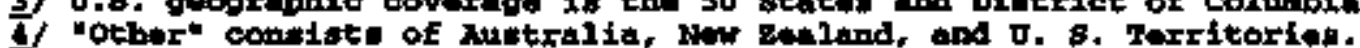

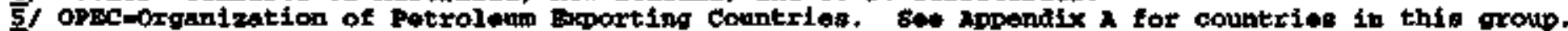

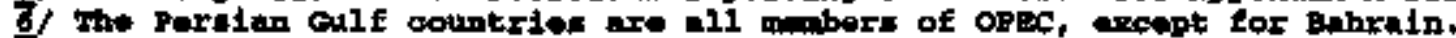

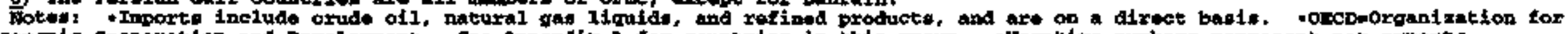

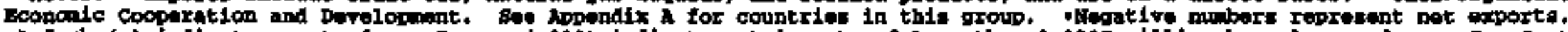

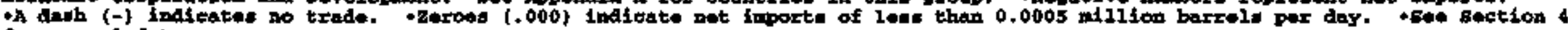

Lor andual data.

forrete; Bes and of teaction 3. 
Table 3.3 net 011 Inorts into oson conntries, (2nd quarter 1997) (Milision Barrels par Day)

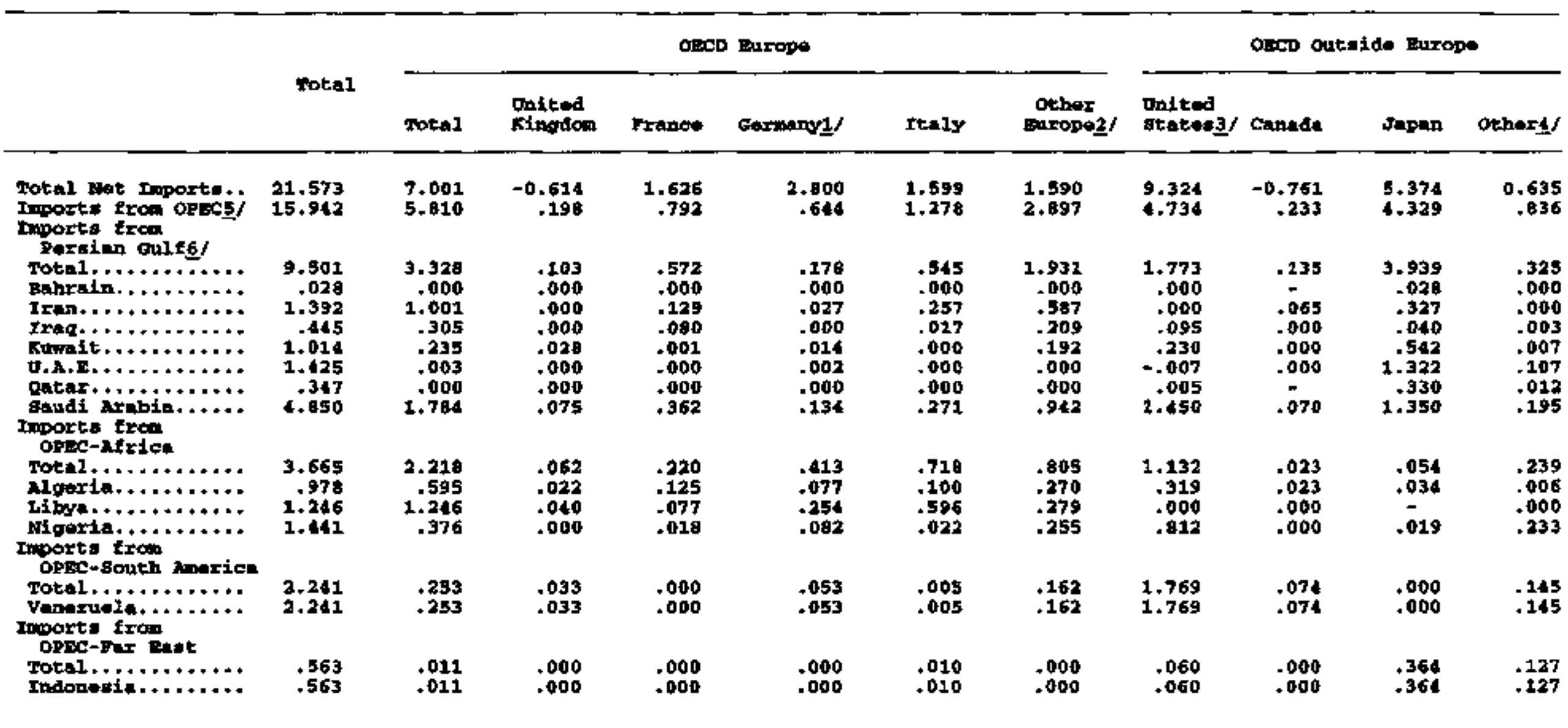

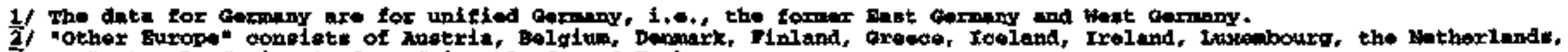
worway, Portugal, spain, Bwoden, thitzerland, and Turkey.

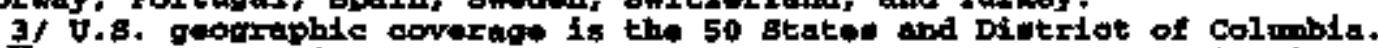

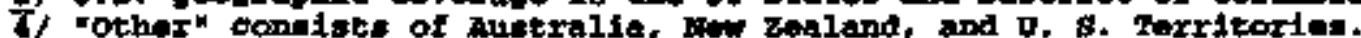

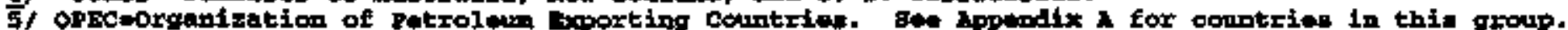

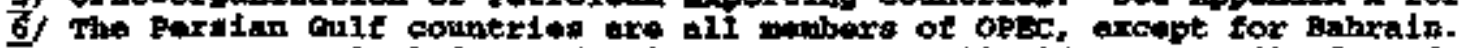

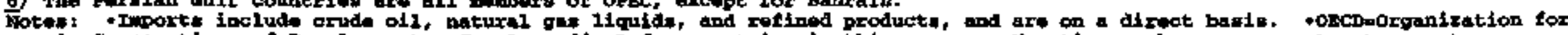

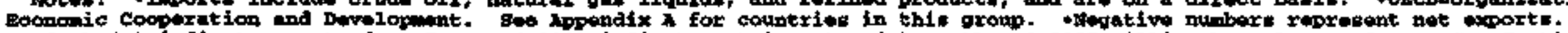

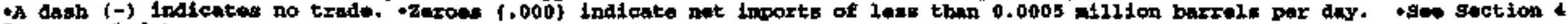
For annual data.

soureet see and of section 3 . 
Table 3.4 OSCD Total Wot Inporta (Wost Recent 12 wonths)

(Milizon Berrele pox Day)

1996

Hor Deo

Tan

Jas rab

nax

April

21.757

23.010
8.595

8.595

-.752
-.183

\subsection{7}

0.460
-.919

. .919
7.219

8,096

cranada ...........

Min maxcpent Imooxter.

Franct........... 1.861

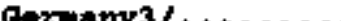

rtaly $\ldots \ldots \ldots \ldots \ldots$

spaln.

1.861
2.880

7.710

1. 919

1. 804

1.873

2. 905

1. 252

1.919
2.939

2.036

1. 239

1.992

2.665

1.655
1.953

1. 284

.747

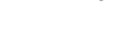

$-3.182$

$-3.100$

-3.100
-.995

$-3.157$

$-.952$

. .182

United ........

-3.482
-.712

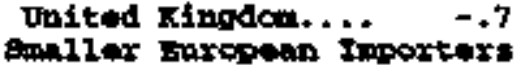

nustil.......... .218

belginn......... $\quad .590$

Demmerk.......... $\quad .038$

Irtand......... $\quad .106$

Finland......... .

Greot............

Igol and. $\ldots \ldots+\ldots \ldots$

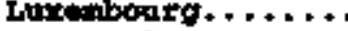

portugal ........

Biroden. . . . . . . . .

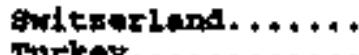

Torkey............

Tapan............

.438

.215

.665

143

.206

.727

$-.005$

.140

255

.355

.008

.041

.381

.498

. 381

.645

5.769

03항

.222

.449
.290

.630

6.013

.243

.411

.018

.039

.374

.236

.640

6.196

.796
.788

$+220$

$+510$

.062

.139

.147

.353

.010

.046

.217

.332

.270

.509

6.531

.605

21.350

21.350
0.780

.724

0.997
-.724
6.472

1.986

2.626

1.690

1.124

1.633

2.729
1.359

1. 359

1.253
.977

$-3.331$

.010

$-3.642$

$-.795$

.178

.661

.034

.093
.109

.335

.020

.043

.315

.440

.259

.521

6.298

.336

.317
.569

$-.062$

.165

.136
.332

.007

.040

.265

.423

250
.596

.596

$\begin{array}{r}637 \\ \hline\end{array}$

.610

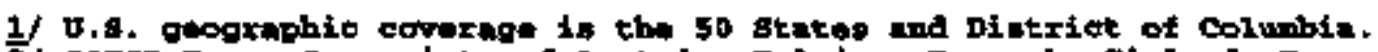

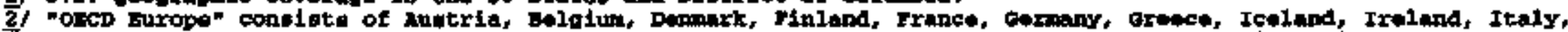

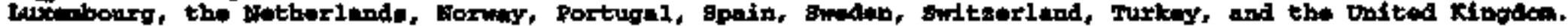

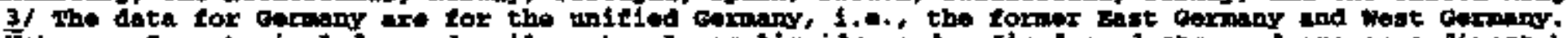

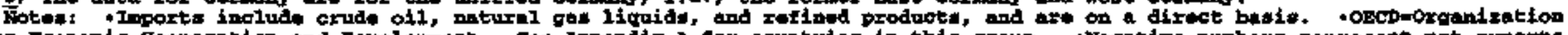

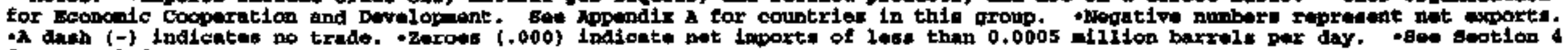
Cor ennugl date.

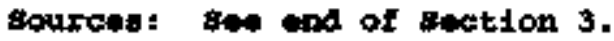

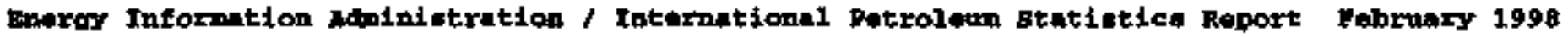


Table 3.5 OECD IPorts Eron OPIC (Nost Recent 12 wonthe)

(utillion Barrele por Day)

\begin{tabular}{|c|c|c|c|c|c|c|c|c|c|c|c|c|}
\hline & \multicolumn{2}{|c|}{1996} & \multicolumn{10}{|c|}{1997} \\
\hline & nov & Dee & Jan & Fab & $\operatorname{Hax}$ & $2 p+12$ & Way & Thos & $\operatorname{suly}$ & Aucoust & Sept & Oat \\
\hline 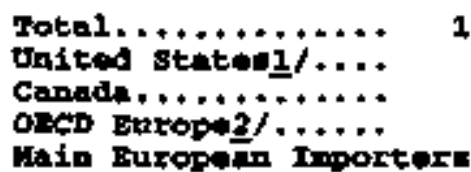 & $\begin{array}{r}15.926 \\
3.961 \\
. .274 \\
6.623\end{array}$ & $\begin{array}{r}15.893 \\
3.920 \\
.323 \\
6.213\end{array}$ & $\begin{array}{r}16.623 \\
4.066 \\
.313 \\
6.398\end{array}$ & $\begin{array}{r}16.233 \\
4.109 \\
.364 \\
3.994\end{array}$ & $\begin{array}{r}15.048 \\
4.251 \\
.274 \\
5.446\end{array}$ & $\begin{array}{r}16.289 \\
+.538 \\
.201 \\
5.811\end{array}$ & $\begin{array}{r}16.023 \\
4.886 \\
.242 \\
6.004\end{array}$ & $\begin{array}{r}15.511 \\
4.774 \\
5.255 \\
.608\end{array}$ & $\begin{array}{r}15.592 \\
4.273 \\
-293 \\
6.048\end{array}$ & $\begin{array}{r}16.255 \\
4.691 \\
. .457 \\
6.055\end{array}$ & $\begin{array}{r}16.806 \\
4.730 \\
.779 \\
6.568\end{array}$ & $\begin{array}{r}16.814 \\
4.800 \\
.393 \\
6.273\end{array}$ \\
\hline 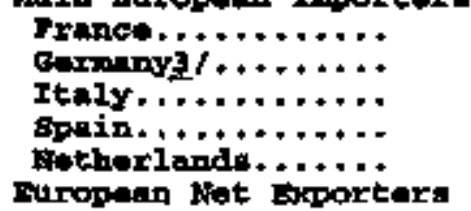 & $\begin{array}{r}1.003 \\
.653 \\
1.325 \\
.926 \\
.093\end{array}$ & $\begin{array}{r}.888 \\
.645 \\
1.372 \\
.864 \\
.911\end{array}$ & $\begin{array}{r}.820 \\
.605 \\
1.829 \\
.960 \\
.744\end{array}$ & $\begin{array}{r}1.095 \\
.618 \\
1.261 \\
.773 \\
.620\end{array}$ & $\begin{array}{r}.809 \\
.660 \\
1.270 \\
.630 \\
.598\end{array}$ & $\begin{array}{r}.802 \\
.669 \\
1.142 \\
.707 \\
.948\end{array}$ & $\begin{array}{r}.753 \\
.683 \\
1.484 \\
.703 \\
.767\end{array}$ & $\begin{array}{r}.024 \\
.591 \\
1.202 \\
.693 \\
.699\end{array}$ & $\begin{array}{r}1.020 \\
1.656 \\
1.379 \\
.764 \\
.759\end{array}$ & $\begin{array}{r}.797 \\
.682 \\
1.276 \\
.722 \\
.853\end{array}$ & $\begin{array}{r}1.094 \\
.636 \\
1.379 \\
.864 \\
.779\end{array}$ & $\begin{array}{r}1.131 \\
.649 \\
1.353 \\
.820 \\
.810\end{array}$ \\
\hline 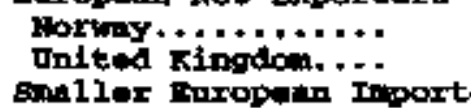 & $\begin{array}{r}.000 \\
.310 \\
\operatorname{tax}\end{array}$ & $\begin{array}{l}.000 \\
.188\end{array}$ & $\begin{array}{r}.009 \\
.179\end{array}$ & $\begin{array}{r}.007 \\
.215\end{array}$ & $\begin{array}{l}.000 \\
.176\end{array}$ & $\begin{array}{l}.000 \\
.176\end{array}$ & $\begin{array}{r}.000 \\
.199\end{array}$ & $\begin{array}{l}.000 \\
.219\end{array}$ & $\begin{array}{l}.000 \\
.271\end{array}$ & $\begin{array}{l}.000 \\
.190\end{array}$ & $\begin{array}{r}.000 \\
.239\end{array}$ & $\begin{array}{r}.007 \\
.170\end{array}$ \\
\hline 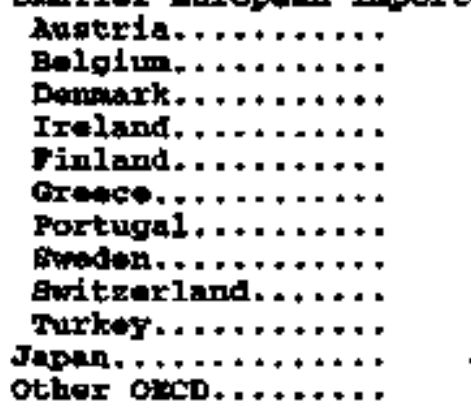 & $\begin{array}{l}.000 \\
.346 \\
.032 \\
.000 \\
.000 \\
.302 \\
.161 \\
.070 \\
.093 \\
.509 \\
4.502 \\
.577\end{array}$ & $\begin{array}{l}.000 \\
.238 \\
.044 \\
.000 \\
.000 \\
.297 \\
.145 \\
.105 \\
.079 \\
.436 \\
4.620 \\
.817\end{array}$ & $\begin{array}{l}.000 \\
.290 \\
.025 \\
.000 \\
.000 \\
.362 \\
.197 \\
.145 \\
.103 \\
.522 \\
5.026 \\
.020\end{array}$ & $\begin{array}{l}.000 \\
.223 \\
.052 \\
.000 \\
.000 \\
.293 \\
.168 \\
.104 \\
.109 \\
.095 \\
5.137 \\
.629\end{array}$ & $\begin{array}{l}.000 \\
.157 \\
.000 \\
.000 \\
.000 \\
.275 \\
.220 \\
.150 \\
.097 \\
.104 \\
5.236 \\
.642\end{array}$ & $\begin{array}{l}.000 \\
.290 \\
.025 \\
.000 \\
.000 \\
.284 \\
.142 \\
.227 \\
.063 \\
.436 \\
.839 \\
.900\end{array}$ & $\begin{array}{l}.000 \\
.315 \\
.049 \\
.000 \\
.010 \\
.286 \\
.180 \\
.099 \\
.087 \\
.377 \\
.175 \\
.717\end{array}$ & $\begin{array}{r}.000 \\
.309 \\
.033 \\
.000 \\
.000 \\
.294 \\
.179 \\
.092 \\
.093 \\
.390 \\
3.977 \\
.096\end{array}$ & $\begin{array}{l}.000 \\
.244 \\
.006 \\
.000 \\
.000 \\
.300 \\
.177 \\
.067 \\
.094 \\
.311 \\
6.357 \\
.621\end{array}$ & $\begin{array}{r}.000 \\
.326 \\
.027 \\
.000 \\
.000 \\
.333 \\
.156 \\
.089 \\
.101 \\
.502 \\
. .416 \\
.636\end{array}$ & $\begin{array}{r}.000 \\
.332 \\
.067 \\
.000 \\
.008 \\
.299 \\
.206 \\
.025 \\
.107 \\
.522 \\
4.527 \\
.702\end{array}$ & $\begin{array}{r}.000 \\
.294 \\
.024 \\
.000 \\
.011 \\
.280 \\
.080 \\
.086 \\
.111 \\
.447 \\
4.732 \\
.616\end{array}$ \\
\hline
\end{tabular}

1/ ס.8. geographis coverage is the 50 gtates and Dintriet of Colunibil.

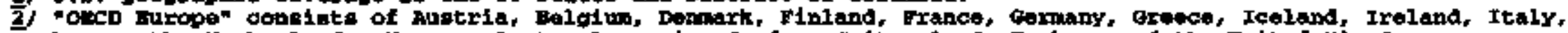

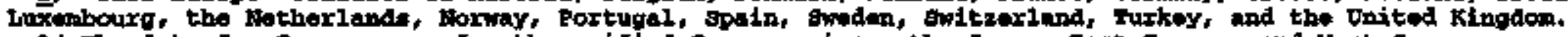

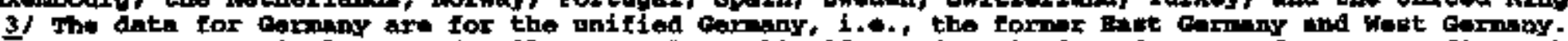

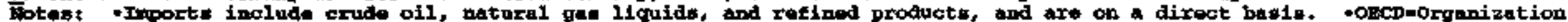

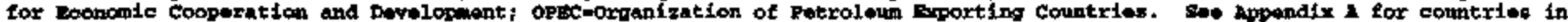

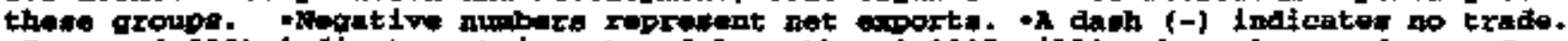

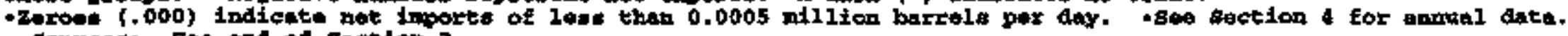
Jourcesis bee end of section 3 . 
Table 3.6 obed Iportu frow Perwian Gulf (wost Recent 12 wonthe) (willice Barrele perr Day)

\begin{tabular}{|c|c|c|c|c|c|c|c|c|c|c|c|c|}
\hline & \multicolumn{2}{|c|}{1996} & \multicolumn{10}{|c|}{1997} \\
\hline & Nort & Dec & $\operatorname{Tan}$ & $F=b$ & Mner & Apri1 & uny & rune & July & Auguet & sopt & $\alpha c t$ \\
\hline 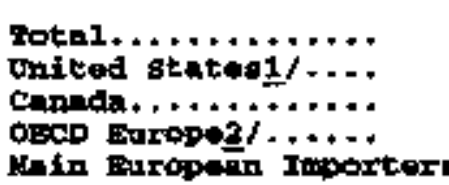 & $\begin{array}{r}9.652 \\
1.519 \\
.090 \\
3.557 \\
\approx^{5}\end{array}$ & $\begin{array}{l}9.622 \\
1.673 \\
.128 \\
3.133\end{array}$ & $\begin{array}{r}9.986 \\
1.548 \\
+130 \\
3.322\end{array}$ & $\begin{array}{r}9.914 \\
1.539 \\
.146 \\
3.159\end{array}$ & $\begin{array}{l}9.569 \\
1.625 \\
.067 \\
2.057\end{array}$ & $\begin{array}{r}10.003 \\
1.059 \\
.133 \\
3.223\end{array}$ & $\begin{array}{l}9.565 \\
1.701 \\
.137 \\
3.504\end{array}$ & $\begin{array}{r}0.931 \\
1.763 \\
.136 \\
3.169\end{array}$ & $\begin{array}{r}9.292 \\
1.712 \\
.066 \\
3.239\end{array}$ & $\begin{array}{r}9.782 \\
1.836 \\
.198 \\
3.510\end{array}$ & $\begin{array}{r}10.122 \\
1.867 \\
.071 \\
3.797\end{array}$ & $\begin{array}{r}10.501 \\
1.876 \\
.176 \\
3.718\end{array}$ \\
\hline 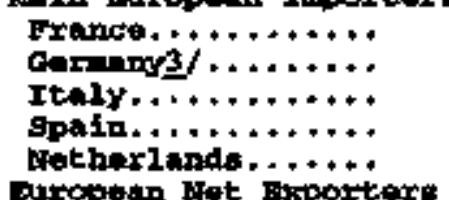 & $\begin{array}{l}.642 \\
.167 \\
.543 \\
.327 \\
.644\end{array}$ & $\begin{array}{l}.569 \\
.106 \\
.532 \\
.288 \\
.661\end{array}$ & $\begin{array}{l}.535 \\
.088 \\
.550 \\
.340 \\
.568\end{array}$ & $\begin{array}{l}.753 \\
.094 \\
.599 \\
.301 \\
.499\end{array}$ & $\begin{array}{l}.404 \\
.131 \\
.507 \\
.355 \\
.456\end{array}$ & $\begin{array}{l}.599 \\
.189 \\
.458 \\
.388 \\
.569\end{array}$ & $\begin{array}{l}.555 \\
.153 \\
.646 \\
.413 \\
.642\end{array}$ & $\begin{array}{l}.563 \\
.192 \\
.526 \\
.287 \\
.515\end{array}$ & $\begin{array}{l}.649 \\
.176 \\
.618 \\
.318 \\
.554\end{array}$ & $\begin{array}{l}.644 \\
.187 \\
.371 \\
.358 \\
.583\end{array}$ & $\begin{array}{r}.057 \\
.164 \\
.629 \\
-425 \\
.495\end{array}$ & $\begin{array}{l}.642 \\
.205 \\
.645 \\
.011 \\
.670\end{array}$ \\
\hline 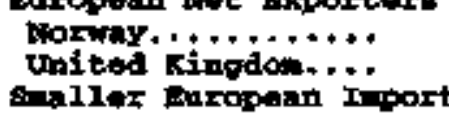 & $\begin{array}{l}.000 \\
+122 \\
\operatorname{tar} \%\end{array}$ & $\begin{array}{l}.000 \\
.067\end{array}$ & $\begin{array}{l}.000 \\
.066\end{array}$ & $\begin{array}{l}.000 \\
.059\end{array}$ & $\begin{array}{l}.000 \\
.084\end{array}$ & $\begin{array}{r}.000 \\
.078\end{array}$ & $\begin{array}{l}.000 \\
.107\end{array}$ & .000 & .000 & $\begin{array}{l}.000 \\
.091\end{array}$ & .000 & $\begin{array}{l}.000 \\
.081\end{array}$ \\
\hline 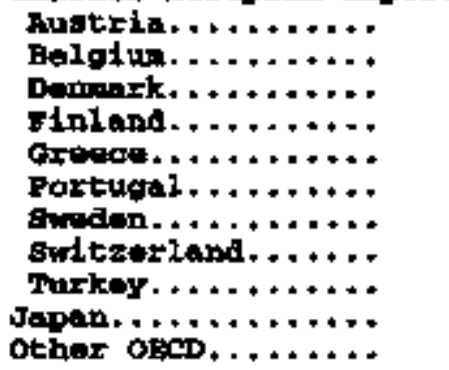 & $\begin{array}{r}.000 \\
.331 \\
.000 \\
.000 \\
.270 \\
.008 \\
.070 \\
.005 \\
.348 \\
+223 \\
.264\end{array}$ & $\begin{array}{l}.000 \\
.219 \\
-000 \\
.000 \\
.245 \\
.100 \\
.061 \\
.000 \\
.286 \\
4.219 \\
.869\end{array}$ & $\begin{array}{r}.000 \\
.250 \\
.000 \\
.000 \\
.317 \\
.091 \\
.132 \\
.000 \\
.385 \\
+.616 \\
.369\end{array}$ & $\begin{array}{l}.000 \\
.191 \\
.000 \\
.000 \\
.250 \\
.057 \\
.070 \\
.005 \\
.287 \\
4.750 \\
.328\end{array}$ & $\begin{array}{l}.000 \\
.119 \\
.000 \\
.000 \\
.232 \\
.161 \\
.133 \\
.005 \\
.267 \\
.705 \\
.315\end{array}$ & $\begin{array}{l}.000 \\
.233 \\
.000 \\
.000 \\
.251 \\
.052 \\
.070 \\
.010 \\
.327 \\
4.199 \\
.291\end{array}$ & $\begin{array}{l}.000 \\
.285 \\
.000 \\
.000 \\
.254 \\
.161 \\
.061 \\
.014 \\
.289 \\
3.816 \\
.327\end{array}$ & $\begin{array}{l}.000 \\
+250 \\
.000 \\
.000 \\
.243 \\
.138 \\
.067 \\
.014 \\
.249 \\
.505 \\
.357\end{array}$ & $\begin{array}{l}.000 \\
.238 \\
.000 \\
.000 \\
.266 \\
.062 \\
.067 \\
.009 \\
.178 \\
3.884 \\
.392\end{array}$ & $\begin{array}{r}.000 \\
.292 \\
.000 \\
.000 \\
.298 \\
.087 \\
.065 \\
.009 \\
.333 \\
3.955 \\
.282\end{array}$ & $\begin{array}{l}-000 \\
+331 \\
.023 \\
.000 \\
.262 \\
.119 \\
.000 \\
.009 \\
.370 \\
+103 \\
.264\end{array}$ & $\begin{array}{r}.000 \\
.277 \\
.000 \\
.000 \\
.239 \\
.023 \\
.066 \\
.014 \\
.244 \\
+.497 \\
.234\end{array}$ \\
\hline
\end{tabular}

1/ 0.a. geograpbic coverige is the 50 gtates and District of columis.

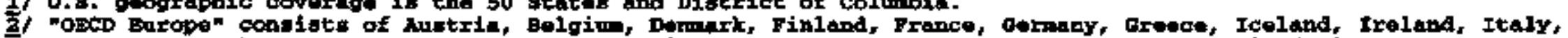

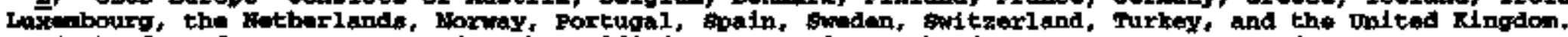

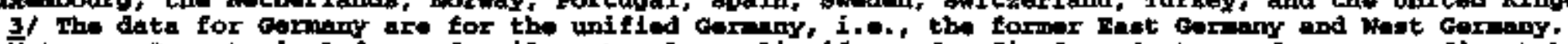

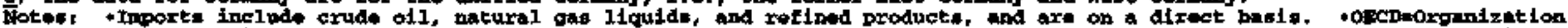

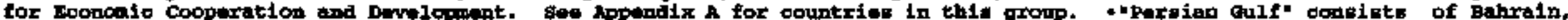

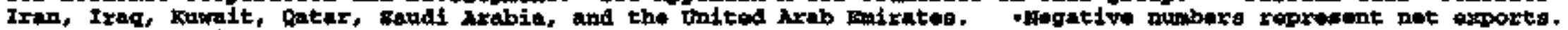

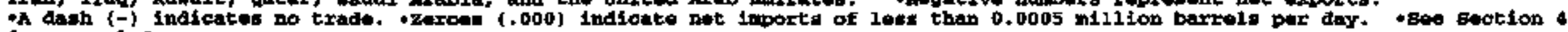
for anmul dita.

gonmeset get end of stotion 3 . 


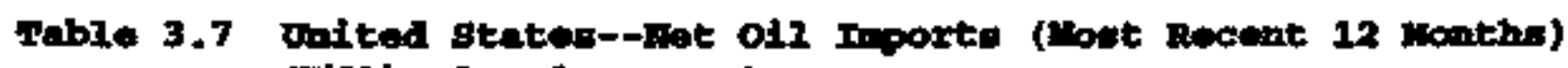
(allilon sarrole per Day)

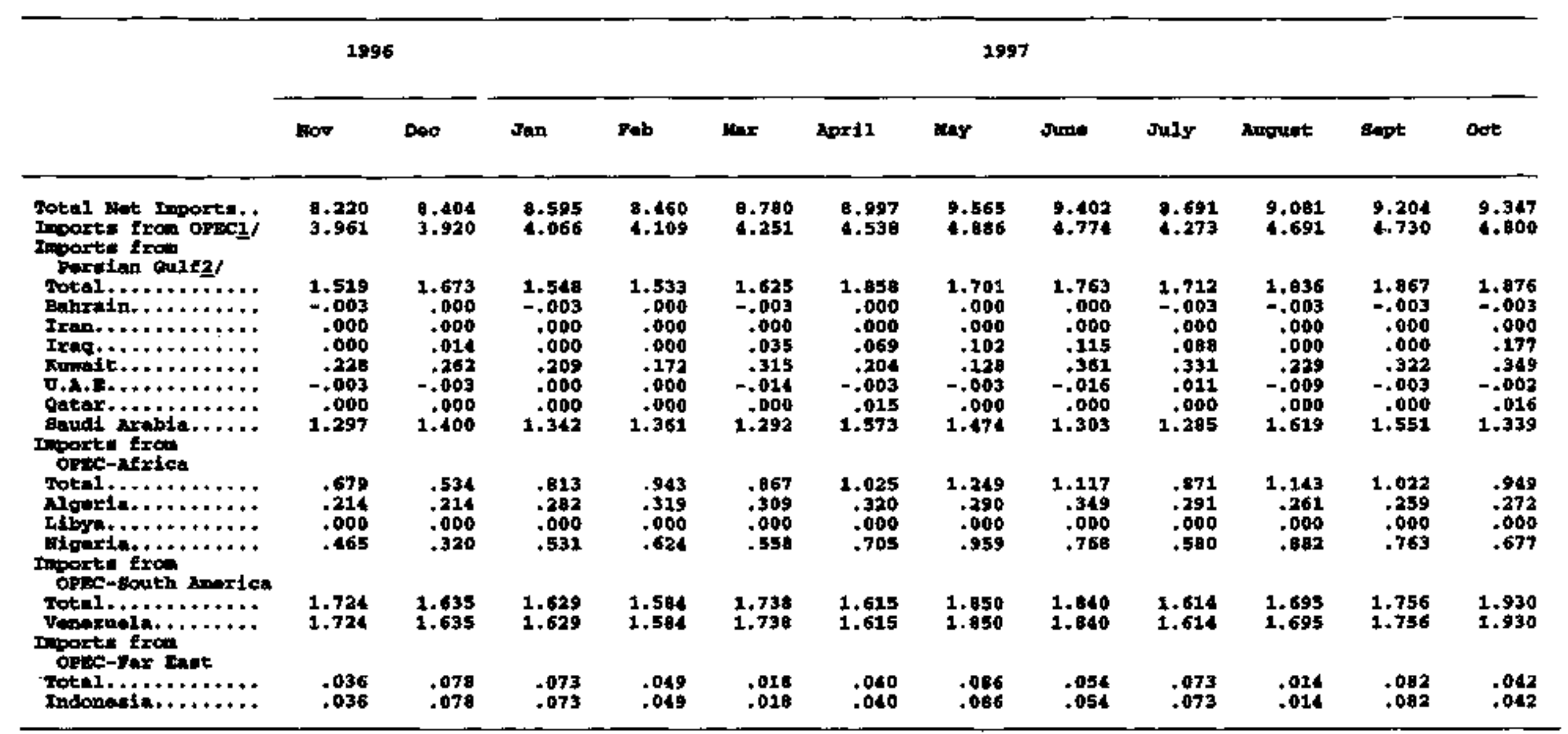

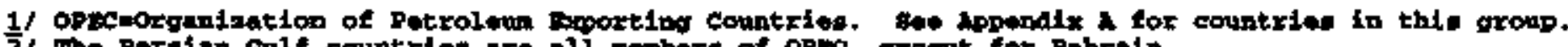

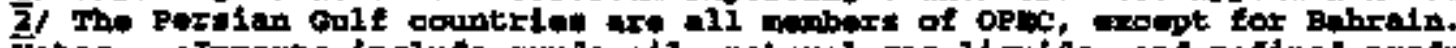

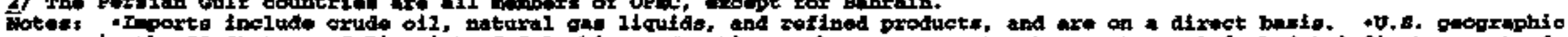

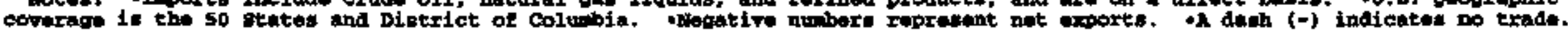

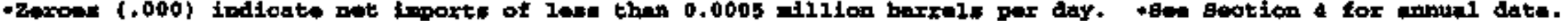

Bourcest see and of section 3 . 


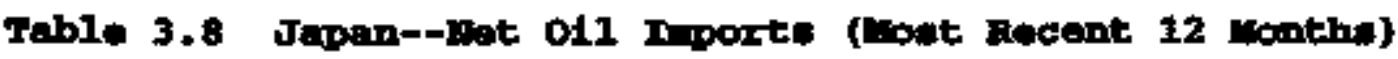

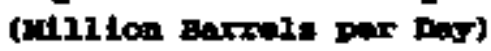

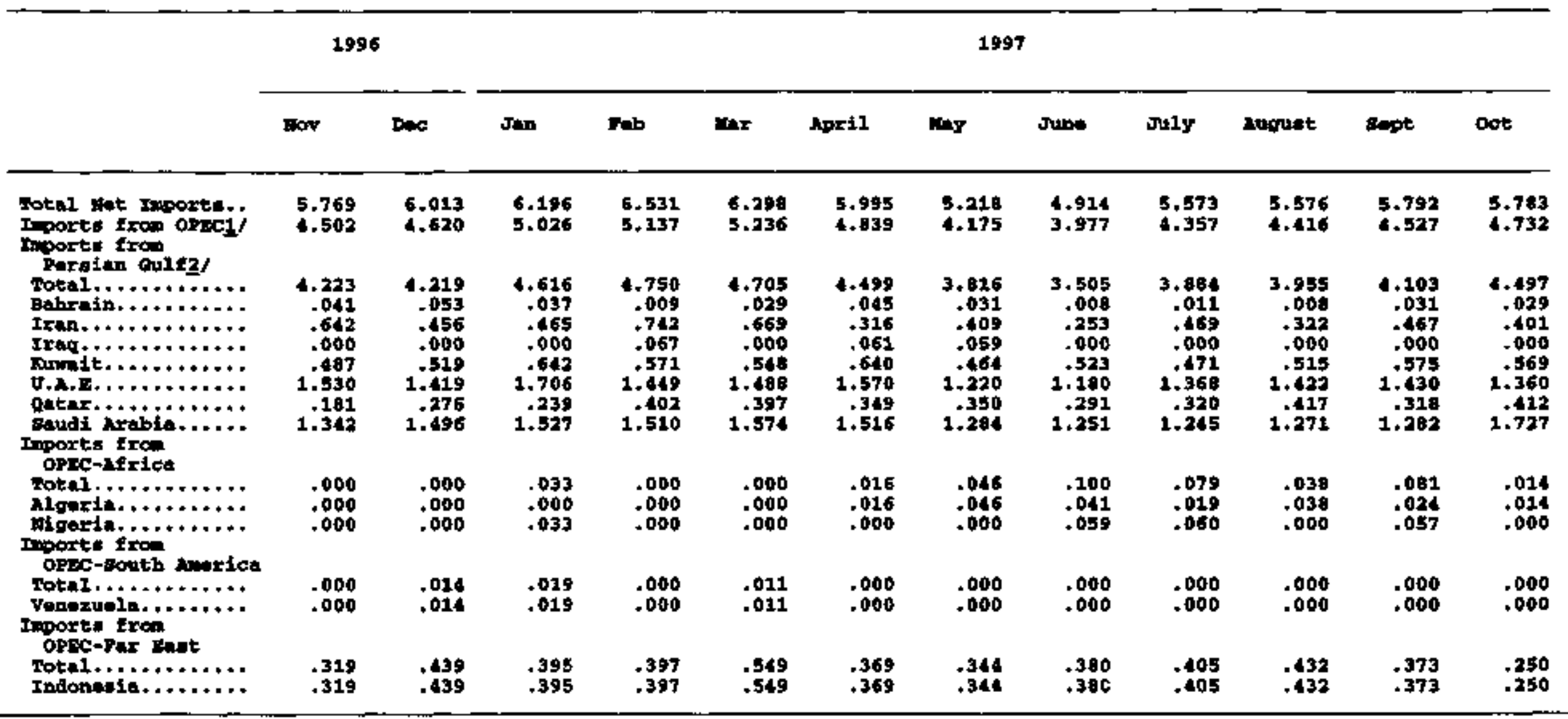

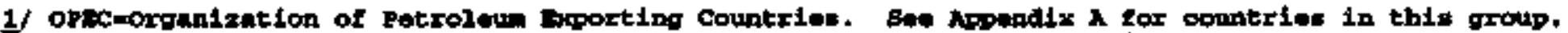

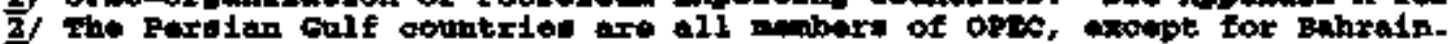

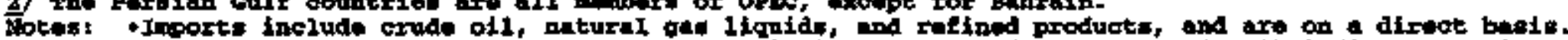

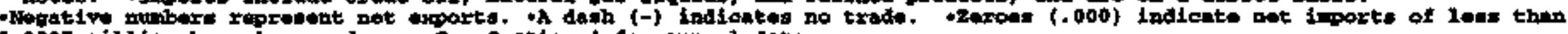

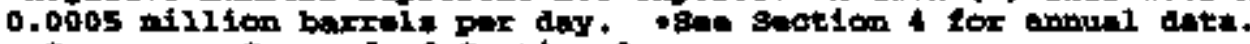

hources: keo ond of bection 3 . 
Table 3.9 OacD buropo--10t O11 Imports (Wost Recent 12 Fonths)

(MIllion Barrole par Dar)

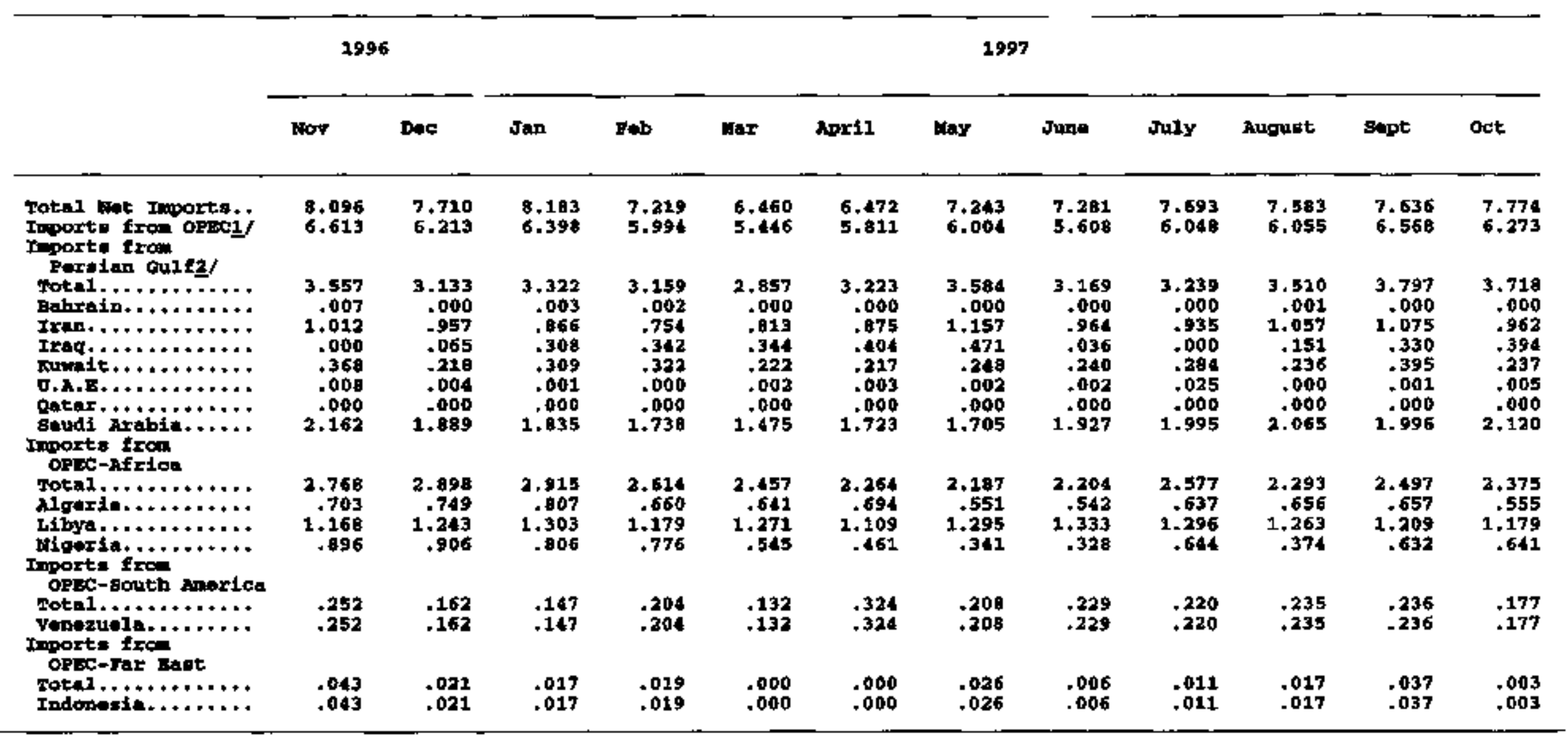

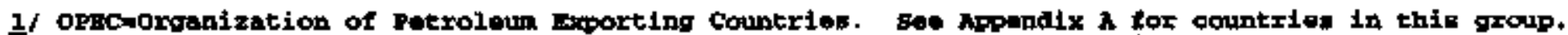

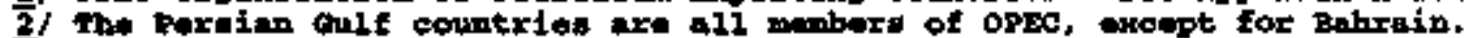

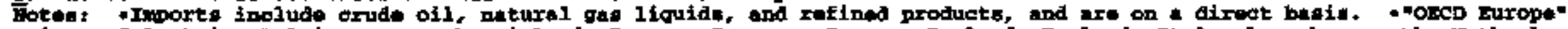

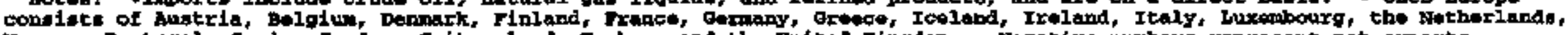

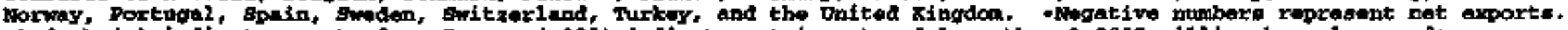

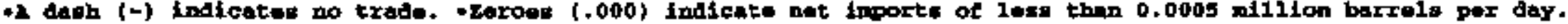

- Sow frection 4 for anmul data.

Sotrese: tee and of section 3 . 
Table 3.10 France--10t oil Iroorts (Woat Recont 12 wonth") (atilicon berrols por Day)

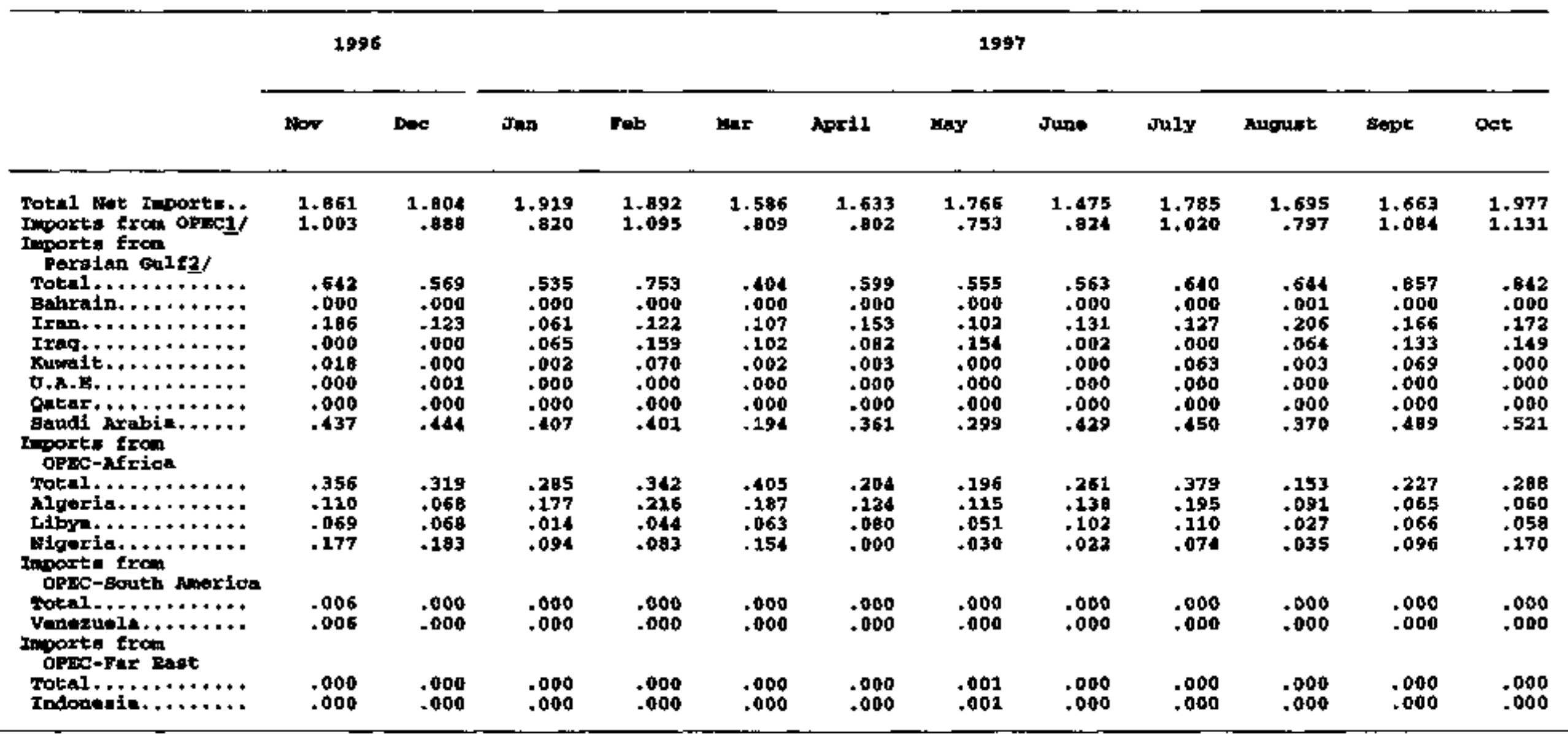

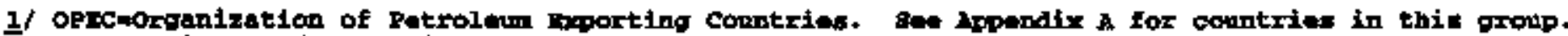

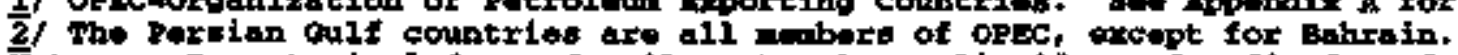

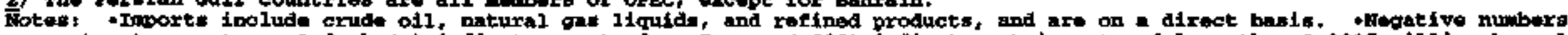

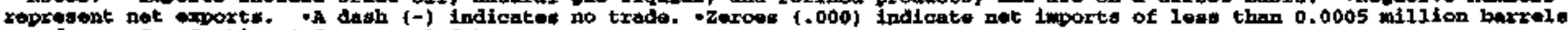
ber day. ¿840 Bection 4 Eor annual deta.

souxses: tan and of Beotion 3 . 


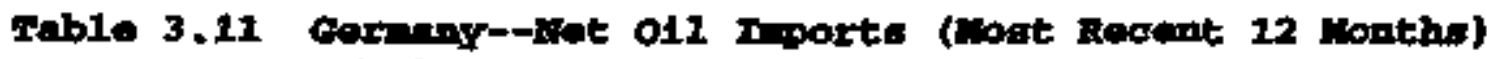
(arlilan Barcele per Day)

\begin{tabular}{|c|c|c|c|c|c|c|c|c|c|c|c|c|}
\hline & \multicolumn{2}{|c|}{1996} & \multicolumn{10}{|c|}{1997} \\
\hline & Mop & Dect & $7 m$ & $P=b$ & Lar & $\mathbf{A b r | 1 1}$ & May & The & ruly & Rapust & sept & oot \\
\hline 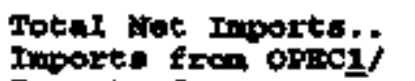 & $\begin{array}{r}2.880 \\
.653\end{array}$ & $\begin{array}{r}2.805 \\
.645\end{array}$ & $\begin{array}{r}2.939 \\
.605\end{array}$ & $\begin{array}{r}2.665 \\
.618\end{array}$ & $\begin{array}{r}2.626 \\
.660\end{array}$ & $\begin{array}{r}2.729 \\
.668\end{array}$ & $\begin{array}{r}2.963 \\
.683\end{array}$ & $\begin{array}{r}2.703 \\
.591\end{array}$ & $\begin{array}{r}3.129 \\
.656\end{array}$ & $\begin{array}{r}2.674 \\
.682\end{array}$ & $\begin{array}{r}2.766 \\
.636\end{array}$ & $\begin{array}{r}2.711 \\
.649\end{array}$ \\
\hline 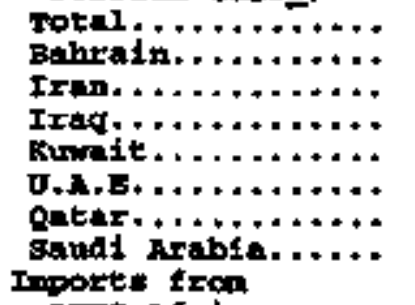 & $\begin{array}{l}.167 \\
.000 \\
.018 \\
.000 \\
.028 \\
.000 \\
.000 \\
.122\end{array}$ & $\begin{array}{l}.106 \\
.000 \\
.000 \\
.000 \\
.011 \\
.000 \\
.000 \\
.094\end{array}$ & $\begin{array}{l}.098 \\
.000 \\
.012 \\
.000 \\
.009 \\
.000 \\
.000 \\
.060\end{array}$ & $\begin{array}{l}.094 \\
.000 \\
.011 \\
.000 \\
.009 \\
.000 \\
.040 \\
.074\end{array}$ & $\begin{array}{l}.131 \\
.000 \\
.028 \\
.000 \\
.010 \\
.000 \\
.000 \\
.094\end{array}$ & $\begin{array}{l}.1 .89 \\
.000 \\
.029 \\
.000 \\
.020 \\
.002 \\
.000 \\
.137\end{array}$ & $\begin{array}{l}.153 \\
.000 \\
.016 \\
.000 \\
.009 \\
.002 \\
.000 \\
.125\end{array}$ & $\begin{array}{l}.192 \\
.000 \\
.037 \\
.000 \\
.013 \\
.002 \\
.000 \\
.140\end{array}$ & $\begin{array}{r}.176 \\
.000 \\
.022 \\
.000 \\
.014 \\
.005 \\
.000 \\
.135\end{array}$ & $\begin{array}{l}.107 \\
.000 \\
.037 \\
.000 \\
.009 \\
.000 \\
.000 \\
.141\end{array}$ & $\begin{array}{l}.164 \\
.000 \\
.017 \\
.000 \\
.016 \\
.000 \\
.000 \\
.130\end{array}$ & $\begin{array}{l}.205 \\
.004 \\
.041 \\
.000 \\
.012 \\
.000 \\
.000 \\
.152\end{array}$ \\
\hline 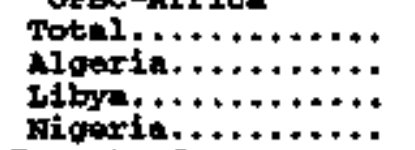 & $\begin{array}{l}.029 \\
.084 \\
.232 \\
.112\end{array}$ & $\begin{array}{r}.511 \\
.139 \\
.250 \\
.121\end{array}$ & $\begin{array}{l}.503 \\
.108 \\
.276 \\
.118\end{array}$ & $\begin{array}{l}.505 \\
.100 \\
.226 \\
.189\end{array}$ & $\begin{array}{l}.185 \\
.108 \\
.256 \\
.121\end{array}$ & $\begin{array}{l}.416 \\
.108 \\
.182 \\
.126\end{array}$ & $\begin{array}{l}.470 \\
.054 \\
.318 \\
.097\end{array}$ & $\begin{array}{l}.352 \\
.070 \\
.261 \\
.021\end{array}$ & $\begin{array}{l}.378 \\
.061 \\
.252 \\
.066\end{array}$ & $\begin{array}{l}.459 \\
.092 \\
.273 \\
.095\end{array}$ & $\begin{array}{l}.431 \\
.091 \\
.267 \\
.073\end{array}$ & $\begin{array}{l}.401 \\
.106 \\
.215 \\
.080\end{array}$ \\
\hline $\begin{array}{l}\text { Imports from } \\
\text { op:e-south norion }\end{array}$ & & & & & & & & & & & & \\
\hline 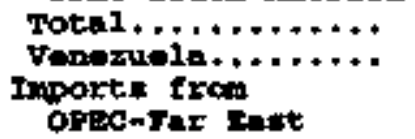 & $\begin{array}{l}.05 \theta \\
.05 \theta\end{array}$ & $\begin{array}{r}.029 \\
.029\end{array}$ & $\begin{array}{l}.014 \\
.014\end{array}$ & $\begin{array}{l}.018 \\
.018\end{array}$ & $\begin{array}{r}.043 \\
.043\end{array}$ & $\begin{array}{l}.064 \\
.064\end{array}$ & $\begin{array}{l}.059 \\
.059\end{array}$ & $\begin{array}{r}.037 \\
.037\end{array}$ & $\begin{array}{r}.101 \\
.101\end{array}$ & $\begin{array}{r}.036 \\
.036\end{array}$ & $\begin{array}{l}.041 \\
.041\end{array}$ & $\begin{array}{r}.043 \\
.043\end{array}$ \\
\hline 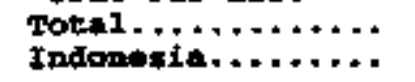 & $\begin{array}{l}.000 \\
.000\end{array}$ & .000 & $\begin{array}{l}.000 \\
.000\end{array}$ & $\begin{array}{l}.000 \\
.000\end{array}$ & $\begin{array}{l}.000 \\
.000\end{array}$ & $\begin{array}{l}.000 \\
.000\end{array}$ & $\begin{array}{l}.001 \\
.001\end{array}$ & $\begin{array}{l}.000 \\
.000\end{array}$ & $\begin{array}{l}.001 \\
.001\end{array}$ & $\begin{array}{l}.000 \\
.000\end{array}$ & $\begin{array}{l}.000 \\
.000\end{array}$ & $\begin{array}{l}.000 \\
.000\end{array}$ \\
\hline
\end{tabular}

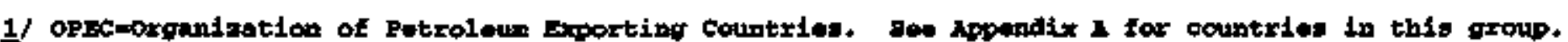

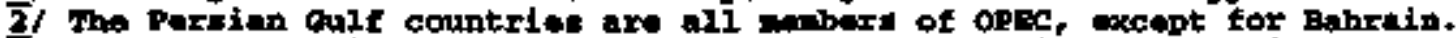

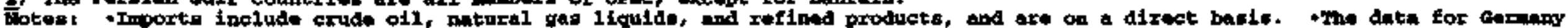

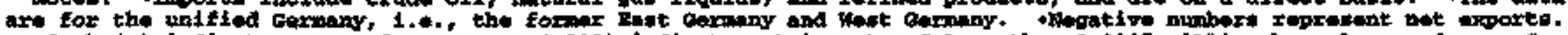

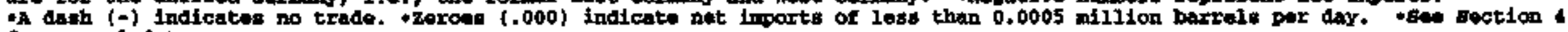
for annual Ante.

Soscens see ond of section 3. 
Table 3.12 Italy--170t oil Iporte (Wost Recent 12 woaths)

(IIllion Barrule gar Day)

\begin{tabular}{|c|c|c|c|c|c|c|c|c|c|c|c|c|}
\hline & \multicolumn{2}{|c|}{1996} & \multicolumn{10}{|c|}{1997} \\
\hline & wor & bes & Jan & Fab & Her & Aprti & Woy & June & $\operatorname{paly}$ & awgut & Dopt & oct \\
\hline $\begin{array}{l}\text { notal bot Inoorts; } \\
\text { Iipoxt fron oprci; }\end{array}$ & $\begin{array}{l}1.973 \\
1.325\end{array}$ & $\begin{array}{l}1.911 \\
1.372\end{array}$ & $\begin{array}{l}2.036 \\
1.429\end{array}$ & $\begin{array}{l}1.953 \\
1.281\end{array}$ & $\begin{array}{l}1.690 \\
1.270\end{array}$ & $\begin{array}{l}1.359 \\
1.142\end{array}$ & $\begin{array}{l}1.794 \\
1.484\end{array}$ & $\begin{array}{l}1.638 \\
1.202\end{array}$ & $\begin{array}{l}1.666 \\
1.379\end{array}$ & $\begin{array}{l}1.662 \\
1.276\end{array}$ & $\begin{array}{l}1.800 \\
1.379\end{array}$ & $\begin{array}{l}1.692 \\
1.353\end{array}$ \\
\hline 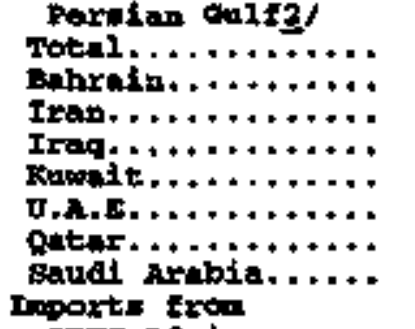 & $\begin{array}{l}.543 \\
.007 \\
.209 \\
.000 \\
.000 \\
.000 \\
.000 \\
.327\end{array}$ & $\begin{array}{l}.532 \\
.060 \\
.238 \\
.000 \\
.000 \\
.000 \\
.000 \\
.294\end{array}$ & $\begin{array}{l}.550 \\
.003 \\
.241 \\
.048 \\
.000 \\
.000 \\
.000 \\
.258\end{array}$ & $\begin{array}{l}.589 \\
.002 \\
.349 \\
.000 \\
.000 \\
.000 \\
.000 \\
.0038\end{array}$ & $\begin{array}{r}.507 \\
.000 \\
.250 \\
.032 \\
.000 \\
.002 \\
.000 \\
.223\end{array}$ & $\begin{array}{r}.158 \\
.000 \\
.147 \\
.000 \\
.000 \\
.000 \\
.000 \\
.311\end{array}$ & $\begin{array}{l}.646 \\
.000 \\
.365 \\
.049 \\
.000 \\
.000 \\
.000 \\
.232\end{array}$ & $\begin{array}{r}\mathbf{5 2 6} \\
.000 \\
+256 \\
.000 \\
.000 \\
+000 \\
+000 \\
+270\end{array}$ & $\begin{array}{l}.018 \\
.000 \\
.292 \\
.000 \\
.000 \\
.000 \\
.000 \\
.386\end{array}$ & $\begin{array}{r}.571 \\
.000 \\
.259 \\
.021 \\
.000 \\
.000 \\
.000 \\
.291\end{array}$ & $\begin{array}{r}.629 \\
.000 \\
.272 \\
.062 \\
.000 \\
.000 \\
.000 \\
.295\end{array}$ & $\begin{array}{l}.649 \\
.000 \\
.261 \\
.095 \\
.001 \\
.000 \\
.000 \\
.208\end{array}$ \\
\hline 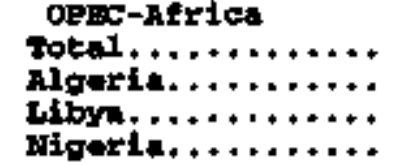 & $\begin{array}{l}.742 \\
.094 \\
.576 \\
.071\end{array}$ & $\begin{array}{l}.820 \\
.122 \\
.637 \\
.061\end{array}$ & $\begin{array}{l}.848 \\
.110 \\
.632 \\
.105\end{array}$ & $\begin{array}{l}.672 \\
.084 \\
.524 \\
.065\end{array}$ & $\begin{array}{l}.756 \\
.073 \\
.617 \\
.066\end{array}$ & $\begin{array}{l}.669 \\
.141 \\
.492 \\
.036\end{array}$ & $\begin{array}{l}.814 \\
.101 \\
.684 \\
.029\end{array}$ & $\begin{array}{l}.669 \\
-059 \\
.610 \\
.000\end{array}$ & $\begin{array}{l}.699 \\
.059 \\
.612 \\
.030\end{array}$ & $\begin{array}{l}.670 \\
.073 \\
.597 \\
.000\end{array}$ & $\begin{array}{l}.723 \\
.104 \\
.589 \\
.031\end{array}$ & $\begin{array}{l}.705 \\
.056 \\
.600 \\
.048\end{array}$ \\
\hline $\begin{array}{l}\text { Inports fron } \\
\text { OPEC-8puth norice }\end{array}$ & & & & & & & & & & & & \\
\hline 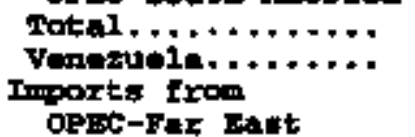 & $\begin{array}{l}.039 \\
.029\end{array}$ & $\begin{array}{l}.000 \\
.000\end{array}$ & $\begin{array}{l}.017 \\
.027\end{array}$ & $\begin{array}{l}.004 \\
.004\end{array}$ & $\begin{array}{r}.007 \\
.007\end{array}$ & .016 & $\begin{array}{l}.000 \\
.000\end{array}$ & $\begin{array}{l}.000 \\
.000\end{array}$ & .051 & $\begin{array}{l}.018 \\
.016\end{array}$ & $\begin{array}{l}.013 \\
.013\end{array}$ & .000 \\
\hline Toten1,........... & $\begin{array}{l}.018 \\
.018\end{array}$ & $\begin{array}{l}.021 \\
.021\end{array}$ & $\begin{array}{l}.017 \\
.027\end{array}$ & $\begin{array}{l}.018 \\
.018\end{array}$ & $\begin{array}{l}.000 \\
.000\end{array}$ & $\begin{array}{l}.000 \\
.000\end{array}$ & .024 & $\begin{array}{l}.006 \\
.006\end{array}$ & $\begin{array}{l}.010 \\
.010\end{array}$ & $\begin{array}{l}.017 \\
.017\end{array}$ & $\begin{array}{l}.013 \\
.013\end{array}$ & $\begin{array}{l}.003 \\
.003\end{array}$ \\
\hline
\end{tabular}

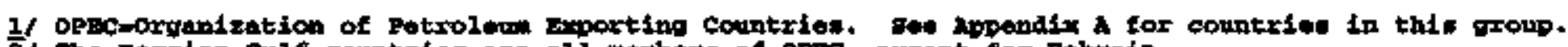

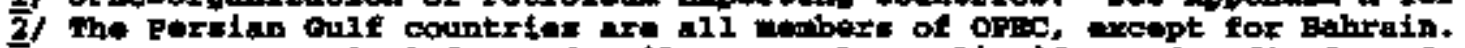

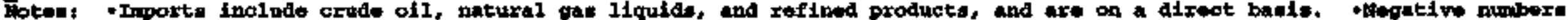

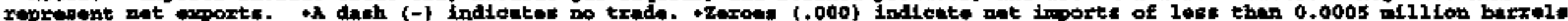
pax day. Boo geotion 4 for annum data.

sourets ; soo and of pection 3 . 


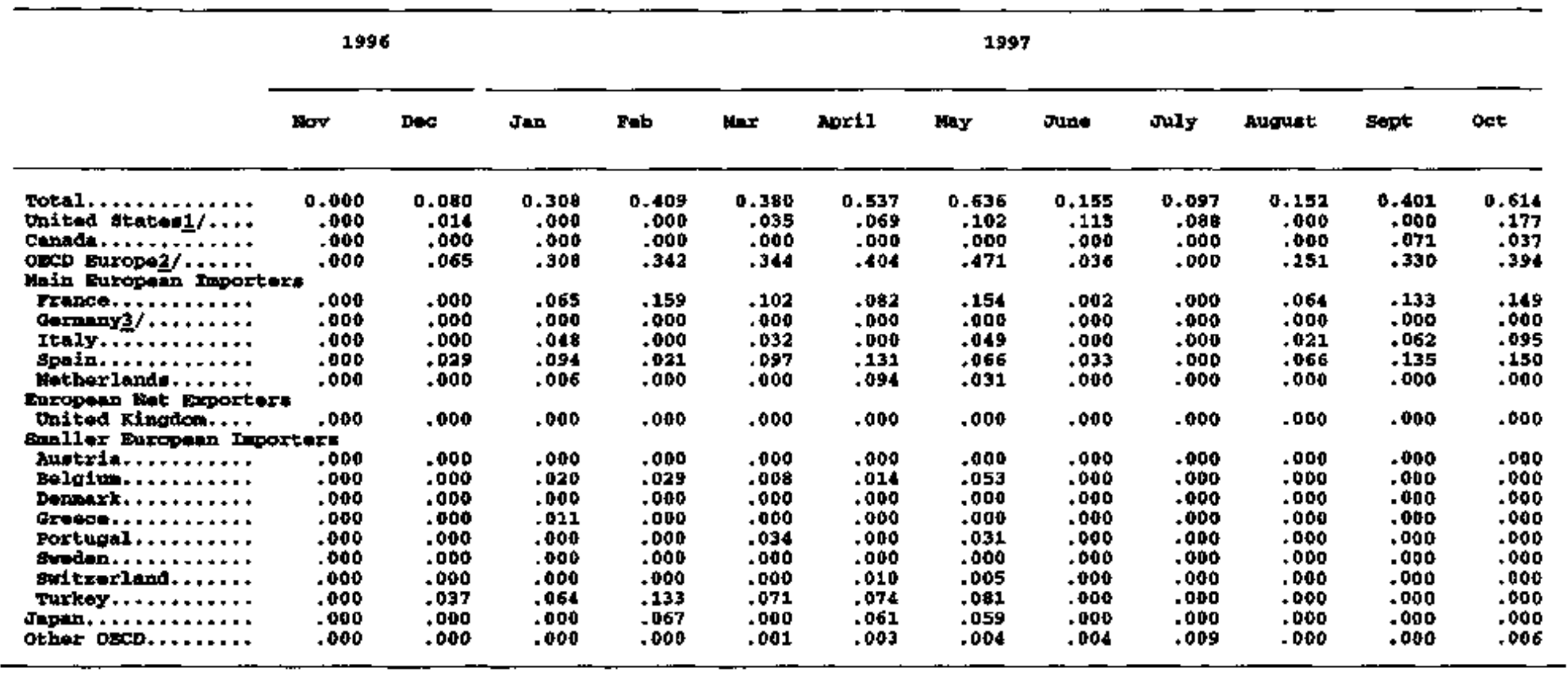

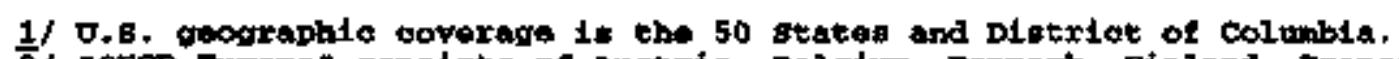

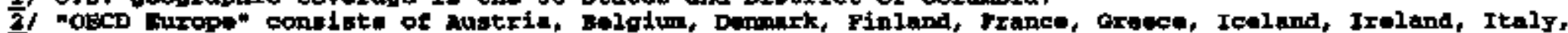

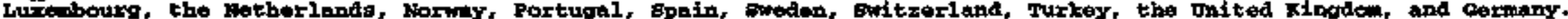

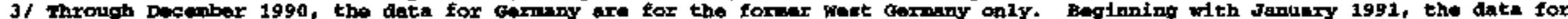

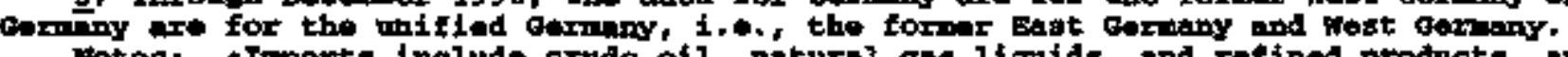

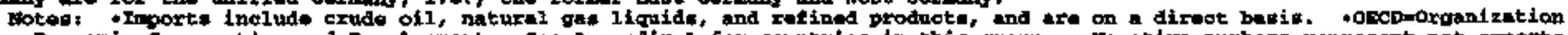

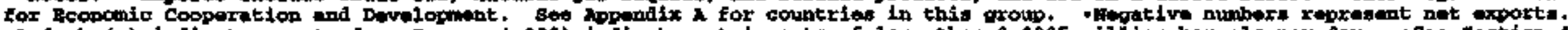

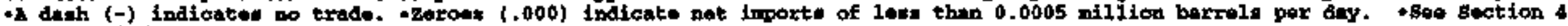
tor anmula inta.

gourcea: tot wad of seotion 3 . 
Table 3.14 CECD Inports from Kuwait (Wowt Rocont 12 Honths) (uillion Barriels pos Dey)

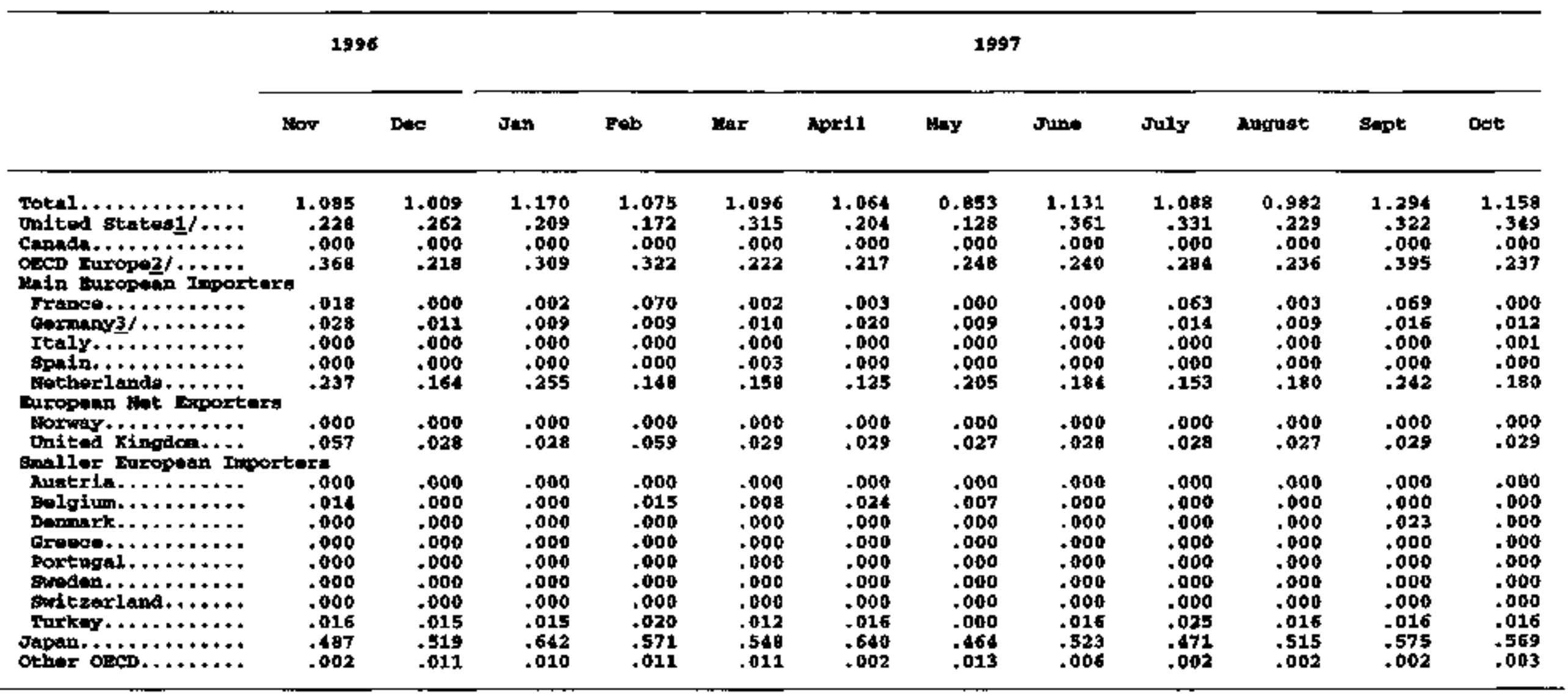

1/ U.8. Geographle coverage is the 30 states and pistrict of colubia.

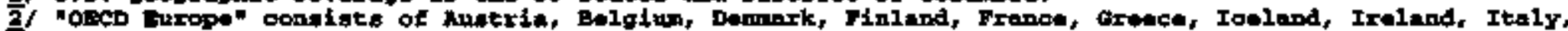

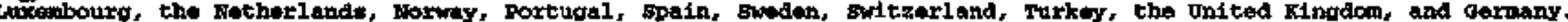

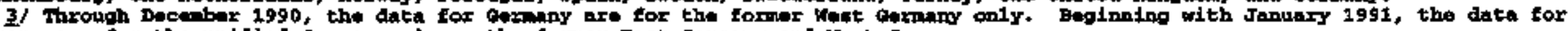

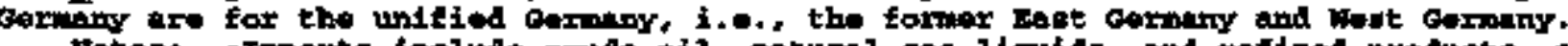

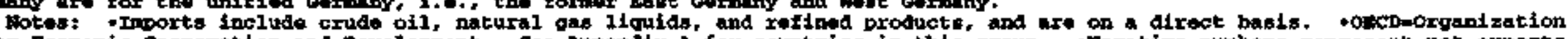

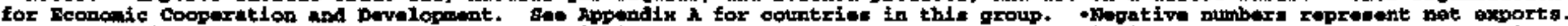

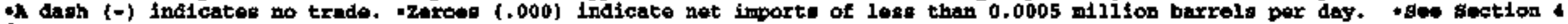
for annual data.

sources: see and of section 3. 


\section{Sources for Section 3 (International Oil Imports)}

\section{Oil Jmports}

United States: - Energy Information Administration (EIA), Petroleum Supoby Annual 1996 and Petroloum Supoly Monthly, Jannary 1998. Other OECD Countries: - Organizalion for Economic Cooperation and Development (OECD), Ouarterly Oil Stalistics and Enecgy Balances, various issties. - OECD, Motwily Oil Statistics data base, 1991-1997. 
4. Historical Oll Data Series $1970-1996$ 
Table 4.1a Forla Crude Oil Production (Including seave Condensatw), 1970-1996 (Thopeapd Barrele perr Day)

\begin{tabular}{|c|c|c|c|c|c|c|c|c|c|c|c|c|c|}
\hline & & Aloexis & $\begin{array}{l}\text { Indo- } \\
\text { neata }\end{array}$ & Iran & Irad & 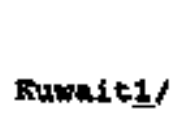 & Itbyra & Nigexis & Oatar & $\begin{array}{l}\text { sandi } \\
\text { Mrabley }\end{array}$ & $\begin{array}{l}\text { Wpited } \\
\text { Arab } \\
\text { E-icatea }\end{array}$ & $\begin{array}{l}\text { Vana- } \\
\text { zuele }\end{array}$ & $\begin{array}{l}\text { Total } \\
\text { OPEC }\end{array}$ \\
\hline $\begin{array}{l}1970 \\
1971 \\
1972 \\
1973 \\
1974 \\
1975 \\
1976 \\
1977 \\
1978 \\
1979 \\
1980 \\
1981 \\
1982 \\
1983 \\
1984 \\
1985 \\
1986 \\
1987 \\
1989 \\
1989 \\
1990 \\
1991 \\
1992 \\
1993 \\
1994 \\
2995 \\
1996\end{array}$ & 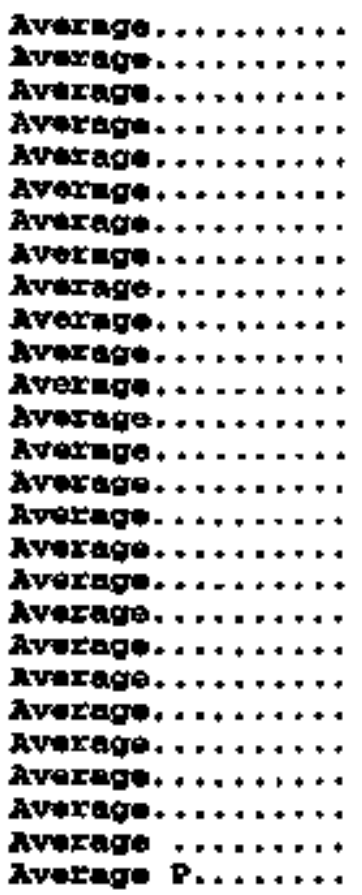 & $\begin{array}{r}1,029 \\
7,05 \\
1,062 \\
1,097 \\
1,009 \\
1,983 \\
1,159 \\
1,231 \\
1,224 \\
1,106 \\
1,002 \\
987 \\
1,018 \\
1,037 \\
1,045 \\
1,040 \\
1,095 \\
1,175 \\
1,230 \\
1,214 \\
1,160 \\
1,180 \\
1,202 \\
1,242\end{array}$ & $\begin{array}{l}1954 \\
1,092 \\
1,081 \\
1,339 \\
1,375 \\
1,307 \\
1,504 \\
1,606 \\
1,635 \\
1,591 \\
1,577 \\
1,605 \\
1,399 \\
1,343 \\
1,412 \\
1,325 \\
1,390 \\
1,343 \\
1,342 \\
1,409 \\
1,462 \\
1,592 \\
1,504 \\
1,511 \\
1,510 \\
1,503 \\
1,547\end{array}$ & $\begin{array}{l}3,829 \\
4,540 \\
5,023 \\
5,861 \\
6,022 \\
5,350 \\
5,8133 \\
5,663 \\
5,242 \\
3,160 \\
1,663 \\
1,380 \\
2,214 \\
2,440 \\
2,174 \\
2,250 \\
2,035 \\
2,298 \\
2,240 \\
2,810 \\
3,086 \\
3,312 \\
3,429 \\
3,540 \\
3,618 \\
3,643 \\
3,686\end{array}$ & $\begin{array}{r}1,549 \\
1,694 \\
1,666 \\
2,018 \\
1,971 \\
2,262 \\
2,015 \\
2,340 \\
2,563 \\
3,477 \\
2,514 \\
1,000 \\
1,012 \\
1,005 \\
1,209 \\
1,433 \\
1,690 \\
2,079 \\
2,685 \\
2,897 \\
2,040 \\
305 \\
425 \\
512 \\
553 \\
560 \\
584\end{array}$ & $\begin{array}{l}2,990 \\
3,197 \\
3,283 \\
3,020 \\
2,546 \\
2,084 \\
2,145 \\
1,969 \\
2,131 \\
2,500 \\
1,656 \\
1,125 \\
1,053 \\
1,157 \\
1,023 \\
1,419 \\
1,595 \\
1,492 \\
1,703 \\
1,175 \\
190 \\
1,056 \\
1,852 \\
2,025 \\
2,057 \\
2,062\end{array}$ & $\begin{array}{l}3,319 \\
2,761 \\
2,239 \\
2,173 \\
1,521 \\
1,480 \\
1,933 \\
2,063 \\
1,983 \\
2,092 \\
1,787 \\
1,140 \\
1,150 \\
1,105 \\
1,087 \\
1,059 \\
1,034 \\
1,972 \\
1,175 \\
1,150 \\
1,375 \\
1,463 \\
1,433 \\
1,361 \\
1,376 \\
1,390 \\
1,401\end{array}$ & $\begin{array}{l}1,083 \\
1,531 \\
1,016 \\
2,054 \\
2,255 \\
1,783 \\
2,067 \\
2,085 \\
1,897 \\
2,302 \\
2,055 \\
1,433 \\
1,295 \\
1,241 \\
1,389 \\
1,495 \\
1,467 \\
1,941 \\
1,450 \\
1,716 \\
1,010 \\
1,892 \\
1,943 \\
1,960 \\
1,931 \\
1,993 \\
2,108\end{array}$ & $\begin{array}{l}362 \\
431 \\
482 \\
570 \\
518 \\
438 \\
497 \\
445 \\
487 \\
508 \\
472 \\
405 \\
330 \\
295 \\
394 \\
301 \\
309 \\
293 \\
346 \\
390 \\
406 \\
395 \\
423 \\
613 \\
415 \\
493 \\
510\end{array}$ & $\begin{array}{l}3,799 \\
4,769 \\
6,016 \\
7,596 \\
8,480 \\
7,075 \\
8,577 \\
9,245 \\
8,301 \\
9,532 \\
9,900 \\
9,815 \\
6,483 \\
5,086 \\
1,663 \\
3,388 \\
4,870 \\
4,265 \\
5,015 \\
5,064 \\
6,410 \\
8,115 \\
6,332 \\
6,198 \\
6,120 \\
8,231 \\
6,218\end{array}$ & $\begin{array}{r}760 \\
1,060 \\
1,203 \\
1,533 \\
1,679 \\
1,664 \\
1,936 \\
1,999 \\
1,831 \\
1,831 \\
1,709 \\
1,474 \\
1,250 \\
1,149 \\
1,146 \\
1,193 \\
1,330 \\
1,541 \\
1,565 \\
1,860 \\
2,117 \\
2,386 \\
2,266 \\
2,159 \\
2,193 \\
2,279 \\
2,279\end{array}$ & $\begin{array}{l}3,708 \\
3,549 \\
3,210 \\
3,366 \\
2,976 \\
2,346 \\
2,294 \\
2,239 \\
2,165 \\
2,356 \\
2,168 \\
2,102 \\
1,895 \\
1,801 \\
1,799 \\
1,677 \\
1,767 \\
1,752 \\
1,903 \\
1,907 \\
2,137 \\
2,375 \\
2,371 \\
2,450 \\
2,599 \\
2,750 \\
3,053\end{array}$ & $\begin{array}{l}23,301 \\
25,209 \\
26,891 \\
30,629 \\
30,351 \\
26,771 \\
30,327 \\
30,893 \\
29,464 \\
30,581 \\
26,606 \\
22,481 \\
18,778 \\
17,497 \\
17,442 \\
16,181 \\
18,275 \\
18,517 \\
20,321 \\
22,071 \\
23,195 \\
23,275 \\
24,398 \\
25,119 \\
25,510 \\
26,092 \\
26,769\end{array}$ \\
\hline
\end{tabular}

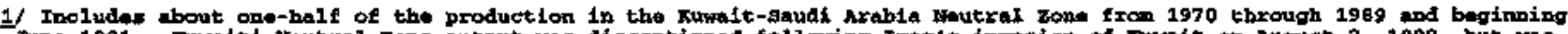

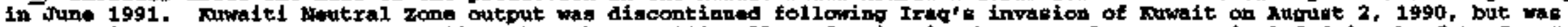

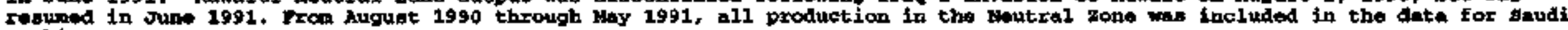
Arable.

Paprelininary.

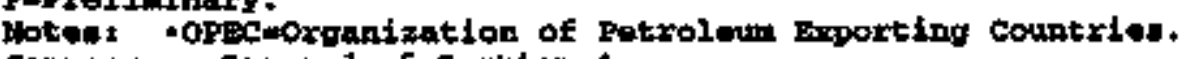

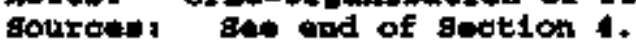


Table 4.1b For1a crode oll Production (Including Inase Condennate), 1970-1996 (Thousand Barrele Dor Dny)

\begin{tabular}{|c|c|c|c|c|c|c|c|c|c|c|c|c|}
\hline & & Notway & $\begin{array}{l}\text { Jnited } \\
\text { ningdera }\end{array}$ & $\begin{array}{l}\text { North } \\
\text { steal } /\end{array}$ & Angola & $\begin{array}{l}\text { arpen- } \\
\text { tina }\end{array}$ & $\begin{array}{c}\text { Auntra- } \\
\text { lia }\end{array}$ & Arazil & Connde & chint & Colcalbia & Eoundor \\
\hline $\begin{array}{l}1970 \\
1971 \\
1972 \\
1973 \\
1974 \\
1975 \\
1976 \\
1977 \\
1978 \\
1979 \\
1980 \\
1981 \\
1982 \\
1983 \\
1984 \\
1985 \\
1986 \\
1987 \\
1980 \\
1969 \\
1990 \\
1991 \\
1992 \\
1993 \\
1994 \\
1995 \\
1996\end{array}$ & 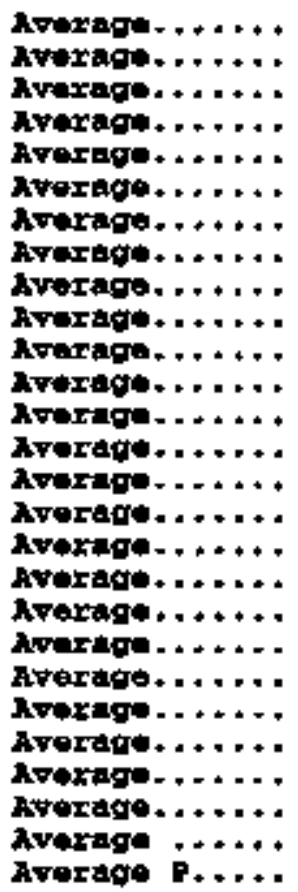 & $\begin{array}{r}9 \\
63 \\
32 \\
35 \\
189 \\
279 \\
280 \\
356 \\
403 \\
528 \\
501 \\
520 \\
614 \\
697 \\
788 \\
970 \\
1,027 \\
1,158 \\
1,554 \\
1,704 \\
1,690 \\
2,229 \\
2,350 \\
2,521 \\
3,768 \\
3,104\end{array}$ & $\begin{array}{r}2 \\
2 \\
2 \\
2 \\
12 \\
245 \\
768 \\
1,082 \\
1,568 \\
1,622 \\
1,811 \\
3,065 \\
2,291 \\
2,480 \\
2,530 \\
2,539 \\
2,406 \\
2,232 \\
1,802 \\
1,920 \\
1,797 \\
1,925 \\
1,915 \\
2,375 \\
2,409 \\
2,568\end{array}$ & $\begin{array}{r}0 \\
6 \\
35 \\
36 \\
202 \\
525 \\
1,055 \\
1,446 \\
1,976 \\
2,151 \\
2,322 \\
2,617 \\
2,965 \\
3,250 \\
3,417 \\
3,547 \\
3,584 \\
3,540 \\
3,515 \\
3,667 \\
3,811 \\
4,182 \\
4,103 \\
5,058 \\
5,100 \\
5,822\end{array}$ & $\begin{array}{r}112 \\
93 \\
140 \\
162 \\
169 \\
165 \\
108 \\
194 \\
131 \\
147 \\
150 \\
130 \\
122 \\
177 \\
208 \\
231 \\
282 \\
360 \\
452 \\
455 \\
475 \\
500 \\
526 \\
509 \\
536 \\
646 \\
709\end{array}$ & $\begin{array}{l}393 \\
423 \\
433 \\
420 \\
414 \\
395 \\
398 \\
431 \\
453 \\
473 \\
491 \\
496 \\
491 \\
491 \\
470 \\
460 \\
434 \\
428 \\
449 \\
460 \\
489 \\
495 \\
559 \\
594 \\
650 \\
715 \\
756\end{array}$ & $\begin{array}{l}178 \\
309 \\
327 \\
369 \\
388 \\
409 \\
426 \\
431 \\
432 \\
441 \\
380 \\
394 \\
370 \\
416 \\
492 \\
575 \\
520 \\
547 \\
539 \\
490 \\
575 \\
545 \\
535 \\
503 \\
536 \\
562 \\
570\end{array}$ & $\begin{array}{l}167 \\
174 \\
167 \\
169 \\
176 \\
177 \\
167 \\
161 \\
160 \\
166 \\
182 \\
213 \\
260 \\
339 \\
475 \\
564 \\
572 \\
566 \\
551 \\
596 \\
631 \\
630 \\
626 \\
613 \\
671 \\
595 \\
795\end{array}$ & $\begin{array}{l}1,263 \\
1,348 \\
1,532 \\
1,798 \\
1,551 \\
1,430 \\
1,314 \\
1,321 \\
1,316 \\
1,500 \\
1,435 \\
1,295 \\
1,271 \\
1,356 \\
1,438 \\
1,471 \\
1,474 \\
1,535 \\
1,616 \\
1,560 \\
1,553 \\
1,549 \\
1,605 \\
1,679 \\
1,746 \\
1,805 \\
1,823\end{array}$ & $\begin{array}{r}600 \\
780 \\
900 \\
1,094 \\
1,315 \\
1,490 \\
1,670 \\
1,974 \\
2,082 \\
2,132 \\
2,114 \\
2,012 \\
2,045 \\
2,120 \\
2,296 \\
2,505 \\
2,620 \\
2,690 \\
2,730 \\
2,757 \\
2,774 \\
2,035 \\
2,645 \\
2,890 \\
2,939 \\
2,990 \\
3,131\end{array}$ & $\begin{array}{l}219 \\
214 \\
196 \\
184 \\
168 \\
157 \\
146 \\
138 \\
131 \\
124 \\
126 \\
125 \\
141 \\
152 \\
168 \\
176 \\
305 \\
385 \\
379 \\
403 \\
440 \\
419 \\
433 \\
456 \\
450 \\
585 \\
623\end{array}$ & $\begin{array}{r}4 \\
7 \\
709 \\
177 \\
161 \\
198 \\
183 \\
202 \\
214 \\
204 \\
211 \\
211 \\
237 \\
258 \\
281 \\
293 \\
174 \\
302 \\
279 \\
205 \\
299 \\
321 \\
344 \\
365 \\
392 \\
387\end{array}$ \\
\hline
\end{tabular}

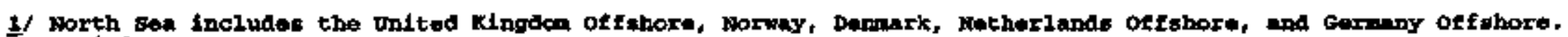
Faprelininary.

Bources: see and of section 4 . 


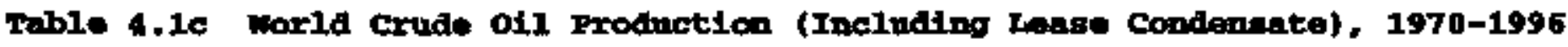

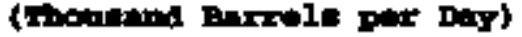

\begin{tabular}{|c|c|c|c|c|c|c|c|c|c|c|c|c|c|}
\hline & & rogpt & Gabod & Inalia & Malesosa & Ionstoo & Cran & Rasaln & $\begin{array}{l}\text { Fom.s. } \\
\text { O.t.6.R. }\end{array}$ & byta & $\begin{array}{l}\text { onited } \\
\text { stater }\end{array}$ & others 1 & Torla \\
\hline $\begin{array}{l}1970 \\
1971 \\
1972 \\
1973 \\
1974 \\
1975 \\
1976 \\
1977 \\
1978 \\
1979 \\
1980 \\
1981 \\
1982 \\
1983 \\
1984 \\
1985 \\
1986 \\
1987 \\
1998 \\
1989 \\
1990 \\
1991 \\
1992 \\
1993 \\
1994 \\
1995 \\
1996\end{array}$ & 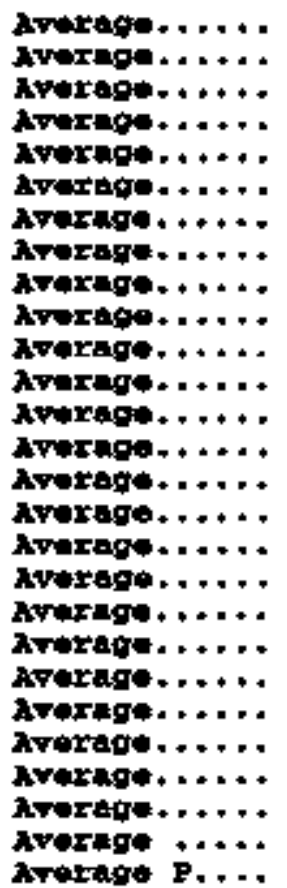 & $\begin{array}{l}326 \\
293 \\
231 \\
165 \\
150 \\
235 \\
330 \\
415 \\
485 \\
525 \\
595 \\
598 \\
670 \\
727 \\
822 \\
887 \\
813 \\
896 \\
849 \\
965 \\
873 \\
874 \\
681 \\
690 \\
896 \\
920 \\
922\end{array}$ & $\begin{array}{l}109 \\
115 \\
135 \\
150 \\
202 \\
223 \\
223 \\
222 \\
209 \\
203 \\
175 \\
151 \\
156 \\
157 \\
157 \\
172 \\
166 \\
155 \\
159 \\
208 \\
270 \\
294 \\
298 \\
313 \\
329 \\
345 \\
369\end{array}$ & $\begin{array}{l}146 \\
143 \\
156 \\
147 \\
156 \\
165 \\
175 \\
199 \\
226 \\
245 \\
182 \\
329 \\
390 \\
180 \\
519 \\
620 \\
630 \\
609 \\
635 \\
700 \\
660 \\
615 \\
561 \\
534 \\
590 \\
709 \\
651\end{array}$ & $\begin{array}{l}18 \\
69 \\
93 \\
91 \\
91 \\
98 \\
165 \\
184 \\
217 \\
283 \\
283 \\
264 \\
306 \\
365 \\
440 \\
440 \\
504 \\
497 \\
540 \\
595 \\
619 \\
646 \\
653 \\
640 \\
645 \\
682 \\
695\end{array}$ & $\begin{array}{r}467 \\
486 \\
505 \\
465 \\
571 \\
705 \\
831 \\
981 \\
1,209 \\
1,461 \\
1,936 \\
2,313 \\
2,748 \\
2,689 \\
3,780 \\
2,745 \\
2,435 \\
2,548 \\
2,512 \\
2,520 \\
2,553 \\
2,690 \\
2,669 \\
2,673 \\
2,695 \\
2,618 \\
2,655\end{array}$ & $\begin{array}{l}332 \\
994 \\
289 \\
299 \\
290 \\
943 \\
366 \\
341 \\
315 \\
295 \\
282 \\
319 \\
324 \\
375 \\
414 \\
498 \\
560 \\
582 \\
617 \\
641 \\
585 \\
700 \\
740 \\
776 \\
810 \\
851 \\
683\end{array}$ & 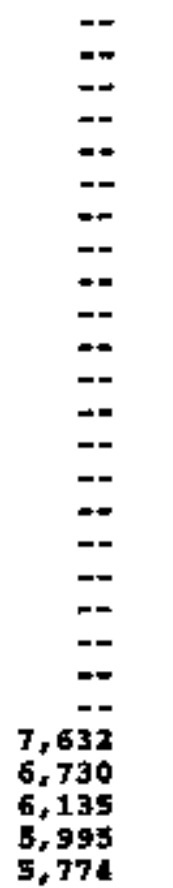 & $\begin{array}{r}6,985 \\
7,475 \\
7,990 \\
8,924 \\
8,912 \\
9,523 \\
10,060 \\
10,603 \\
11,105 \\
11,364 \\
11,706 \\
11,950 \\
11,912 \\
11,972 \\
11,961 \\
11,585 \\
11,995 \\
12,050 \\
12,053 \\
11,715 \\
10,975 \\
9,992 \\
-- \\
-- \\
-2 \\
-- \\
-2\end{array}$ & $\begin{array}{l}80 \\
100 \\
124 \\
105 \\
124 \\
170 \\
175 \\
188 \\
170 \\
166 \\
164 \\
166 \\
160 \\
167 \\
170 \\
178 \\
194 \\
230 \\
265 \\
340 \\
380 \\
492 \\
481 \\
554 \\
560 \\
610 \\
604\end{array}$ & $\begin{array}{l}9,637 \\
9,463 \\
9,411 \\
9,208 \\
9,774 \\
9,375 \\
0,132 \\
9,245 \\
8,707 \\
9,592 \\
8,597 \\
8,572 \\
8,649 \\
9,680 \\
9,879 \\
8,971 \\
9,690 \\
8,349 \\
6,140 \\
7,613 \\
7,355 \\
7,417 \\
7,171 \\
6,947 \\
6,662 \\
6,560 \\
6,465\end{array}$ & $\begin{array}{l}1,529 \\
1,518 \\
1,592 \\
1,667 \\
1,711 \\
1,636 \\
1,620 \\
1,655 \\
1,706 \\
1,921 \\
1,842 \\
1,859 \\
1,852 \\
1,946 \\
2,015 \\
2,124 \\
3,166 \\
2,120 \\
2,235 \\
1,249 \\
2,253 \\
2,274 \\
3,233 \\
3,290 \\
3,393 \\
3,423 \\
1,520\end{array}$ & $\begin{array}{l}45,886 \\
48,518 \\
51,138 \\
55,679 \\
55,716 \\
52,928 \\
97,344 \\
59,707 \\
60,158 \\
62,674 \\
59,600 \\
56,076 \\
53,481 \\
53,256 \\
51,489 \\
53,982 \\
56,227 \\
56,666 \\
58,737 \\
59,863 \\
60,566 \\
60,207 \\
60,216 \\
60,247 \\
61,003 \\
62,466 \\
63,972\end{array}$ \\
\hline
\end{tabular}

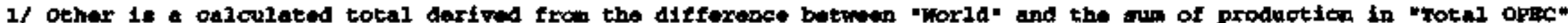

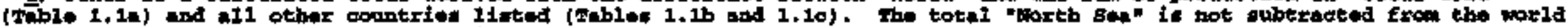

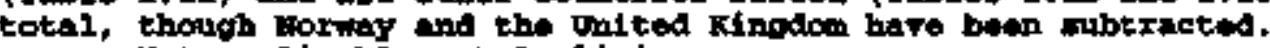

- = Hot applicable. Peprelintary.

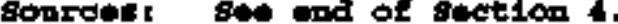


Table 4.2 OpEc Crude oll Production (Fonluding Condensete), 1980-1996 (rhounand Barrol: por Day)

\begin{tabular}{|c|c|c|c|c|c|c|c|c|c|c|c|c|c|}
\hline & & 100하료 & $\begin{array}{l}\text { Indo- } \\
\text { nt:ilid }\end{array}$ & Iran & Ixtax & Krwa1t $1 /$ & Libye & Nigerla & Gatax & $\begin{array}{l}\text { gandi } \\
\text { arabial/ }\end{array}$ & $\begin{array}{l}\text { trattod } \\
\text { Arab } \\
\text { rivatess }\end{array}$ & $\begin{array}{l}\text { Veno- } \\
\text { ztola }\end{array}$ & $\begin{array}{l}\text { Toted } \\
\text { OPBC }\end{array}$ \\
\hline $\begin{array}{l}1990 \\
1981 \\
1982 \\
1983 \\
1984 \\
1985 \\
1985 \\
1987 \\
1980 \\
1989 \\
1990 \\
1991 \\
1992 \\
1993 \\
1994 \\
1995 \\
1996\end{array}$ & 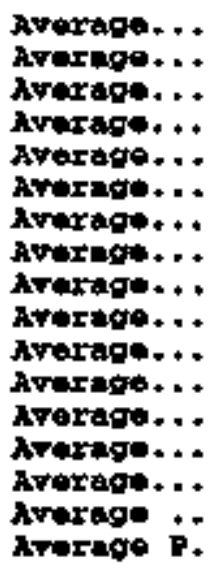 & $\begin{array}{r}1,006 \\
802 \\
712 \\
640 \\
694 \\
702 \\
600 \\
648 \\
640 \\
690 \\
765 \\
800 \\
772 \\
747 \\
750 \\
767 \\
612\end{array}$ & $\begin{array}{l}1,497 \\
1,51,3 \\
1,247 \\
1,245 \\
1,279 \\
1,181 \\
1,257 \\
1,189 \\
1,177 \\
1,231 \\
1,291 \\
1,410 \\
1,326 \\
1,327 \\
1,334 \\
1,343 \\
1,367\end{array}$ & $\begin{array}{l}1,662 \\
1,380 \\
2,214 \\
2,440 \\
2,174 \\
2,250 \\
2,035 \\
2,208 \\
2,240 \\
2,810 \\
3,080 \\
3,312 \\
3,429 \\
3,540 \\
3,600 \\
3,608 \\
3,651\end{array}$ & $\begin{array}{r}2,514 \\
1,000 \\
1,012 \\
1,005 \\
1,209 \\
1,433 \\
1,690 \\
2,079 \\
2,685 \\
2,897 \\
2,040 \\
305 \\
125 \\
512 \\
548 \\
550 \\
579\end{array}$ & $\begin{array}{r}1,655 \\
1,125 \\
1,023 \\
1,064 \\
1,157 \\
1,023 \\
1,019 \\
1,585 \\
1,492 \\
1,763 \\
1,175 \\
190 \\
1,058 \\
1,052 \\
2,034 \\
2,057 \\
2,062\end{array}$ & $\begin{array}{l}1,787 \\
1,140 \\
1,150 \\
1,105 \\
1,087 \\
1,059 \\
1,034 \\
972 \\
1,175 \\
1,150 \\
1,375 \\
1,483 \\
1,433 \\
1,361 \\
1,378 \\
1,390 \\
1,401\end{array}$ & $\begin{array}{l}2,045 \\
1,423 \\
1,285 \\
1,231 \\
1,368 \\
1,475 \\
1,447 \\
1,291 \\
1,440 \\
1,656 \\
1,755 \\
1,632 \\
1,820 \\
1,835 \\
1,897 \\
1,676 \\
2,051\end{array}$ & $\begin{array}{l}472 \\
405 \\
330 \\
295 \\
394 \\
301 \\
300 \\
293 \\
346 \\
380 \\
406 \\
395 \\
423 \\
413 \\
409 \\
449 \\
475\end{array}$ & $\begin{array}{l}9,900 \\
9,815 \\
6,403 \\
5,096 \\
4,663 \\
3,388 \\
4,870 \\
4,265 \\
5,086 \\
5,064 \\
6,410 \\
8,115 \\
8,332 \\
9,198 \\
8,147 \\
8,231 \\
8,218\end{array}$ & $\begin{array}{l}1,704 \\
1,469 \\
1,213 \\
1,109 \\
1,092 \\
1,133 \\
1,270 \\
1,441 \\
1,465 \\
1,783 \\
2,066 \\
2,326 \\
2,206 \\
2,109 \\
2,183 \\
2,197 \\
2,198\end{array}$ & $\begin{array}{l}2,147 \\
2,083 \\
1,078 \\
1,768 \\
1,689 \\
1,558 \\
1,645 \\
1,587 \\
1,715 \\
1,747 \\
2,097 \\
2,338 \\
2,334 \\
2,410 \\
2,547 \\
2,710 \\
3,013\end{array}$ & $\begin{array}{l}26,390 \\
20,155 \\
18,349 \\
16,996 \\
16,805 \\
15,503 \\
17,575 \\
17,648 \\
19,421 \\
21,201 \\
22,458 \\
22,506 \\
23,558 \\
24,304 \\
24,612 \\
25,179 \\
25,826\end{array}$ \\
\hline
\end{tabular}

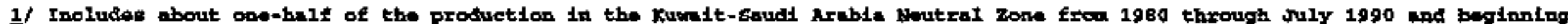

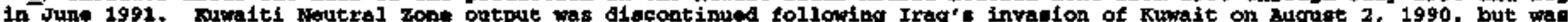

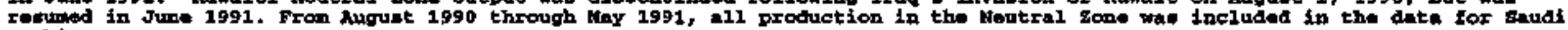
arabia.

PaPrelisinary.

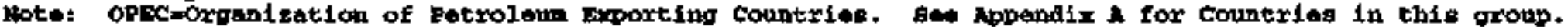

sourcert sto and of section to 
Fable 4.3 Worla naturel ens plant riguids prohnction, 1970-1996

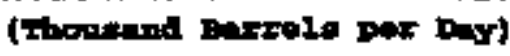

\begin{tabular}{|c|c|c|c|c|c|c|c|c|c|c|c|c|c|}
\hline & & & $\cos \leqslant$ & Conndin & Mond oo & $\begin{array}{l}\text { anudt } \\
\text { rrabst }\end{array}$ & Rusata & $\begin{array}{l}\text { Former } \\
\text { dust }\end{array}$ & $\begin{array}{l}\text { Gatted } \\
\text { statediy }\end{array}$ & Parting & OAPEC3/ & oppxeㄱ & Mor1d \\
\hline $\begin{array}{l}1970 \\
1971 \\
1972 \\
1973 \\
1974 \\
1975 \\
1976 \\
1977 \\
1979 \\
1979 \\
1980 \\
1981 \\
1982 \\
1983 \\
1984 \\
1985 \\
1986 \\
1987 \\
1988 \\
1989 \\
1990 \\
1991 \\
1992 \\
1993 \\
1994 \\
1995 \\
1996\end{array}$ & 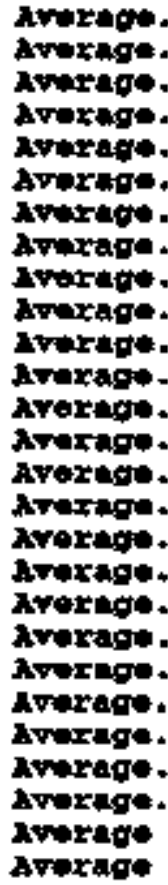 & 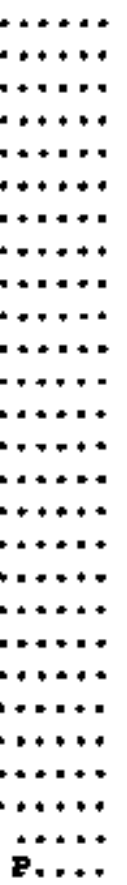 & $\begin{array}{r}3 \\
4 \\
9 \\
12 \\
20 \\
24 \\
19 \\
25 \\
30 \\
36 \\
49 \\
50 \\
56 \\
105 \\
120 \\
120 \\
140 \\
120 \\
130 \\
130 \\
140 \\
140 \\
145 \\
140 \\
145 \\
145\end{array}$ & $\begin{array}{l}200 \\
234 \\
293 \\
314 \\
314 \\
309 \\
289 \\
290 \\
281 \\
331 \\
331 \\
330 \\
318 \\
309 \\
336 \\
337 \\
329 \\
367 \\
381 \\
410 \\
426 \\
431 \\
460 \\
506 \\
539 \\
581 \\
599\end{array}$ & $\begin{array}{r}58 \\
59 \\
61 \\
75 \\
80 \\
80 \\
95 \\
105 \\
115 \\
150 \\
193 \\
241 \\
255 \\
265 \\
257 \\
271 \\
352 \\
339 \\
370 \\
394 \\
428 \\
457 \\
454 \\
459 \\
461 \\
447 \\
423\end{array}$ & $\begin{array}{l}50 \\
50 \\
55 \\
90 \\
130 \\
140 \\
185 \\
215 \\
250 \\
303 \\
369 \\
433 \\
430 \\
330 \\
355 \\
375 \\
385 \\
418 \\
499 \\
503 \\
620 \\
680 \\
713 \\
704 \\
698 \\
701 \\
697\end{array}$ & 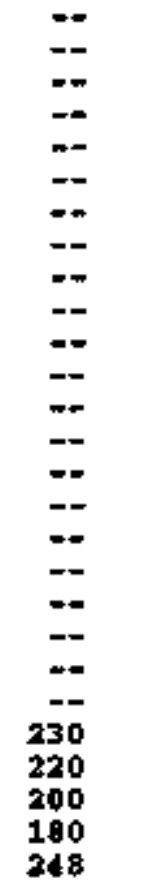 & $\begin{array}{l}120 \\
135 \\
155 \\
270 \\
190 \\
205 \\
220 \\
235 \\
255 \\
270 \\
285 \\
300 \\
315 \\
330 \\
340 \\
350 \\
140 \\
430 \\
450 \\
425 \\
425 \\
420 \\
-2 \\
-= \\
=- \\
-= \\
=\end{array}$ & $\begin{array}{l}1,660 \\
1,693 \\
1,744 \\
1,738 \\
1,689 \\
1,633 \\
1,603 \\
1,618 \\
1,567 \\
1,584 \\
1,573 \\
1,609 \\
1,550 \\
1,559 \\
1,630 \\
1,609 \\
1,551 \\
1,595 \\
1,625 \\
1,546 \\
1,599 \\
1,659 \\
1,697 \\
1,736 \\
1,727 \\
1,762 \\
1,830\end{array}$ & $\begin{array}{r}119 \\
138 \\
155 \\
191 \\
231 \\
246 \\
291 \\
335 \\
410 \\
458 \\
534 \\
599 \\
612 \\
552 \\
604 \\
645 \\
700 \\
721 \\
908 \\
851 \\
930 \\
931 \\
1,003 \\
\pm, 040 \\
1,071 \\
1,106 \\
1,082\end{array}$ & $\begin{array}{r}137 \\
147 \\
159 \\
196 \\
219 \\
252 \\
313 \\
365 \\
447 \\
535 \\
618 \\
691 \\
721 \\
655 \\
761 \\
608 \\
860 \\
900 \\
979 \\
1,041 \\
1,107 \\
1,113 \\
1,185 \\
1,238 \\
1,267 \\
1,301 \\
1,274\end{array}$ & $\begin{array}{r}205 \\
243 \\
275 \\
324 \\
547 \\
372 \\
442 \\
482 \\
566 \\
637 \\
732 \\
825 \\
642 \\
780 \\
069 \\
892 \\
969 \\
1,006 \\
1,077 \\
1,188 \\
1,281 \\
1,299 \\
1,364 \\
1,435 \\
1,465 \\
1,506 \\
1,452\end{array}$ & $\begin{array}{l}2,383 \\
2,508 \\
2,676 \\
2,795 \\
2,799 \\
2,790 \\
2,665 \\
2,992 \\
3,078 \\
3,285 \\
3,444 \\
3,625 \\
3,626 \\
3,635 \\
3,869 \\
3,938 \\
4,150 \\
4,279 \\
4,481 \\
4,502 \\
4,632 \\
4,827 \\
4,973 \\
5,169 \\
5,282 \\
5,474 \\
5,625\end{array}$ \\
\hline
\end{tabular}

t/ U.\$. geopraphte coverage is the 50 atnted and the Diftriet of columbit.

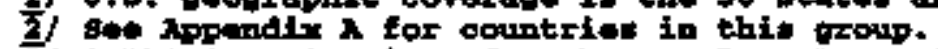

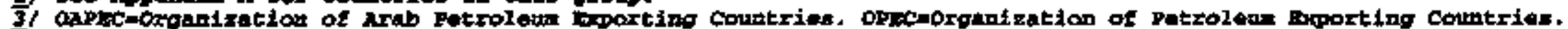

on lpowdix A for conntrien in thede groubs.

peproliminary.

sourens: seve and of ention 4 . 
Table 4.4 Forld 011 supply, 1970-1996

(rhousand Barroll par Day)

Onited

araing

AxPEC $\underline{3}$

opsc3/

Wor1d

1970 Ararage. . . . . . . . . .

1971 kverage. . . . . . . . . . .

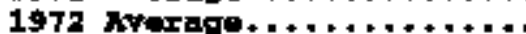

1973 Average...........

197 A Areriege. . . . . . . . . . .

1975 Arerage.$\ldots \ldots \ldots \ldots \ldots \ldots$

1976 hvarige. ............

1977 Avaraute. . . . . . . . .

1978 Aphritge. . . . . . . . . .

1979 Averapt. $\ldots \ldots \ldots \ldots \ldots \ldots$

1980 Averege. . . . . . . .

1982 Average. . . . . . . . .

1942 nvernge. . . . . . . . . .

1983 Averaute. . . . . . . . . . .

1984 dverrage............

1985 svorage . . . . . . . . . . .

1986 svwrage. . . . . . . . . .

1987 Average. . . . . . . . . . .

1988 Avorato . . . . . . . . .

1989 avernot. . . . . . . . . .

1990 avarage.............

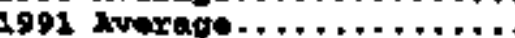

1992 Avernge. . . . . . . . . .

1993 Averege. . . . . . . . . .

1994 nverage. . . . . . . . . . .

1995 avarege $\ldots \ldots \ldots \ldots \ldots \ldots$

1996 Average P..........

Stateril

(2)

13,541

15,940

11,673

11,601

11,428

10,978

17,734

19,530

10,251

10.47

21,847

10,820

10,707

10,809

22,098

21,569

18,541

10,739

15,894

12,815

10,708

11,107

11,433

11,192

10,320

12,41

10,648 12,859

14,310

10,473

9,800

15,733

$\begin{array}{ll}9,677 & 16,249\end{array}$

15,764

9,768

17,790

9,602

9,413

9,400

17,792

18,072

$9,445 \quad 18,491$

$14,57 t$

15,427

16, 151

18,656

19,399

16,831

19,563

20,372

19,819

22, 575

20,715

17,619

13,786

12,416

12,619

11,498

13,653

13,992

15,662

$16,65 B$

17,232

16,747

17.971

18,505

10,745

19,192

19,243

$\begin{array}{ll}23,567 & 48,986 \\ 25,514 & 51,766 \\ 27,228 & 54,574 \\ 31,415 & 59,300 \\ 30,762 & 59,391 \\ 27,218 & 56,511 \\ 30,841 & 61,121 \\ 31,443 & 63,665 \\ 30,120 & 64,225 \\ 31,300 & 66,973 \\ 27,419 & 64,253 \\ 23,393 & 60,763 \\ 19,702 & 58,226 \\ 18,352 & 58,056 \\ 18,389 & 59,645 \\ 17,151 & 59,264 \\ 19,310 & 61,771 \\ 19,589 & 62,429 \\ 21,465 & 64,707 \\ 23,323 & 65,994 \\ 24,536 & 66,756 \\ 24,625 & 66,634 \\ 25,618 & 66,937 \\ 26,610 & 67,345 \\ 27,031 & 68,218 \\ 27,654 & 69,916 \\ 28,277 & 71,739\end{array}$

1) U.s. geographio coverage 1/ the 50 gtates and the Distriot of colvbin.

2/ 900 Appondix $\lambda$ tor countrites in tbis groug.

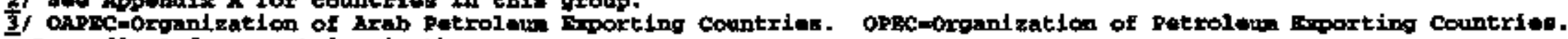

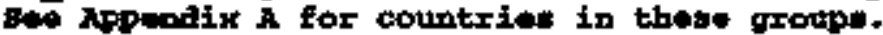

Peprelinimaxy.

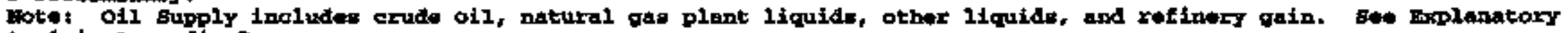
Wote 1 in appondix 1 .

acurces: Ene and or section 4. 


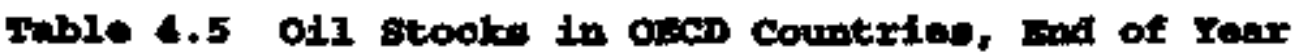

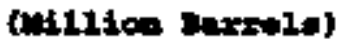

\begin{tabular}{|c|c|c|c|c|c|c|c|c|c|c|c|}
\hline & & France & $I t=1 y$ & Anringy & $\begin{array}{l}\text { Onlted } \\
\text { rifnotem }\end{array}$ & oxpoper & $\begin{array}{l}\text { Onjtend } \\
\text { otraten: } 1 /\end{array}$ & Ganada & Japan & otherey & $\begin{array}{l}\text { Total } \\
\text { oftep }\end{array}$ \\
\hline $\begin{array}{l}1973 \\
1974 \\
1975 \\
1976 \\
1977 \\
1979 \\
1979 \\
1980 \\
1981 \\
1987 \\
1993 \\
1994 \\
1985 \\
1916 \\
1987 \\
1988 \\
1989 \\
1990 \\
1991 \\
1992 \\
1993 \\
1994 \\
1995 \\
1996\end{array}$ & 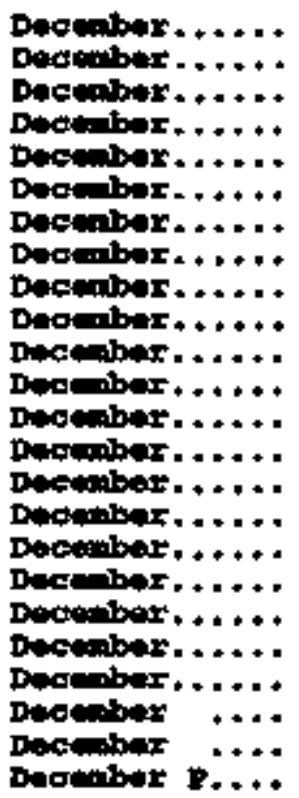 & $\begin{array}{l}201 \\
249 \\
225 \\
234 \\
239 \\
201 \\
226 \\
243 \\
214 \\
193 \\
153 \\
153 \\
139 \\
127 \\
127 \\
140 \\
138 \\
140 \\
153 \\
146 \\
158 \\
158 \\
159 \\
156\end{array}$ & $\begin{array}{l}152 \\
167 \\
143 \\
143 \\
161 \\
154 \\
163 \\
170 \\
167 \\
179 \\
149 \\
159 \\
157 \\
155 \\
169 \\
155 \\
164 \\
172 \\
160 \\
174 \\
163 \\
161 \\
162 \\
152\end{array}$ & $\begin{array}{l}181 \\
213 \\
187 \\
208 \\
225 \\
239 \\
272 \\
319 \\
297 \\
372 \\
249 \\
239 \\
233 \\
252 \\
259 \\
266 \\
271 \\
265 \\
268 \\
310 \\
309 \\
312 \\
301 \\
300\end{array}$ & $\begin{array}{l}156 \\
191 \\
165 \\
165 \\
148 \\
157 \\
169 \\
168 \\
113 \\
125 \\
118 \\
117 \\
123 \\
124 \\
121 \\
11.2 \\
118 \\
112 \\
119 \\
113 \\
118 \\
115 \\
107 \\
108\end{array}$ & $\begin{array}{l}1,070 \\
1,227 \\
1,154 \\
1,205 \\
1,269 \\
1,219 \\
1,353 \\
1,464 \\
1,337 \\
1,259 \\
1,113 \\
1,130 \\
1,092 \\
1,133 \\
1,130 \\
1,118 \\
1,133 \\
1,163 \\
1,181 \\
1,219 \\
1,221 \\
1,240 \\
1,228 \\
1,256\end{array}$ & $\begin{array}{l}1,009 \\
1,074 \\
1,133 \\
1,112 \\
1,312 \\
1,278 \\
1,341 \\
1,397 \\
1,494 \\
1,430 \\
1,454 \\
1,556 \\
1,519 \\
1,593 \\
1,607 \\
1,597 \\
1,591 \\
1,621 \\
1,617 \\
1,592 \\
1,647 \\
1,653 \\
1,563 \\
1,507\end{array}$ & $\begin{array}{l}140 \\
145 \\
174 \\
153 \\
167 \\
144 \\
150 \\
164 \\
161 \\
136 \\
121 \\
128 \\
113 \\
111 \\
126 \\
116 \\
114 \\
121 \\
119 \\
107 \\
105 \\
119 \\
109 \\
203\end{array}$ & $\begin{array}{l}303 \\
370 \\
375 \\
380 \\
409 \\
413 \\
460 \\
495 \\
482 \\
486 \\
670 \\
479 \\
494 \\
509 \\
540 \\
530 \\
577 \\
590 \\
606 \\
603 \\
610 \\
645 \\
630 \\
651\end{array}$ & $\begin{array}{l}67 \\
64 \\
67 \\
68 \\
68 \\
68 \\
75 \\
72 \\
67 \\
69 \\
68 \\
69 \\
66 \\
72 \\
71 \\
71 \\
71 \\
73 \\
65 \\
67 \\
69 \\
69 \\
71 \\
74\end{array}$ & $\begin{array}{l}2,588 \\
3,080 \\
2,903 \\
7,918 \\
3,224 \\
3,122 \\
3,379 \\
3,587 \\
3,531 \\
3,376 \\
3,255 \\
3,362 \\
3,284 \\
3,418 \\
3,474 \\
3,440 \\
3,476 \\
3,568 \\
3,588 \\
3,586 \\
3,661 \\
3,726 \\
3,601 \\
3,591\end{array}$ \\
\hline
\end{tabular}

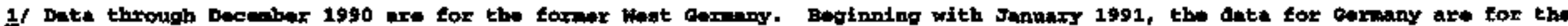

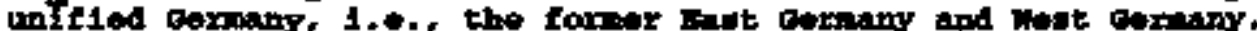

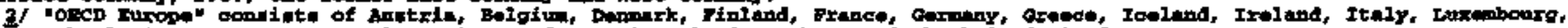

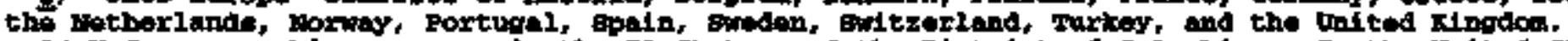

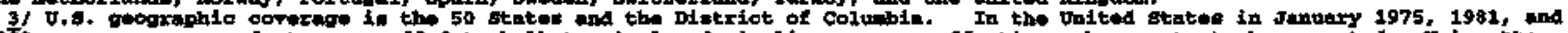

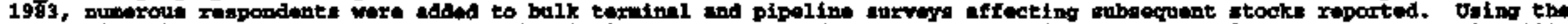

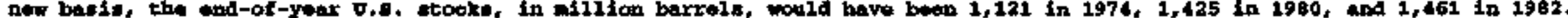

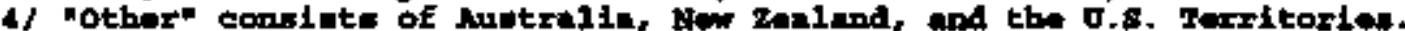

Prepritining.

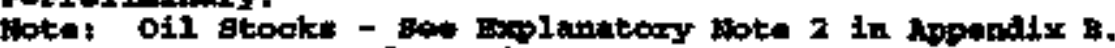

gotirces: toe and of geption 4 . 
Table 4.6 World oll Demand, 1970-1996

(rhoueand Earrela por Das)

\begin{tabular}{|c|c|c|c|c|c|c|c|c|c|c|c|c|c|c|}
\hline & & Prance & Itnly & 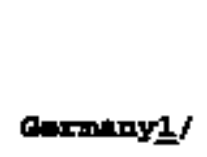 & $\begin{array}{l}\text { Onited } \\
\text { xinqdon }\end{array}$ & 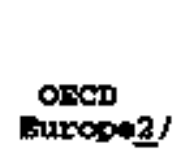 & $\begin{array}{l}\text { Onited } \\
\text { Btation } 3 /\end{array}$ & Canada & Japan & Other & $\begin{array}{l}\text { OxcD } \\
\text { actuding } \\
\text { East } \\
\text { Oerming }\end{array}$ & East & $\begin{array}{l}\text { Oexp } \\
\text { Including } \\
\text { Eact } \\
\text { Gorming }\end{array}$ & $\begin{array}{l}\text { Woxla } \\
\text { notal }\end{array}$ \\
\hline $\begin{array}{l}1970 \\
1971 \\
1972 \\
1973 \\
1974 \\
1975 \\
1976 \\
1977 \\
1978 \\
1979 \\
1990 \\
1981 \\
1982 \\
1983 \\
1984 \\
1985 \\
1996 \\
1997 \\
1998 \\
1999 \\
1990 \\
1991 \\
1992 \\
1993 \\
1994 \\
1995 \\
1996\end{array}$ & 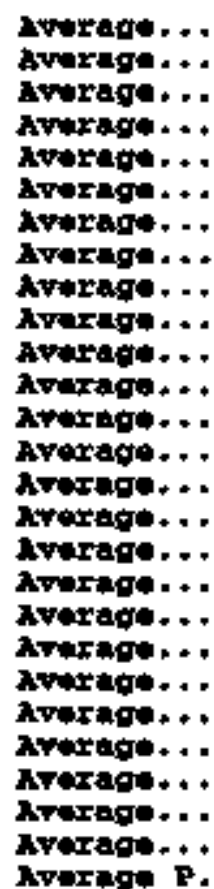 & $\begin{array}{l}1,937 \\
2,115 \\
2,322 \\
2,601 \\
2,447 \\
2,252 \\
2,420 \\
2,294 \\
2,408 \\
2,463 \\
2,256 \\
2,023 \\
1,860 \\
1,835 \\
1,754 \\
1,775 \\
1,772 \\
1,769 \\
1,797 \\
1,857 \\
1,819 \\
1,939 \\
1,926 \\
1,875 \\
1,833 \\
1,896 \\
1,935\end{array}$ & $\begin{array}{l}1,710 \\
1,838 \\
1,947 \\
2,068 \\
2,004 \\
1,855 \\
1,971 \\
1,897 \\
1,952 \\
2,039 \\
1,934 \\
1,974 \\
1,781 \\
1,750 \\
1,646 \\
1,717 \\
1,738 \\
1,655 \\
1,836 \\
1,930 \\
1,672 \\
1,863 \\
1,937 \\
1,852 \\
1,041 \\
2,060 \\
2,058\end{array}$ & $\begin{array}{l}2,607 \\
2,702 \\
2,859 \\
3,055 \\
2,740 \\
2,650 \\
2,877 \\
2,865 \\
3,927 \\
3,003 \\
2,707 \\
2,119 \\
2,372 \\
2,324 \\
2,322 \\
2,339 \\
2,490 \\
2,424 \\
2,422 \\
2,280 \\
2,382 \\
2,820 \\
2,843 \\
2,900 \\
2,879 \\
2,875 \\
2,911\end{array}$ & $\begin{array}{l}2,096 \\
2,141 \\
2,284 \\
2,341 \\
2,210 \\
1,911 \\
1,892 \\
1,905 \\
1,936 \\
1,971 \\
1,725 \\
1,590 \\
1,590 \\
1,531 \\
1,849 \\
1,634 \\
1,649 \\
1,603 \\
1,697 \\
1,739 \\
1,752 \\
1,801 \\
1,803 \\
1,815 \\
1,837 \\
1,845 \\
1,845\end{array}$ & $\begin{array}{l}12,004 \\
13,005 \\
13,934 \\
14,925 \\
13,998 \\
13,217 \\
14,124 \\
13,916 \\
14,290 \\
14,657 \\
13,634 \\
12,515 \\
12,053 \\
11,765 \\
11,736 \\
11,661 \\
12,102 \\
12,255 \\
12,427 \\
12,532 \\
12,629 \\
13,391 \\
13,605 \\
13,523 \\
13,597 \\
14,120 \\
14,269\end{array}$ & $\begin{array}{l}14,697 \\
15,213 \\
16,367 \\
17,308 \\
16,653 \\
16,322 \\
17,161 \\
18,431 \\
18,847 \\
18,513 \\
27,056 \\
16,058 \\
15,296 \\
15,231 \\
15,726 \\
15,726 \\
16,281 \\
16,665 \\
17,283 \\
17,325 \\
16,988 \\
16,714 \\
17,039 \\
17,237 \\
17,719 \\
17,725 \\
18,309\end{array}$ & $\begin{array}{l}1,516 \\
1,565 \\
1,664 \\
1,729 \\
1,779 \\
1,779 \\
1,810 \\
1,850 \\
1,902 \\
1,971 \\
1,873 \\
1,768 \\
1,578 \\
1,448 \\
1,472 \\
1,504 \\
1,506 \\
1,548 \\
1,693 \\
1,733 \\
1,690 \\
1,622 \\
1,643 \\
1,668 \\
1,727 \\
1,755 \\
1,797\end{array}$ & $\begin{array}{l}3,817 \\
4,142 \\
4,363 \\
4,949 \\
4,864 \\
4,621 \\
4,837 \\
4,880 \\
4,945 \\
5,050 \\
4,960 \\
4,648 \\
4,582 \\
4,995 \\
4,576 \\
4,384 \\
4,439 \\
4,484 \\
4,752 \\
4 ; 983 \\
5,110 \\
5,264 \\
5,446 \\
5,401 \\
5,674 \\
5,711 \\
5,867\end{array}$ & $\begin{array}{r}836 \\
831 \\
949 \\
998 \\
1,095 \\
1,041 \\
1,119 \\
1,160 \\
1,204 \\
1,178 \\
1,072 \\
1,080 \\
1,008 \\
954 \\
989 \\
976 \\
951 \\
959 \\
939 \\
998 \\
1,027 \\
1,056 \\
1,041 \\
1,218 \\
1,174 \\
1,227 \\
1,124\end{array}$ & 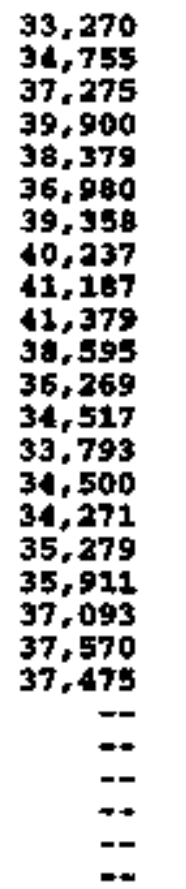 & $\begin{array}{l}223 \\
238 \\
270 \\
282 \\
307 \\
307 \\
329 \\
347 \\
363 \\
369 \\
375 \\
355 \\
371 \\
336 \\
340 \\
362 \\
362 \\
342 \\
322 \\
301 \\
293 \\
=- \\
-\square \\
-= \\
=- \\
-=\end{array}$ & $\begin{array}{l}33,493 \\
34,993 \\
37,545 \\
40,182 \\
38,686 \\
37,287 \\
39,687 \\
40,584 \\
41,550 \\
41,748 \\
39,970 \\
36,624 \\
34,898 \\
34,129 \\
34,840 \\
34,693 \\
35,641 \\
36,253 \\
37,415 \\
37,871 \\
37,758 \\
38,067 \\
38,768 \\
38,967 \\
39,890 \\
40,537 \\
61,366\end{array}$ & $\begin{array}{l}46,808 \\
19,416 \\
53,094 \\
57,237 \\
56,677 \\
56,198 \\
59,673 \\
61,026 \\
64,158 \\
65,220 \\
63,067 \\
50,903 \\
59,503 \\
59,744 \\
59,836 \\
60,091 \\
61,759 \\
62,999 \\
64,819 \\
65,917 \\
65,995 \\
66,577 \\
66,772 \\
57,043 \\
68,313 \\
70,193 \\
71,694\end{array}$ \\
\hline
\end{tabular}

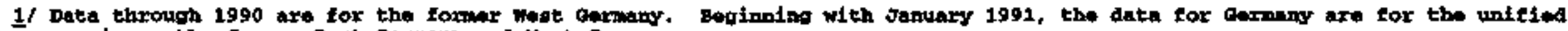

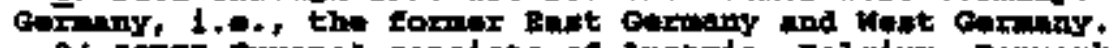

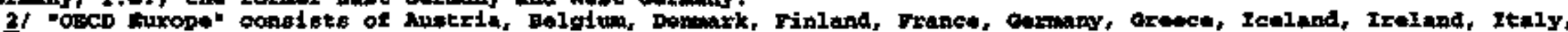

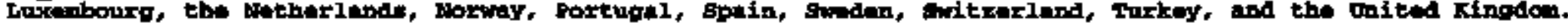

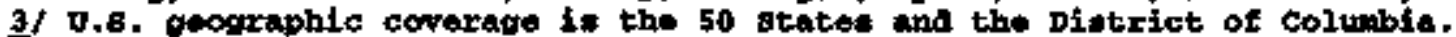

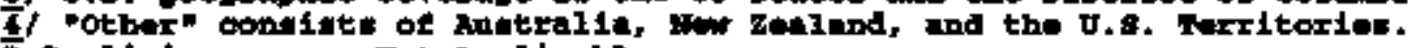

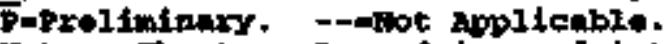

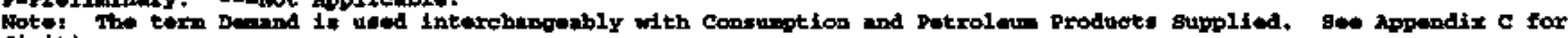

Gorinition.

Dourcest ses und of suetica 4 . 
Table 4.7 OBCD Total Ret Impozts, 1996-1996

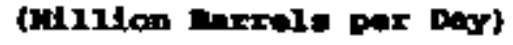

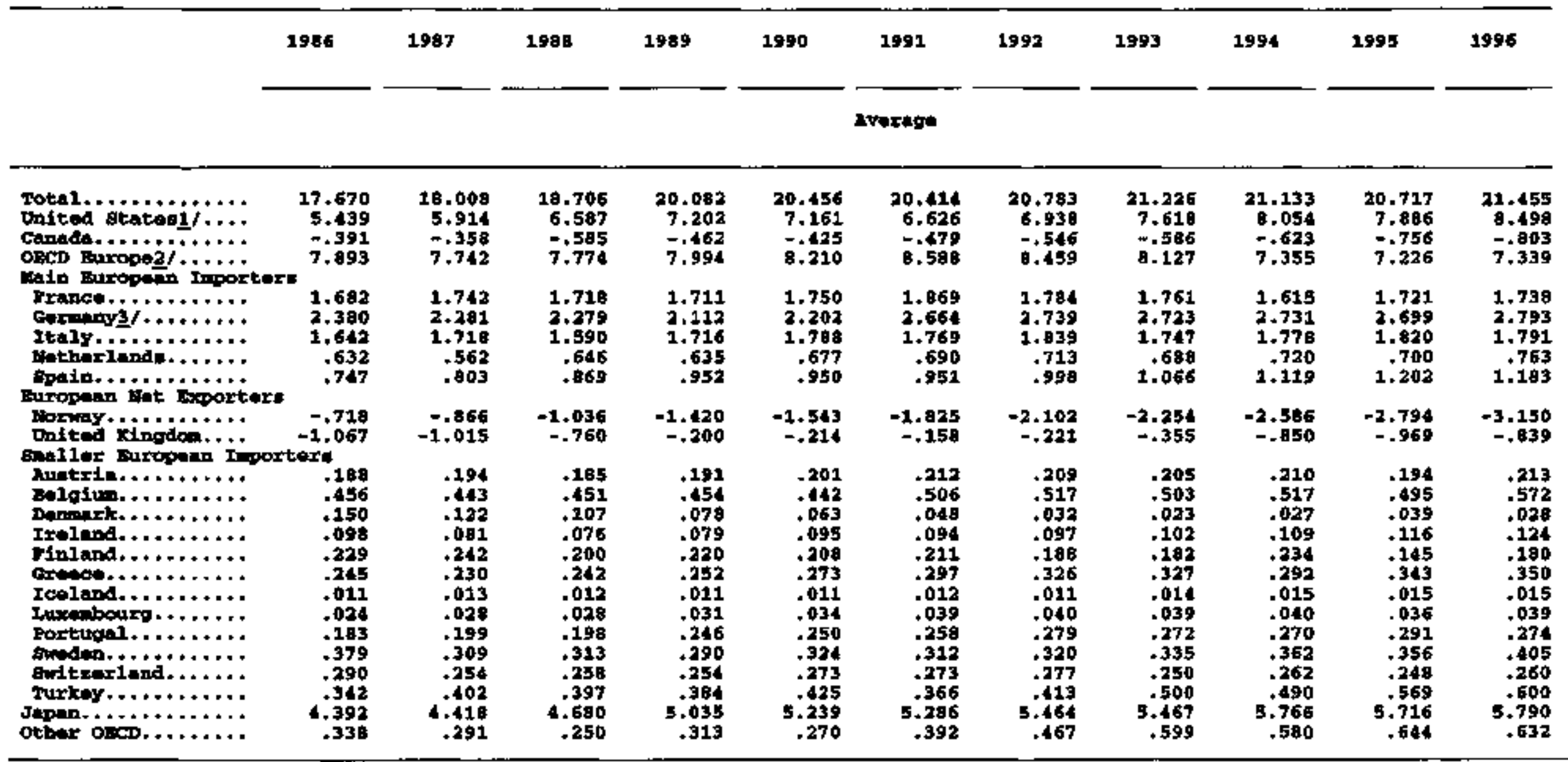

1) 0.8. Geographle cownase is the 50 stater and pistrict of coluble.

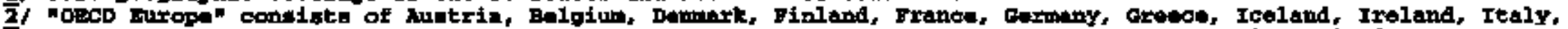

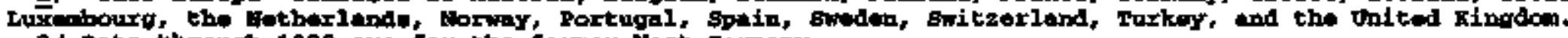

$3 /$ bate through 1990 aro tor the former fint cormany.

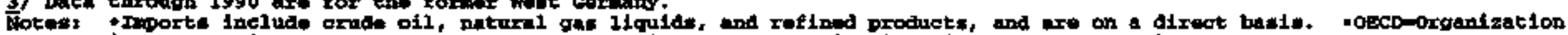

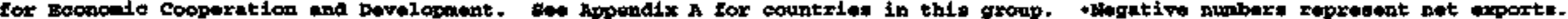

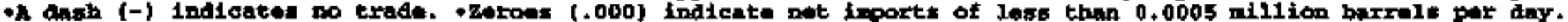
-Diti for 1995 ure preltminary.

bonrces: Bet ind of action 4 . 


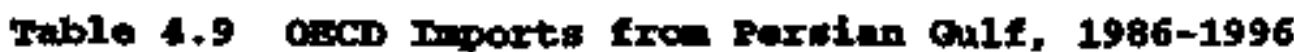

(riilion Barrele par Day)

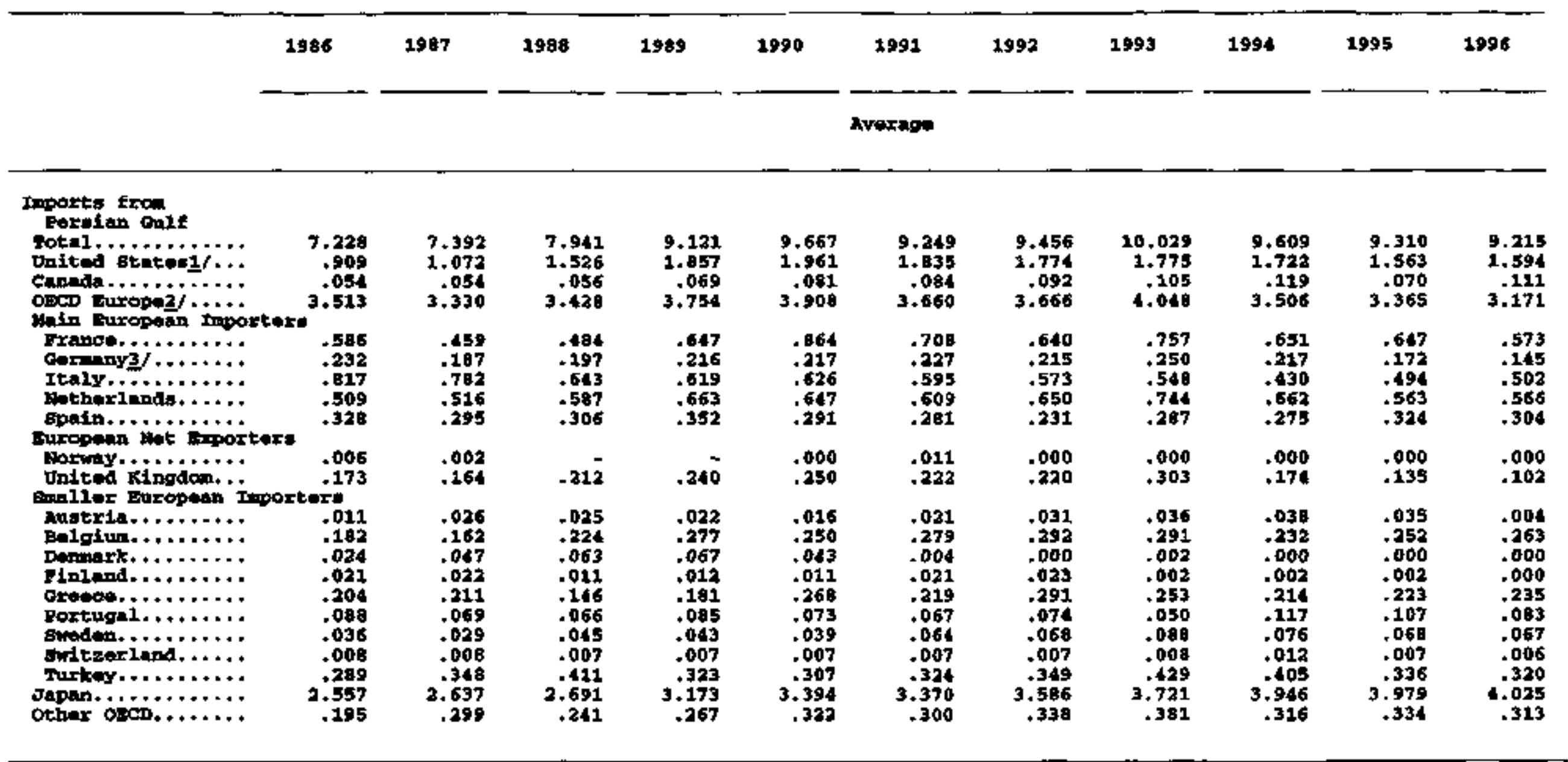

1/ U.8. Teographic coverage $1 \mathrm{~F}$ the 50 gtated and Diftriot of colimbla.

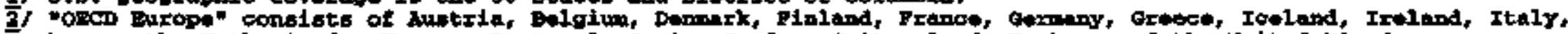

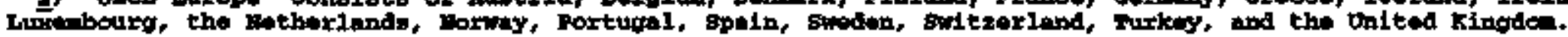

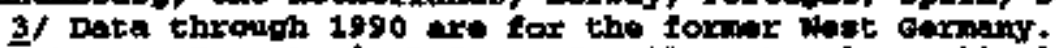

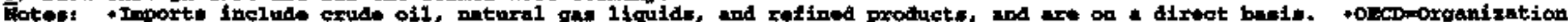

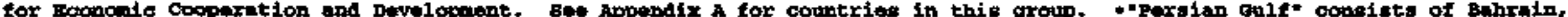

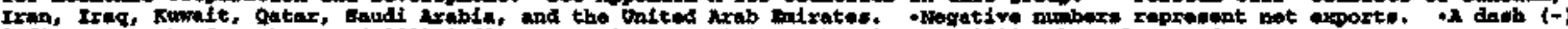

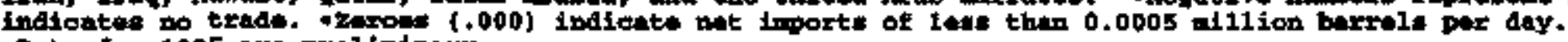

baten tor 1995 axe paplintmaxy.

Bouroed gie and of Bection 4. 
Table 4.11 Jagan--not ofl Inporta, 1986-1996

(aililon Burseld per Day)

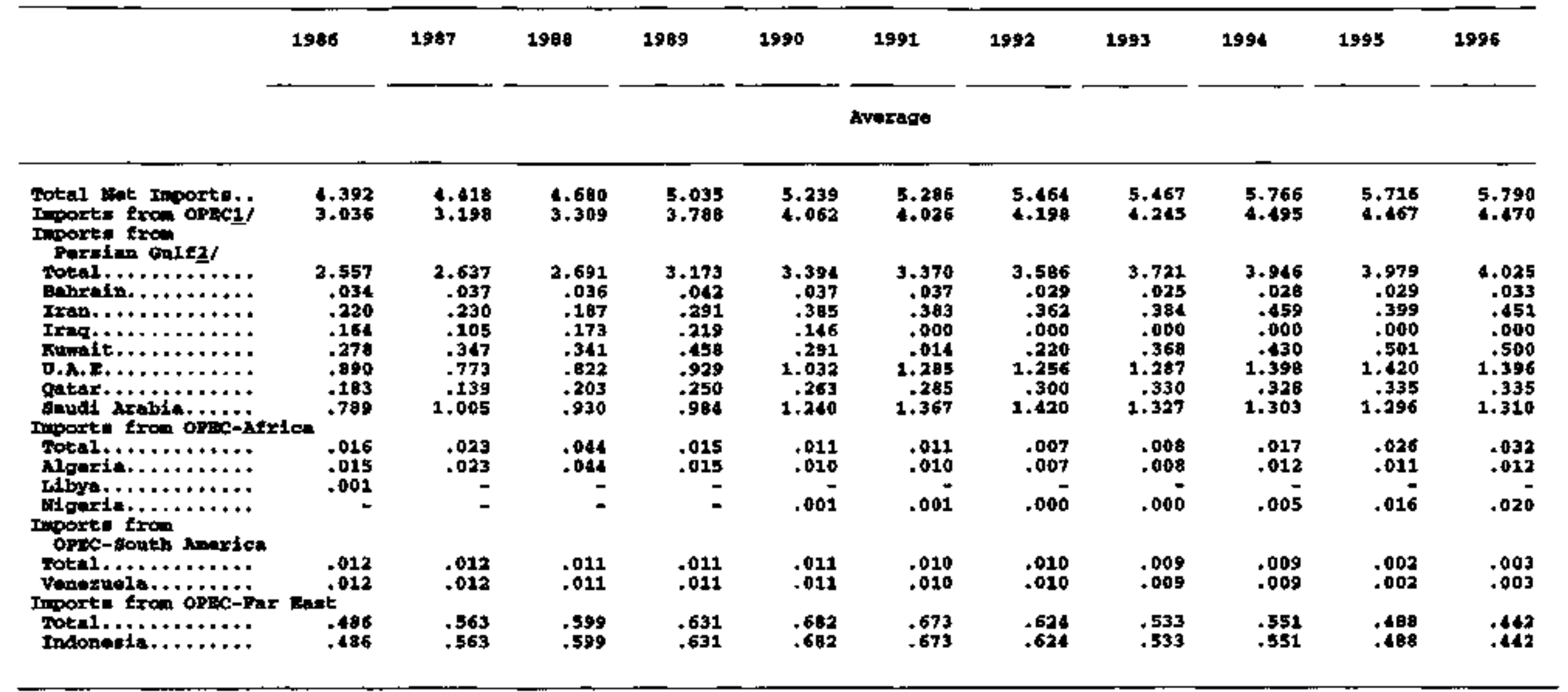

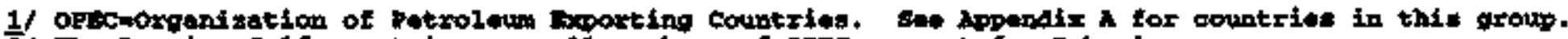

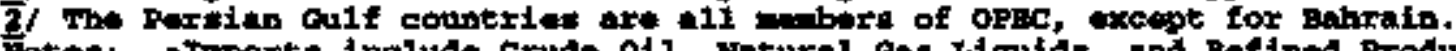

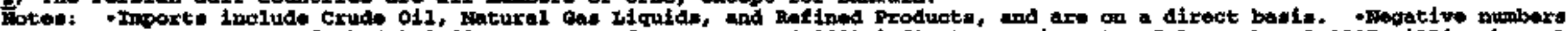

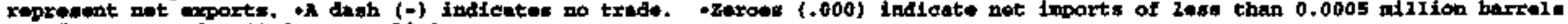
per day, -Date for 1995 axe preliminary.

Bourcant ate and of cection 4 . 


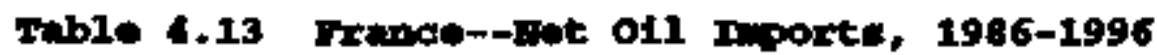

(Millias surmele par Day)

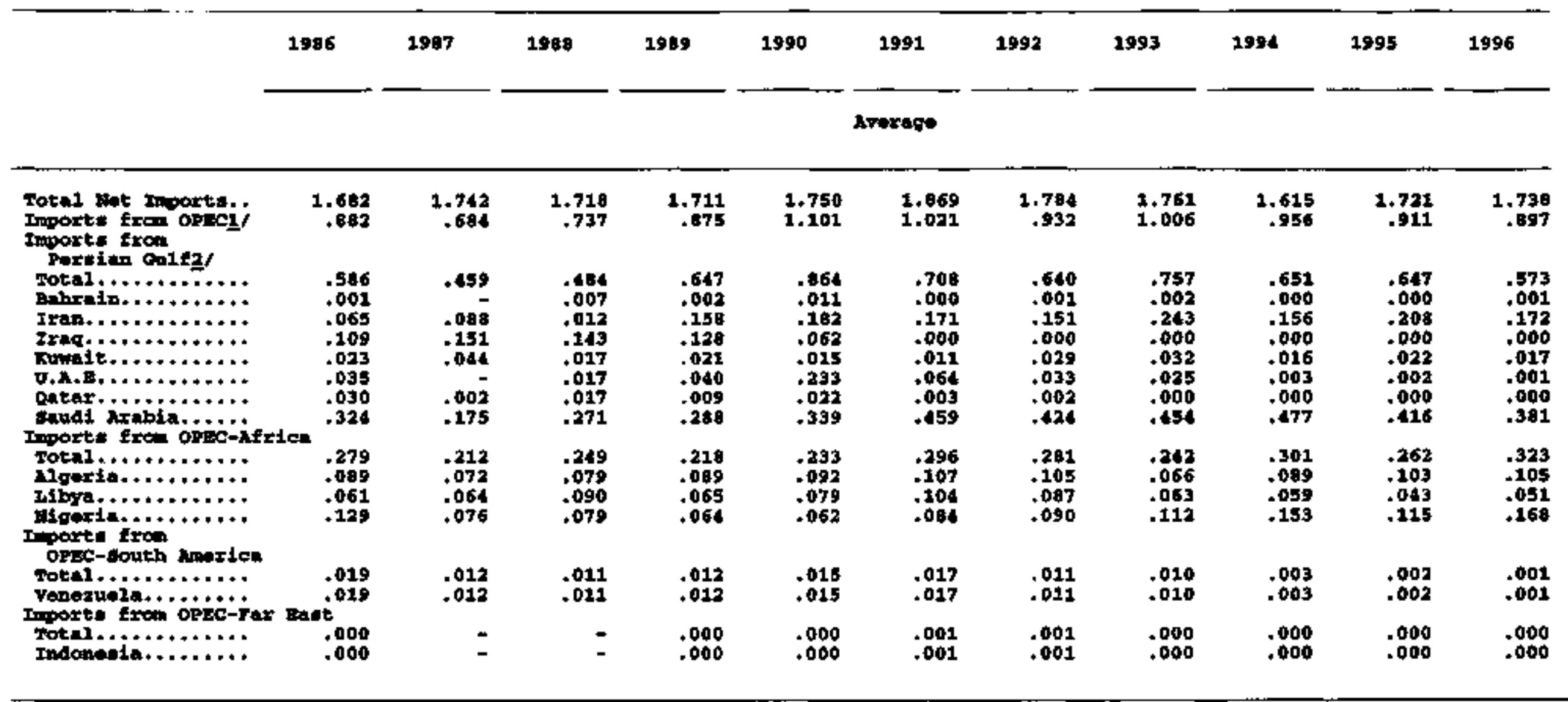

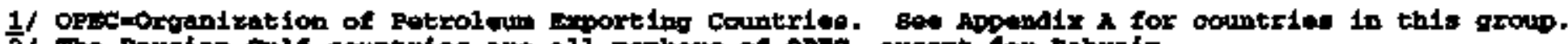

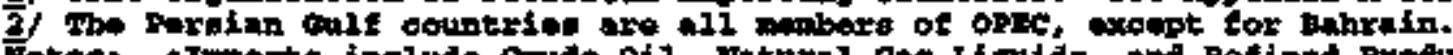

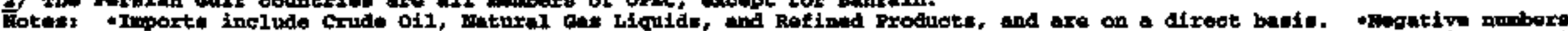

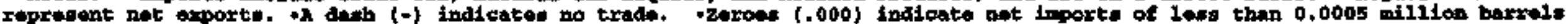
per day. Datn for 1995 axe prielintmiry.

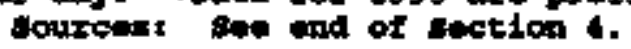


Table 4.15 Italy--Wet oil Imoxti, 1986-1996

(M11110n Burcoli: Der Daty)

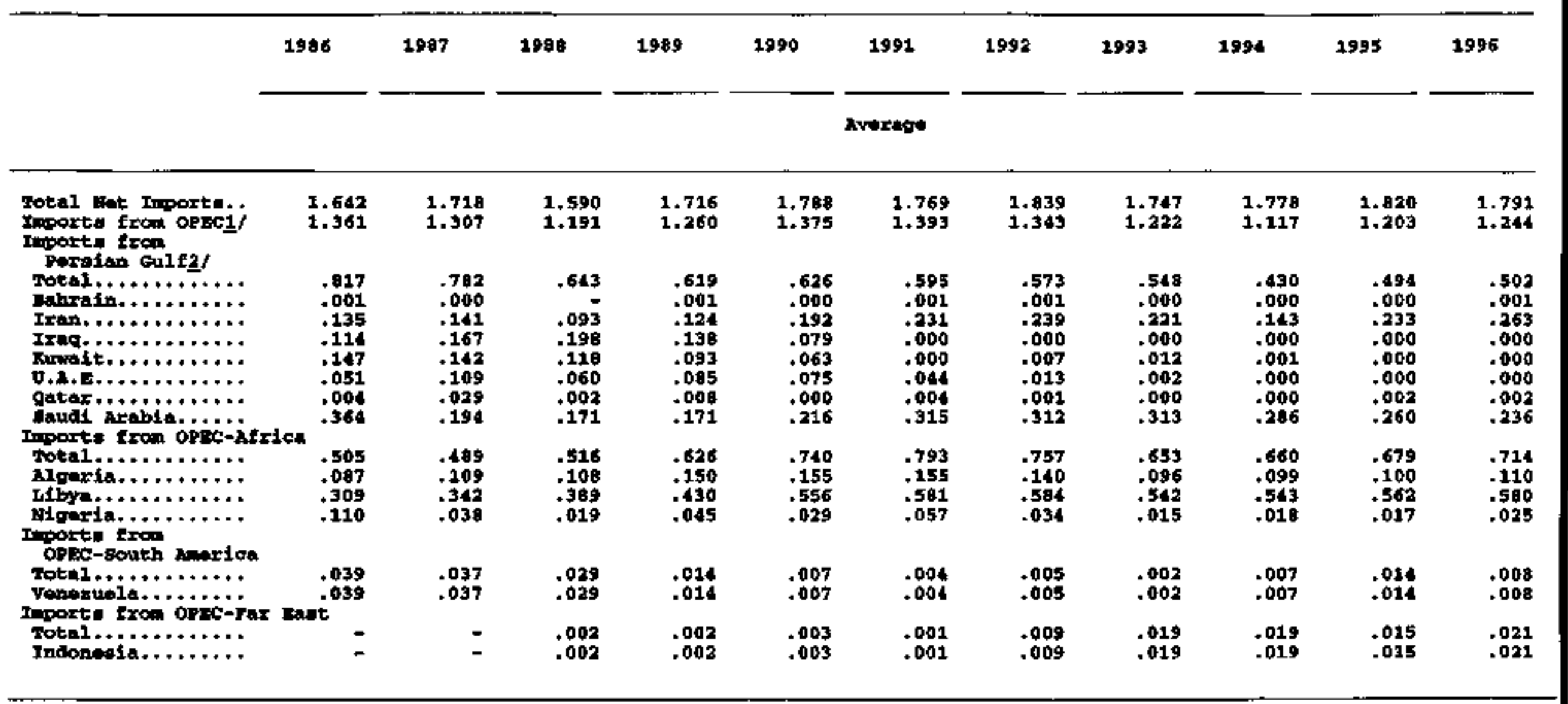

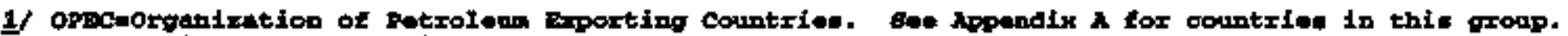

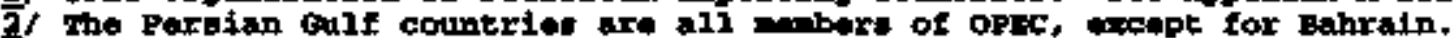

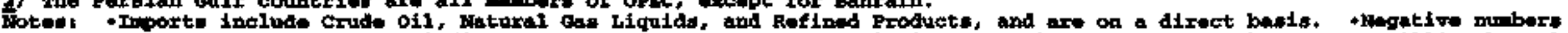

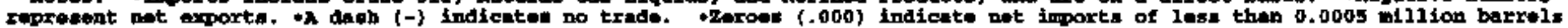
por asy. - Data for 1995 nre prelinimary.

scurded, Bet and of geotion 4 . 
Table 4.16 OxCD Iporte from Ireq, 1986-1996

(itillion Barroll por Day)

\begin{tabular}{|c|c|c|c|c|c|c|c|c|c|c|}
\hline 1906 & 1987 & 1988 & 1989 & 1990 & 1991 & $199 \lambda$ & 1993 & 1994 & 1995 & 1996 \\
\hline
\end{tabular}

\begin{tabular}{|c|c|c|c|c|c|c|c|c|c|c|c|}
\hline 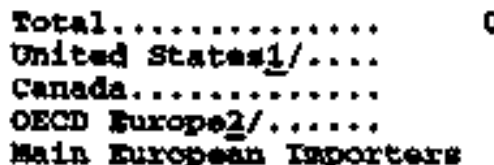 & $\begin{array}{r}0.964 \\
.081 \\
.7+7\end{array}$ & $\begin{array}{r}1.103 \\
.083 \\
.010 \\
.905\end{array}$ & $\begin{array}{r}1.489 \\
.343 \\
.001 \\
.959\end{array}$ & $\begin{array}{r}1.581 \\
.448 \\
.013 \\
.897\end{array}$ & $\begin{array}{r}1.276 \\
.511 \\
.011 \\
.591\end{array}$ & $\begin{array}{r}0.000 \\
.000 \\
.000 \\
.000\end{array}$ & $\begin{array}{r}0.000 \\
.000 \\
.000 \\
.000\end{array}$ & $\begin{array}{r}0.001 \\
.000 \\
.000 \\
.001\end{array}$ & $\begin{array}{r}0.000 \\
.004 \\
.000 \\
.000\end{array}$ & $\begin{array}{r}0.000 \\
.000 \\
.000 \\
.000\end{array}$ & $\begin{array}{r}0.007 \\
.001 \\
.000 \\
.006\end{array}$ \\
\hline 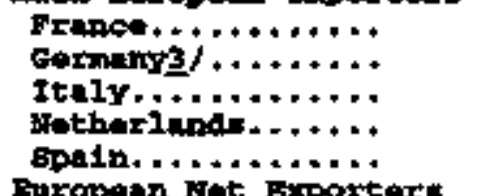 & $\begin{array}{r}.109 \\
.016 \\
.114 \\
.067 \\
.117\end{array}$ & $\begin{array}{r}.151 \\
.052 \\
.167 \\
.034 \\
.080\end{array}$ & $\begin{array}{r}.143 \\
.032 \\
.198 \\
.039 \\
.092\end{array}$ & $\begin{array}{l}.128 \\
.009 \\
.138 \\
.101 \\
.109\end{array}$ & $\begin{array}{l}.062 \\
.004 \\
.079 \\
.117 \\
.063\end{array}$ & $\begin{array}{l}.000 \\
.000 \\
.000 \\
.000 \\
.000\end{array}$ & $\begin{array}{l}.000 \\
.000 \\
.000 \\
.000 \\
.000\end{array}$ & $\begin{array}{l}.009 \\
.000 \\
.000 \\
.000 \\
.000\end{array}$ & $\begin{array}{l}.000 \\
.000 \\
.000 \\
.000 \\
.000\end{array}$ & $\begin{array}{l}.000 \\
.000 \\
.000 \\
.000 \\
.000\end{array}$ & $\begin{array}{l}.000 \\
.000 \\
.000 \\
.000 \\
.002\end{array}$ \\
\hline 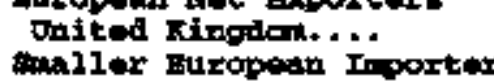 & .071 & .082 & .070 & .034 & .027 & .000 & .000 & .000 & . 4000 & .000 & .000 \\
\hline 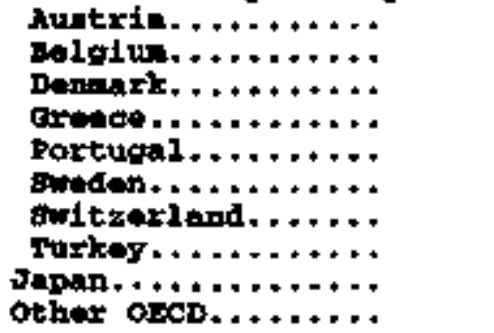 & $\begin{array}{r}.02 \overline{6} \\
-030 \\
.024 \\
= \\
.14 \overline{3} \\
.164 \\
.002\end{array}$ & $\begin{array}{l}.015 \\
.049 \\
.001 \\
.085 \\
.019 \\
.003 \\
.000 \\
.108 \\
.105 \\
.000\end{array}$ & $\begin{array}{r}.007 \\
.037 \\
-059 \\
.029 \\
.003 \\
. \\
.250 \\
.173 \\
.023\end{array}$ & $\begin{array}{r}.000 \\
.047 \\
.052 \\
.024 \\
.005 \\
.000 \\
.250 \\
.219 \\
.003\end{array}$ & $\begin{array}{l}.000 \\
.029 \\
.000 \\
.041 \\
.019 \\
.000 \\
.000 \\
.146 \\
.116 \\
.011\end{array}$ & $\begin{array}{l}.000 \\
.000 \\
.000 \\
.000 \\
.000 \\
.000 \\
.000 \\
.000 \\
.000 \\
.000\end{array}$ & $\begin{array}{l}.000 \\
.000 \\
.000 \\
.000 \\
.000 \\
.000 \\
.000 \\
.000 \\
.000 \\
.000\end{array}$ & $\begin{array}{l}.001 \\
.000 \\
.000 \\
.000 \\
.000 \\
.000 \\
.000 \\
.000 \\
.000 \\
.000\end{array}$ & $\begin{array}{l}.000 \\
.000 \\
.000 \\
.000 \\
.000 \\
.000 \\
.000 \\
.000 \\
.000 \\
.000\end{array}$ & $\begin{array}{l}.000 \\
.000 \\
.000 \\
.000 \\
.000 \\
.000 \\
.000 \\
.000 \\
.000 \\
.000\end{array}$ & $\begin{array}{l}.000 \\
.000 \\
.000 \\
.000 \\
.000 \\
.000 \\
.000 \\
.003 \\
.000 \\
.000\end{array}$ \\
\hline
\end{tabular}

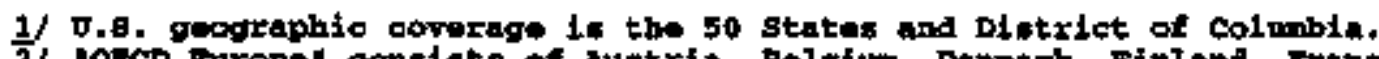

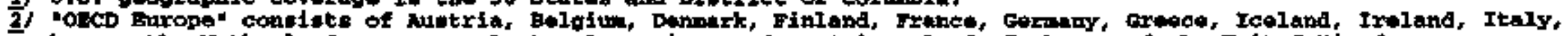

In

$3 /$ Date through 1990 ar for the formor Woit Germany.

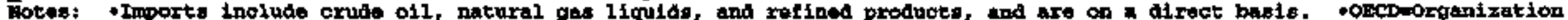

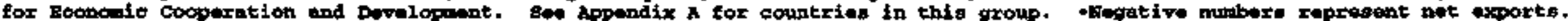

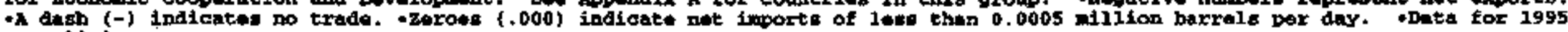

r. prolininary.

Bourcost Bine and of soction 4. 
Table 4.17 O.CD Importe Ercm Tuwat, 1986-1996

(Mililion Barrele pax Day)

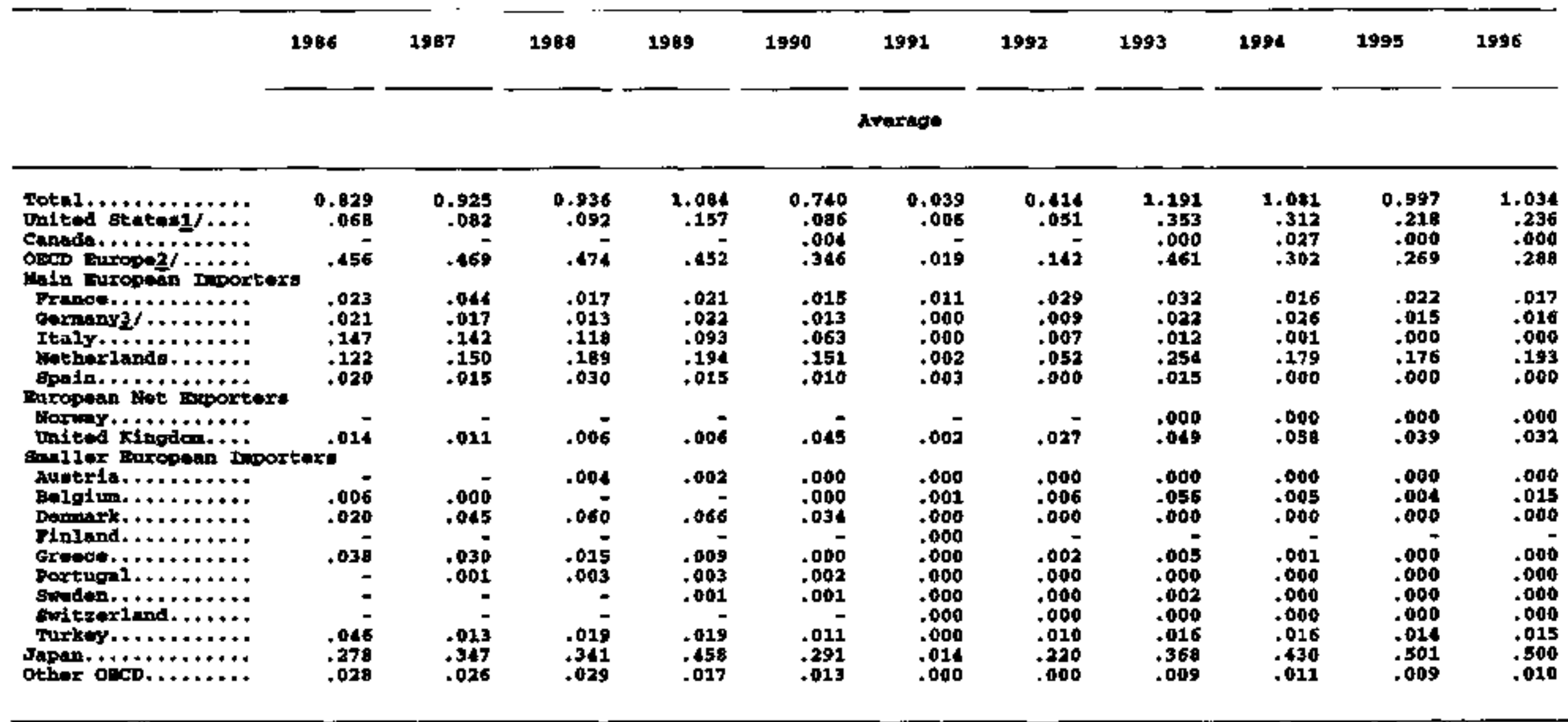

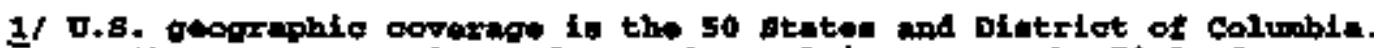

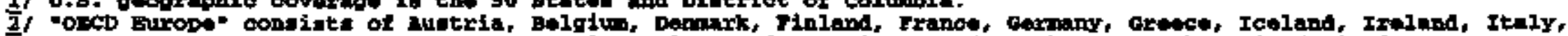

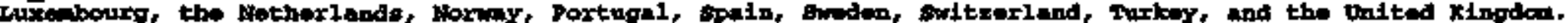

$3 /$ Deta throtob 1990 axe for the formor wist corcams.

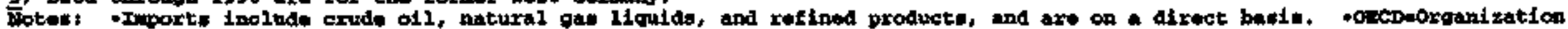

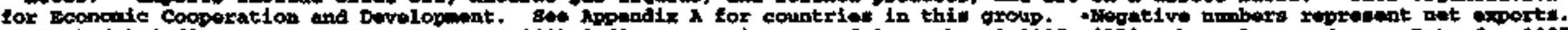

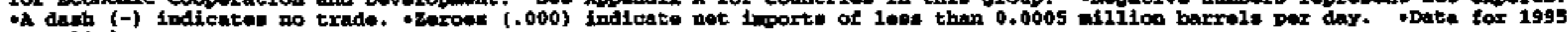
are prelininary.

Ecurged son and of section 4. 


\section{Sources for Section 4 (Historical Oil Data)}

Crude Oil Production, Natural Gas Liquids Production, Other Liquids, and Refinery Gain

United Ststes: • Energy Information Adninistration (EIA), Petroleum. Supply Anmual 1996; - Bunean of Mrzes, Mineral Industry Surveys, Petroleam Slatement, Annulal, various issues.

Other Countries: - ELA, Intemsational Egesgy Annual, various issues. - ELA, Irtemrationzd Petroleum Annual, 1978. - United Nations, Yearbosk of World Energy Staristics, 1979. Refinery Gains: National Petroleum Councit, U.S. Peinoleum Refinery Saudy, October 1986.

Oil Stocks

United States: BIA, Petroleum Supply Anmital $19 \%$.

Oher OECD Countrtes: * 1973-1982: Orgmization for Ecrmonic Cooperation and Devekpnenx (OECD), Quanterly Qil Staistics and Energy Balances, various issues. 1983-1997; OECD, Monthly Oil Strtistics in the ELA, Intemational Energy Datalase.

Oil Dentarid

Unised Stetes: ElA, Petroleum Sueply Anmeal 1996.

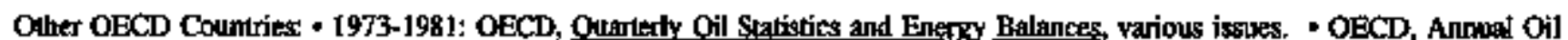
Statistics data base, 1970-1979. - I982-1997: OECD, Monthily Oil Statistics in the FIA Intemationsl Energy Database.

Oil Imports

United States; Energy Information Administration (EIA), Petroleum Supply Annual 1996.

Other OECD Countries: - Organization for Eecntomic Cooperation and Development (OECD), Cuarterly Oil Statistics and Energy Balances various issues. - OECD Monthly Oil Stakstics datahase, 1991-1997. 

Appendix A

Organizational and Geographical Definitions 


\section{Appendix A. Organizational and Geographical Definitions}

International Enerzy Agency (EEA) countries are: Ausıralia, Austria, Belgiom, Canada, Denmark, Firlend, France, Gernany, Oceece, Ireland, Jtaly, Japan, Luxembourg. Netherkands, New Zeakand, Norway, Portugal, Spain, Sweden, Switzerland, Turkey, United Kingdon, and United Slales.

Orpanization for Foonomic Cooperation and Development (OECD) coumries ire: Australia, Austria, Belgium, Canada, Czecth Republic, Denmark. Finland, France, Germany, Greece, Hungary, Iceland, Ireland, Italy, Japan, Luxembourg, Mexico, Netherkands, New Zealand, Norway, Poland, Pontugal, South Kurea, Spain, Sweden, Switzerland, Turkey, United Kingoom, and United Stales. Total OECD includes the Uniled States Teritories (Guam, Puerto Rico, and the U.S. Virgin Islands), Total OECD excludes dala for Czech Republic, Hongary. Mexico, Poland, and South Korea which are not yet availabte.

OECD Eurepe countries are: Austria, Belgium, Czech Republic, Denmark, Fimland, France, Genmany, Grece, Hungary, Iceland, lreland, haly, Luxembourg, Netherlands, Norway, Poland, Portugai, Spain, Sweden, Switzerland, Turkey, and Utriled Kingdom. OECD Europe excludes daka for Czech Republic, Hungary, and Poland which ane not yet avaiłable.

Organimation of Petroleam Exportiag Countries (OPEC) menters ae: Algeria, Intonesia, Iran, Iraq, Kuwait, Libya, Nigeria, Qatar, Saudi Arabra, the United Arab Enirates, and Veneavela. As of Decenber 31, 1992, Ecuador withdrew its membership in OPEC, and as of December 31, 1994, Gabon withdrew its membership. For consistency. Ecuador and Gabon ane excluded in the historical series of OPEC totals.

Arab inemabers of OFEC are: Algeria, Iraq, Kuwail, Libya, Qatar, Saudi Arabja and the Unitcd Arab Emirates.

Organization of Arab Petroleum Exporting Countrites (OAPEC) are: Algeria, Bahrain, Egyjx, Iraq, Kuwait, Libya, Nenxral Zone, Qatar, Saudi Arabja, Syria, Tunisia, and the United Arab Emizales. In April 1979 Egypt was suspended from OAPEC membership. In May 1989 Egypt rejoined OAPEC. For consistency. Egypt is inchuded in the historical series of the DAPEC totals.

Persian (Arabian) Gulf countries are: Bahrain, Iran, Iraq, Kuwgit, Qatar, Saudi Arabia, and the United Arab Emirates.

The term "Former USSR" is being used in the Inlimational Peitulenm Sististics Report as a purcly geographical expression, referting to the Commonwealth of Indeperident States (CLS), Georgia, and the three Baltie States (Estonia, Latvia, and Lillwania). 


\section{Appendlx B}

\section{Explanatory Notes}




\section{Appendix B. Explanatory Notes}

Note 1: World Oil Supply

Supply inclvdes production of crude oil (inclucing lease condensale), nalural gas plant liquids, other hydrogen and hydrocartons for refinery feedswocks, refinery gain,

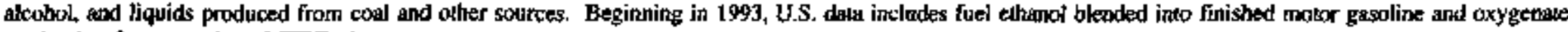
production from merchant MTBE planls.

\section{Note 2: Oil Stocks}

The International Energy Agency (EA) assembles and reports totat oil stock data for the member couttries of the Organization for Economic Cooperation and Development (OECD). Oil stueks include crude oil (itcluding stralegic reserves), unfinistred oils, natural gas plimt liquivis, and refined peiroleum prodkcts. In its report titled Ouarterly Oil Stalistics and Energy Balances of OECD Coumlites, the IEA defines oil stocks as follows: "All non-military stocks held by importers (iticluding all final consumers directly?, refiners, Govermments, majos non-importing final consumers eligite under IEAFOECD commitments and by foreign entities in the following facilities: bulk tenminals, refinery knks, pipeline tankage, barges, intercosstal tankers (when pon of departuse and destination are in the reporting country), tankers in porl (to the extem that their cargo is to be unloaded in the reporting country), and inlond ship bunkers. Oil is reported regandless of the ownership of such storsge facilities." IEA data exclude "oil held in pipelines, sail and truck tank cass, sea-going ships' bunkers, service stationts, relail slores and tankers at sea." Also the IEA defines the United States to be the 50 States and the District of Cobmbia, and the US. Territories (Gaam, Puerto Rico, and the U.S. Virgin Islands). The Energy Information Administration (ElA) does not include the United States Territonies in its statistical definilion of the United States. Further, the EIA includes oil in oil pipelines as part of its stock duta. Tables 1.5, 1.6, 2.3, and 4.4 include oil stocks for the United Slates according to EIA definitions. Using IEA definitions, at tibe end of Sepuember 1997 United Stales (inchuding terrilories) oil stocks totaled 1,450 million barrek.

Note 3: A Change to the Oil Balance in Section 2.

Prior to August 1992, Section 2 of the Internatignal Petooleum Stmistics Report presented a balance of oil supply, denand, and slock chinge for the Market Economies. at quarterly time intervals. The commutuisl countries, or Centrally Planned Ecchomies (CPEs) were not ineluded in the balance, mainly because good anarterly data for oil demarnd in these countries were not ayajlable. Net imports fiom the CPEs were included in the balance as part of toal Market Economies supply. Al presenn, the former Sovict Union and most of the countries of Eastem Europe are in transition from communisen to market econonies; because of this there are no longer good political or economic reasons to publish an oil balance only fox the countries that have traditionally been market economies. Beginning with the August 1992 edition. Section 2 of the Intemational Petrokum Statistics Report was changed from a Matket Economies oil balance to a world oil balance.

The main dala issues related to this change may be summarized as follows:

Supply Dala Monthly oil supply daka are available for all coumuries of the works These data are used to generate Tables 1.1 to 1.4 ; they were used to expand the Section 2 supply lable (Table 2.2) to the whole world.

Demend Datag. ELA has now developed quarterly figures for word oil demand, inchuding demand in the fornyer CPEs; these are published in Table 4 of ElA's Shont. Tem Energy outlook. The procedures involved im developing quartetly world demand mumbers may be summarized as follows: 
Complete rnorathy data are availabte for the 24 OECD courtries. Annual demand dala for all countries (about 200 counaries) are presented in ELA $s$ Intermational Energy Annual. In 1993, the OECD accounted for ahout three-fifths of total world demand, the 25 largest consumers ousside of the OECD accounted for about onethith of the world total, and the remaining countries (aboet 150 comtries) accounted for less than onte-tenth of the tocal. The key probtern, therefore, is to develop gond figures for the 25 largest consumers outside the OECD.

Of these 25 countries, by far the largest consumer is Russia, which in 1993 accoutncd for about one-sixth of the demand in the group of 25 . The next largest consumer is Chinz, which in 1993 accounted for about one-sewerah of the demind in the group. Thus in 1993 Russia and Chitha accounted for onc-lhird of the demand in the group of 25. For these 2 countries, data are available on oil production and net exports. The demand numbers shown in Section 2 are computed as production minus net exports. This is the stome procedure tsed in the EIA Short-Tenn Energy Outbook, by the Intemational Energy Agency in their Monthly Oil Manket.Report, and in other respected publications that deal wilt intermalional oil markets. Note, however, that this method of computing demand ignores bohh refinery gain and stock changes.

For other major consuming countries outside of the OECD, quarterly dentand data are developed based upon the latest available annual data, analytic judgrnent about currest trends, and some seasonal adjustments. These are the same procedures used for the Shont-Term Energy Outlook.

Slocks Dala. Reliable stocks data are available for the OECD countries. Estimates of stocks for export and afloat, and of comtnercial floaling stocks, are available. (See sources at the end of Section 2.)

For on-land stocks outside the OECD, there is litule reliable data. Most estimales of on-land stocks outside the OECD are based on the theory that stocks are approximately equal to some number of days of demand. Demand figures (which themselves involve some degree of estimation) are then used to compute estimates of stock levels.

In the Interrational Petroleum Statistics Repost, it had been the practice for some time to estinale that on-land stocks in the tractitional market economies outside of the $O E C D$ were equal to 55 days of demand. This was aceptable, mainly because the ruk was used only to cstimate a relatively small part of total stocks (about 16 percent).

When Section 2 was converted from a Market Exonomies oil balance to a world oil balance, there were important changes in the contilions that made it reasonable 10 estimate on-land stocks oulside of the OECD using the rule of 55 days of demand. When the CPEs ace inclukfed, the stocks that must be estimated are a considerably larger pant of the total. In 1990, total oil demand in the former CPEs was more than 80 percent as large as denand in all of the nom-0ECD traditional market economies. Thus the quantity of stocks that would have to be estimated using a 55-tay nuk would newrly double, and the estimated components of siock levels and stock changes would become relatively large parts of the total. One consequence of this is that the statistical discrepancy (as shown in Table 2.1) woukd become less usefol as an indicator of the overall consistency of the data. The statistical discrepancy is computed from supply, demanid, and slock change. IS a large part of the stock change is estimated, then the statistital discrepancy loses much of its significance.

As already noled, quarterty demands in tłe former Soviet Union and in China are computed as production minus net exports. This computation implicitly assumes a zero slock change. Demand in the former Soviet Union is falling. If we were to use a 55-day rule to compute stock levets, falling demand would imply falling stock levels. This would contradict the assumption of zero stock change which was used in compuling demand. 
In view of all this, the following approwh has been adopted. Table 2.3 now shows bveis of stocks for the OECD countries, levels of stocks for expont and affoat, and levels of commercial floaling slorage. Slock changes based on these stock kvels are shown in Table 2.1. At this time, no okher stock kevels are showi. Tahle 2.1 now has a single line labelled "Other Stock Draw and Statistical Discrepancy", which contains the computed values meeded to make the table balance.

Il may become possible in the future to obliain reliable informalion on certain categories of on-land stocks oulside the OECD. If such dala become ayailable, they will be added to Table 2.3 , and the cornesponding stock change information will be added to Takle 2.1 .

Changes.in Eomats and Sign Conventions. There wene several changes in Section 2 to make the Intemalional Fearoberm Statistics Repon mote consistent with ofher ElA publications, especially with the Shont-Tem Energy Outhook. The numbers were changed from I decimal place to 2 decimal places. The word "consumption", as used before August 1992, was changed to "demand". The sign conventiont for stock changes in Table 2.1 was changed. Siock draws are now indicated by positive numbers, and stoct additions are indiczted by negative aumbers. Table 2.1 also uses a new sign convention for the line labelled "Other Stock Draw and Statistical Discrepancy". The valuss in this line are now cemputed as Total Demand minus the sum of Total Supply and Total Repoted Stock Draw. This is the reverse of the sige convention used before August 1992.

Summary. Sextion 2 was expanded from a Market Economies oil balance to a world oil balance. Supply data are treked for all producting countries. Dernand data are tracked for the 24 OECD countries, and for 25 large consumers oukside of the OECD. Demand for the romaining cournries (less than 10 percent of total demand) is estimated, for years that are not yet available in the Intemational Energy Annual The stoct levels in these tables are based on reporled data for the OECD and for stocks at seal. The line in Table 2.1 labelted "Other Stock Draw and Statistical Discrepsincy" is a balancing ilem. 
Appendlx C

Giossary 


\section{Appendix C. Glossary}

Alcohol. The farily name of a groutp of orgatite chemical compoutads composed of carbon, hytrogen, and oxygen. The molecules it the series vary in chain length and are composoed of a bydrocarton plus a hydroxyl group. Atcohol inciudes methanol and ethanol.

Demend (Consomptton/Petroleum Products Supplied). Total petroleum products supplied is the sum of all petroleum products supplied. For each product, the amount supplied is calculated by summing production, cnude oil burned ditectly, imports, and net wilhdrawals from primary slocks and subtracting exports.

Crude 04. A mixture of hydrocarbons that existed in liquid phase in underground reservoirs and remains liquid at atmospheric pressure after passing turough surface-separating facilities. Crude oil production is measured at the wellheast and includes lease condensane.

Goverument-Owned Stocks. Oil stocks owned by the national govesnment and held for national secucily. In the Umited States these stocks are known as the Strategic Petroleum Reservo.

Lense Condensate. A liquid recovered from nisural gas at the well or at small gasjoil sepsiators in the field. Consists primarily of pentanes and heavier hydrocartons (also called field condensaxe).

Natural Gas Plant Liquids. Products obtained from processing nazural gas al nalural gas processing plants, including natural gasoline plants, cycling plants, and fractionators. Products ublained include ethane, liquefied pelrokeum gases, (propanes, bularre, propane-butants mixtures, and ethane-propane mixtures), isopentanc, natural gasoline, unfraclionstcd streams, plant condensere, and other minor quantities of finished products, such as motor gasolitte, special naphthas, jet fuel, kerosene, and distillate fuel oil.

Oil Stocks, Oil stocks include crude oil (including strategic reserves), unfinished oits, natural gas plaxk liquids, and refined pelroleum pauducls. See Explanatory Note 2

Other Hydrocarbons. Other maserials processed at refineries. Includes coal tar derivatives, hydrogen, gilsonite, and nalural gas received by the refinery for reforming imo hydrogen.

Oxygenates, Any substance which, when added to gasoline, increases the anount of vxygen in that gasoline blend. They include: Fuel Ethanol, Methanol, and MTBE (Methyl tertiary butyl cther).

Petroleum Products. Petroleum producls are obtained from the processing of crude oil (including tease condensate), natural gas, and other hydrocartion compourds. Petroleum products include unfinished cils, aviation gasoline, motor gasoline, naphtha-type jet fuel, kerosene-fype jel fuel. kerosene, distillale fuel oil, residual fuel oil, ethane, liquefied petroletun gases, pelrochemical feedstocks, special naphthas, lubricants, paraffin wax, petroleum coke, asphalt road oil, still gas, and other miscellaneous products.

Processing Gain. The amount by which the toull volume of refinery outpul is grealer than the volume of input for given period of time. The processing gain arises when crude vil and other hydrocarbons are processed into prochicts that are, on average, less dense than the input.

Processing Lass. The amount by which the total volume of refinery outpul is less thas the volume of input for given period of tient. The processing loss arises when crude oil and cather hydrocarbons are proccssed inlo products that are, on average, more dense than the ingut. 


\section{Appendlx D}

Estimation Procedures 


\section{Appendix D. Estimation Procedures}

Estimation procedures ane used for denand numbers in minly of the coumeries outside of the OECD, in cases where data from ElA's Internerional Enecgy Annual ane not yet available. Demand numbers for 1995 and eartier years, for all counnries, may be found in ELA's Intemational Energy Anmual 1995 , published in December 1996, which is the most recent published edition of the Intemational Energy Annual. EIA's WORLD ENERGY database conteins final demand data for all countries of the world for 1994, and preliminary demand data for alt countries for 1995 .

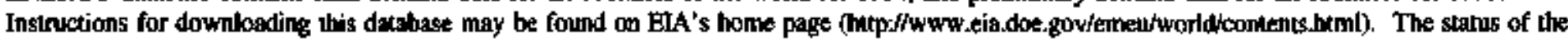
demand numbers shown in Section 2 of this cument Intemational Petroleum Statistiss Report may be summarized as follows.

The OECD demand numbers are, as usual, based on the latest Quertedy Oil Statistios and Monthly Oil Stalistics tapes trom the Intemational Energy Agoney.

For coumiries outside of the OECD, the following approach is used. The cumbers for 1994 and earlier years match the demand numbers in the WORLD ENERGY dasibase on EIA's home page. Numbers for 1995 forwand ane the best arribable EIA estirnates. based upon the best antusal dala currently available, analybic judgment about current tmends, and somx seasonal gdjusıments. These are the same numbers that appear in the international oil balance table in EjA's shon-Term Energy Outkok.

The 1wo largest non-OECD oil consumers are the former Soviet Union and Ching. For these two coumtries, publishad data are available on production and net exports. Denand is computed as production minus net exports. This is the same approach used in the Shart-Term Enersy Outhols, by the Intemational Energy Agency in their Monthly Oil Market Report, and in other major publicalions dealiag with international oil data. Nole, however, that because of this computation, the demand numbers for the former Sowiet Unirn and China implicitly exclude stock draw and refinery gain. 
Appendix E

Conversions 
Table E1. Refined Petroleum Products Conversion Factors

Product

Barrels per Metric Ton

Asphalt

Distillale Fued Oi

Gasoline, Avimion

Gasoline, Motor

Greases

Jet Foed, Kerosene-lyp

Jet Fued, Naptho-lype

Kerogene

Liquefied Peiroleum Gas (LPG

Lubricants

Miscellareous Produets

Naphthas

Nanural Gas Liquids (NGL)

Nanural Gasoline

Proratin

Paraftin Wax

Peirolahum

Petroleum Coke

Residual Fuet Oil

White Spirits 
rable E. Berrels of Crude ofl por Latric Ton, 1986-1995

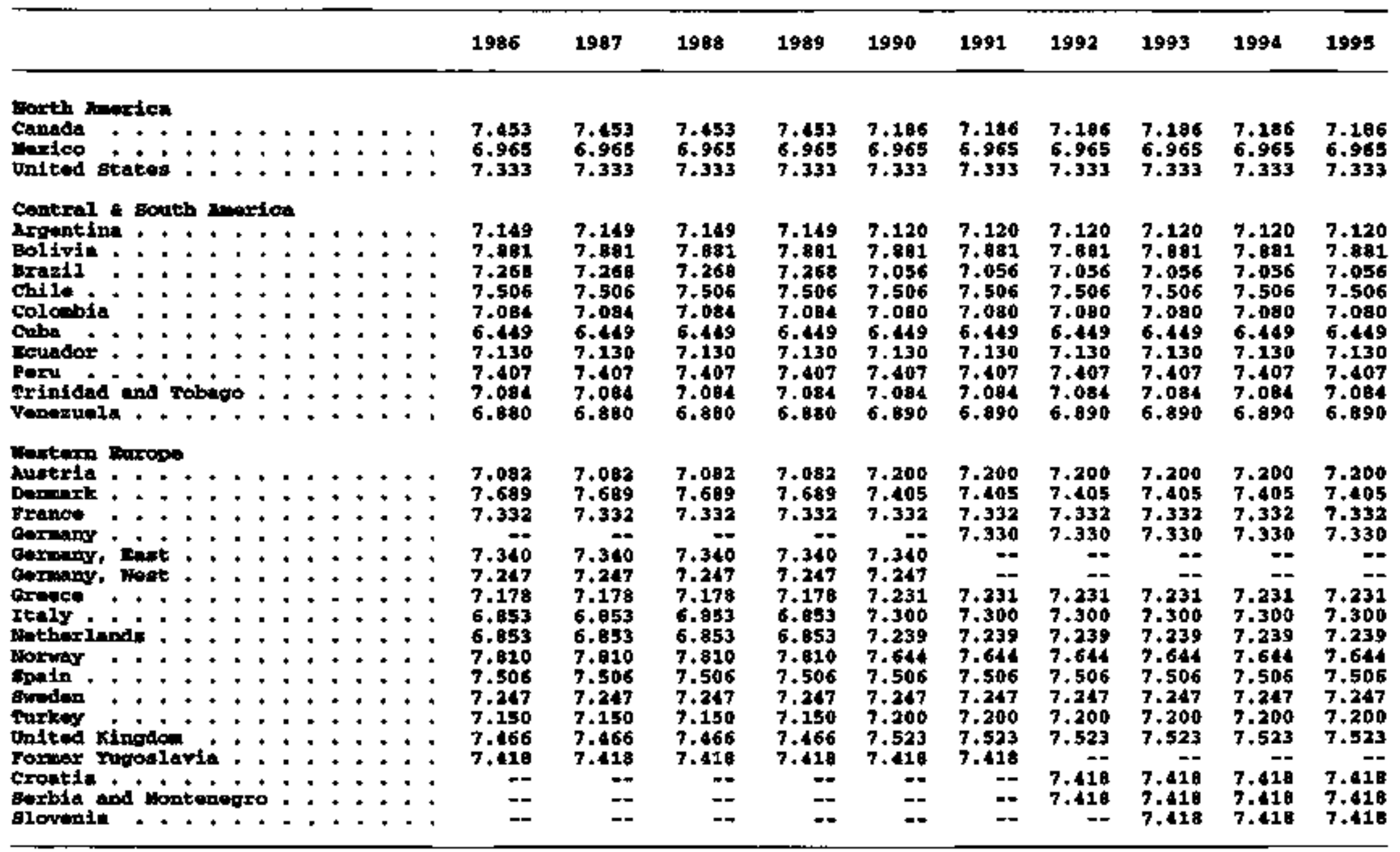

-- a bo production of tbil fort of enory

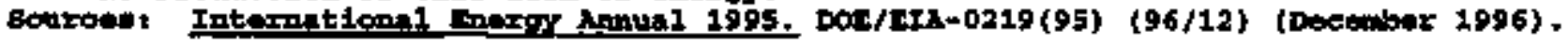


Fable 72. Barrels of Crode ofl par Hetric non, 1986-1995 (continued)

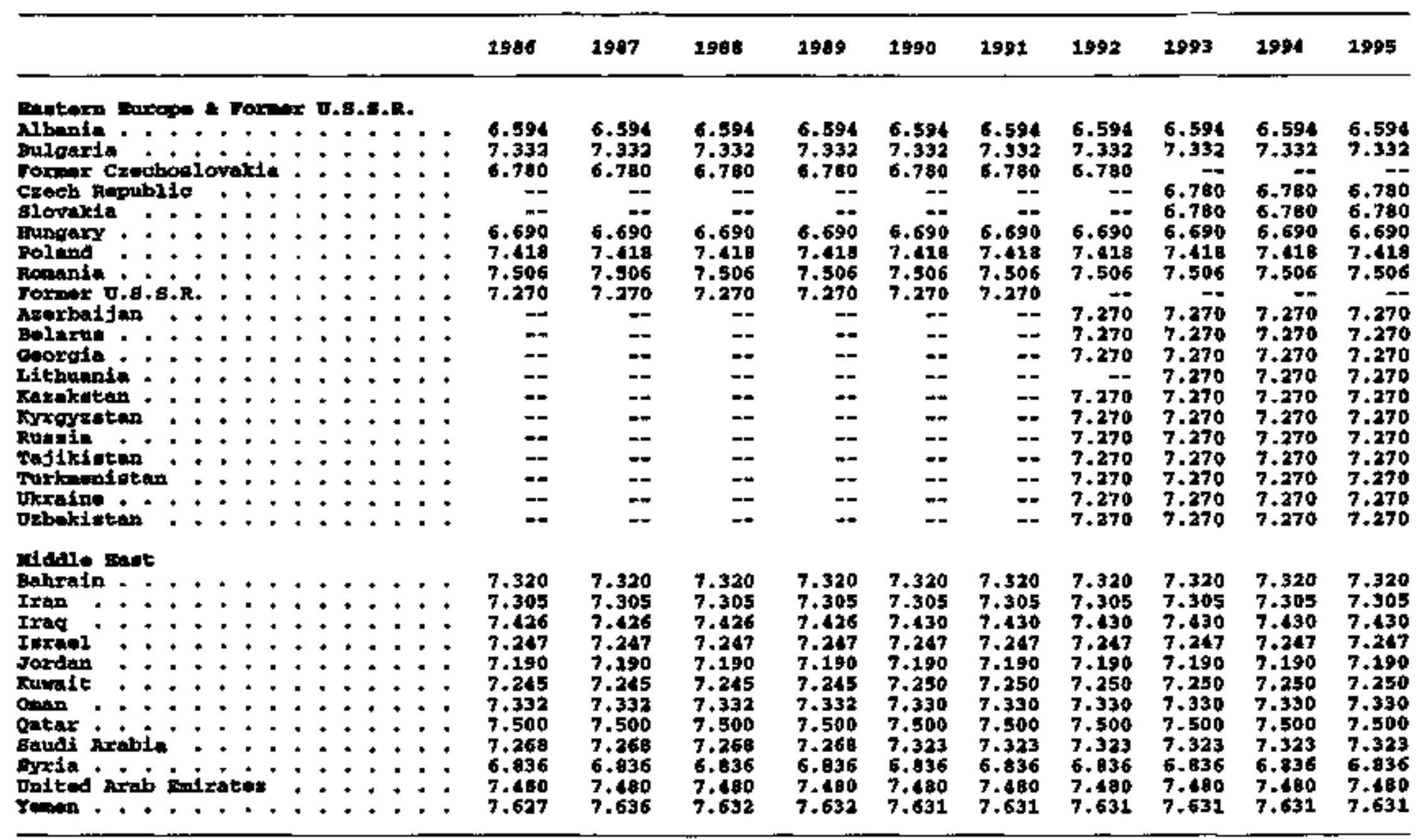

-. - Ho Production of thid form of anergy.

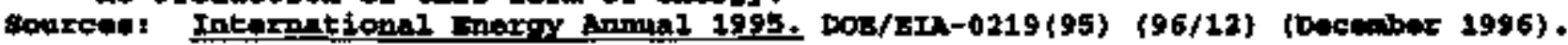


Table 22, Barrele of Cruato oil per wetric Jon, 1986-1995 (contimua)

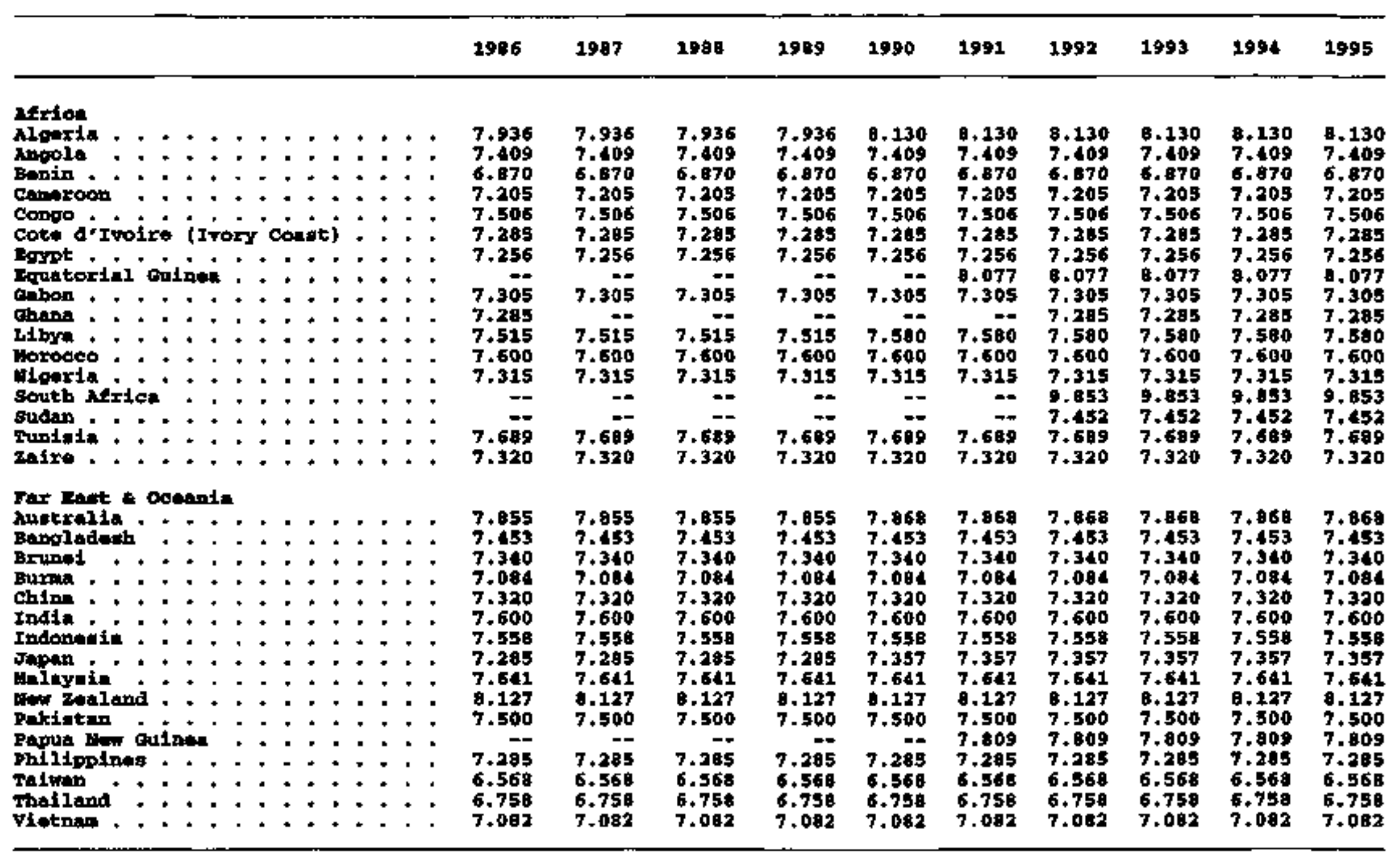

- I to Prodnction of thit torn of energy.

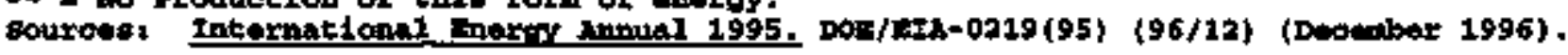

